\title{
Non-trivial higher Massey products in moment-angle complexes
}

\author{
Jelena Grbić \\ School of Mathematical Sciences, University of Southampton, UK \\ Abigail Linton \\ School of Mathematical Sciences, University of Southampton, UK
}

\begin{abstract}
As part of various obstruction theories, non-trivial Massey products have been studied in symplectic and complex geometry, commutative algebra and topology for a long time. We introduce a general approach to constructing nontrivial Massey products in the cohomology of moment-angle complexes, using homotopy theoretical and combinatorial methods. Our approach sets a unifying way of constructing higher Massey products of arbitrary cohomological classes and generalises all existing examples of non-trivial Massey products in momentangle complexes. As a result, we obtain explicit constructions of infinitely many non-formal manifolds that appear in topology, complex geometry and algebraic geometry.
\end{abstract}

\section{Introduction}

A moment-angle complex $\mathcal{Z}_{\mathcal{K}}$ over a simplicial complex $\mathcal{K}$ on $m$ vertices is built from ordered products of discs and circles in $\mathbb{C}^{m}$ that are glued together along the face category of $\mathcal{K}$. The coordinate $T^{m}$-action on $\mathbb{C}^{m}$ descends to a natural $T^{m}$ action on moment-angle complexes. If $\mathcal{K}$ is a triangulation of a sphere, the moment-angle complex $\mathcal{Z}_{\mathcal{K}}$ is a manifold that admits a smooth complex structure. These manifolds also generalise many well-known smooth complex manifolds such as Hopf and Calabi-Eckmann manifolds.

Massey products are higher operations in the homology of differential graded algebras. In the context of commutative algebra, supposing $\mathbf{k}$ is a field or $\mathbb{Z}$, the cohomology algebra of $\mathcal{Z}_{\mathcal{K}}$ is isomorphic to the Tor-algebra $\operatorname{Tor}_{\mathbf{k}[m]}(\mathbf{k}[\mathcal{K}], \mathbf{k})$ of the face ring $\mathbf{k}[\mathcal{K}]$, due to [10] and [4, Theorem 1]. The face ring $\mathbf{k}[\mathcal{K}]$ is Golod if all Massey products in $\operatorname{Tor}_{\mathbf{k}[m]}(\mathbf{k}[\mathcal{K}], \mathbf{k})$ vanish. Hence, Massey products in $\mathcal{Z}_{\mathcal{K}}$ are obstructions to Golodness of $\mathbf{k}[\mathcal{K}]$. From the perspective of complex geometry, by identifying $\mathcal{Z}_{\mathcal{K}}$ with the complement $U(\mathcal{K})$ of a coordinate subspace arrangement corresponding to $\mathcal{K}$, moment-angle complexes are LVM manifolds $[6,23]$ when $\mathcal{K}$ is the boundary of the dual of a simple polytope. 
Massey products are obstructions to the formality of these manifolds. The combinatorial approach to Massey products in moment-angle complexes has been used to prove cohomological rigidity of Löbell manifolds [8], which are built from 3-dimensional polytopes in the Pogorelov class. However, currently, most known examples of Massey products in moment-angle complexes are sporadic due to how difficult they are to calculate explicitly.

The first non-trivial Massey products in moment-angle complexes were discovered by Baskakov [5], who constructed an infinite family of triple Massey products. Limonchenko [18] constructed the first family of non-trivial $n$-Massey products for $n \geqslant 2$ on lowest-degree classes in moment-angle complexes. Families of non-trivial Massey products in moment-angle complexes associated to special geometric direct families of 2-truncated cubes (flag nestohedra) are due to Buchstaber and Limonchenko [9], who also applied these families to the differentials in Eilenberg-Moore and Milnor spectral sequences. In [20], Limonchenko constructs non-trivial higher Massey products in highly-connected moment-angle complexes by using the simplicial multiwedge operation (or Jconstruction), which takes a simplicial complex and builds a new one that has the same combinatorial structure as the original.

Using combinatorics and homotopy theory, we give the first systematic and unifying approach for constructing non-trivial Massey products in the cohomology of moment-angle complexes. We show that the combinatorics of $\mathcal{K}$ encodes Massey products. By doing this, we expose some of the structural behaviour of Massey products with respect to combinatorial operations, and spark the ability to construct concrete examples of non-trivial Massey products in commutative algebra, complex geometry and combinatorics, as well as toric topology.

Our starting point is the cup product, which is a 2-Massey product. The categorical product of simplicial complexes is the join, which is mirrored by the product of moment-angle complexes $\mathcal{Z}_{\mathcal{K}_{1} * \mathcal{K}_{2}}=\mathcal{Z}_{\mathcal{K}_{1}} \times \mathcal{Z}_{\mathcal{K}_{2}}$ and the existence of a non-trivial cup product in the cohomology of $\mathcal{Z}_{\mathcal{K}_{1} * \mathcal{K}_{2}}$. Unlike cup products, Massey products are higher operations so certain $(n-1)$-Massey products must be trivial in order to define $n$-Massey products.

There is a classification result for 3-Massey products of cohomological classes in lowest degree in moment-angle complexes [12, Theorem 6.1.1], [15], but it vitally relies on the fact that the lowest degree classes are represented combinatorially by cycles in the 1 -skeleton of $\mathcal{K}$. This technique does not generalise to higher dimensions since it is unknown how to combinatorially realise an arbitrary $n$-cycle. So far there has not been a systematic way to construct triple Massey, or any $n$-Massey, products of higher dimensional classes. We give two constructions that address these drawbacks.

In Construction 3.5, to construct non-trivial $n$-Massey products in momentangle complexes, we start with the join of $n$ simplicial complexes $\mathcal{K}_{i}$. To trivialise the lower Massey products, we systematically remove certain simplices from the join by an operation called star deletion and call the constructed simplicial complex $\mathcal{K}$. We show that $\mathcal{Z}_{\mathcal{K}}$ has a non-trivial $n$-Massey product in Theorem 3.17. It is important to emphasise that we do not impose any restrictions on $n$-arity of these Massey products, on the choice of simplicial complexes $\mathcal{K}_{i}$ for any $i$, nor 
on the dimension of classes in the Massey product. This construction generalises Baskakov's [5] family of non-trivial triple Massey products in the cohomology of moment-angle complexes, taking triangulations of spheres for $\mathcal{K}_{1}, \mathcal{K}_{2}$ and $\mathcal{K}_{3}$. Also it generalises Limonchenko's [18, Theorem 2] family of $n$-Massey products, which are built by removing simplices from the join of $n 0$-spheres.

Notably, our construction produces the first examples of non-trivial Massey products on torsion classes, as well as examples with non-trivial indeterminacy. Such an example is constructed by star deleting simplices in the join of the projective plane $\mathbb{R} P^{2}$ and two copies of the 0-sphere, as illustrated in Example 3.21. We also create the first infinite families of higher Massey products with nontrivial indeterminacy in moment-angle complexes, on arbitrary cohomological classes, by extending our construction in Section 3.2.

The topological properties and homotopy type of $\mathcal{K}$ do not determine the topology of the moment-angle complex $\mathcal{Z}_{\mathcal{K}}$. However, unexpectedly, in Construction 4.6 we deform $\mathcal{K}$ up to homotopy to create a new simplicial complex $\mathcal{L}$ such that $\mathcal{Z}_{\mathcal{L}}$ has an explicitly constructed $n$-Massey product if $\mathcal{Z}_{\mathcal{K}}$ has an $n$-Massey product. Crucially, the Massey product in $\mathcal{Z}_{\mathcal{L}}$ can be of different dimensional cohomological classes to those in $\mathcal{Z}_{\mathcal{K}}$. In this construction, the simplicial complex $\mathcal{L}$ has the same homotopy type as $\mathcal{K}$ and is obtained by systematically "stretching" certain simplices of $\mathcal{K}$. In Theorem 4.12 , we show that these Massey products are non-trivial, even if they have non-trivial indeterminacy.

Consequently, we can construct infinite families of non-trivial Massey products from known examples by "stretching" simplices in a controlled way. For example, from each of the obstruction graphs in the classification of lowest-degree triple Massey products in moment-angle complexes [12, 15], we obtain infinite families of non-trivial triple Massey products of higher dimensional classes. We give an alternative proof of known examples of non-trivial triple Massey products in moment-angle manifolds, such as those associated with Pogorelov polytopes [26] and permutahedra or stellohedra [19, 20] using "stretched" obstruction graphs. Also, the two constructions, Constructions 3.5 and 4.6, can be combined to create new higher Massey products. We use this to create $k$-Massey products in moment-angle manifolds associated with $n$-dimensional permutahedra and stellohedra for every $k<n$, including Massey products with non-trivial indeterminacy.

Even though it has been known for decades that Massey products are important obstructions in many fields, we have the first general methods to calculate and construct $n$-Massey products of classes in any degree, for any $n$, including Massey products with non-trivial indeterminacy. The first infinite family of examples of non-formal spaces or non-Golod face rings were constructed by Limonchenko [20, Theorem 4.10] using moment-angle complexes associated to graph associahedra. There are other explicit families constructed in [18], [20] and [9]. More generally, our framework constructs infinitely many families of such examples, confirming that non-trivial higher Massey products are much more common in moment-angle complexes and moment-angle manifolds than previously thought. 
Furthermore, these techniques do not just apply to moment-angle complexes. We study Massey products in moment-angle complexes via combinatorics; one key fact to do this is that the cohomology of $\mathcal{Z}_{\mathcal{K}}$ decomposes into a direct sum of cohomology groups of full subcomplexes of $\mathcal{K}[4$, Theorem 1]. For a topological pair $(X, A)$, a polyhedral product $(X, A)^{\mathcal{K}}$ is a generalisation of a moment-angle complex since $\mathcal{Z}_{\mathcal{K}}=\left(D^{2}, S^{1}\right)^{\mathcal{K}}$. In the case of a topological space $A$ and its cone $C A$, Bahri, Bendersky, Cohen and Gitler [2, Theorem 1.12] showed that the cohomology of $(C A, A)^{\mathcal{K}}$ also decomposes in terms of $H^{*}(A)$ and the cohomology of full subcomplexes of $\mathcal{K}$ when $H^{*}(A)$ satisfies the strong Künneth formula. Using this decomposition and our constructions, it is possible to produce non-trivial Massey products in $(C A, A)^{\mathcal{K}}$ by incorporating cohomological classes of $A$ to the classes we construct in the cohomology of full subcomplexes of $\mathcal{K}$ in order to create Massey products in $\mathcal{Z}_{\mathcal{K}}$.

\section{Preliminaries}

\subsection{Moment-angle complexes}

Let $\mathcal{K}$ be a simplicial complex on the vertex set $[m]=\{1, \ldots, m\}$. The moment-angle complex $\mathcal{Z}_{\mathcal{K}}$ [7, Definition 3.2.1] is

$$
\mathcal{Z}_{\mathcal{K}}=\bigcup_{\sigma \in \mathcal{K}}\left(D^{2}, S^{1}\right)^{\sigma} \subset\left(D^{2}\right)^{m}
$$

where $\left(D^{2}, S^{1}\right)^{\sigma}=\prod_{i=1}^{m} Y_{i}$ for $Y_{i}=D^{2}$ if $i \in \sigma$, and $Y_{i}=S^{1}$ if $i \notin \sigma$. A moment-angle complex $\mathcal{Z}_{\mathcal{K}}$ is a manifold if $\mathcal{K}$ is a triangulation of a sphere.

In this paper, all coefficients are in $\mathbf{k}$, which is a field or $\mathbb{Z}$. As a subspace of the polydisc, $\mathcal{Z}_{\mathcal{K}}$ has a cellular decomposition that induces a multigrading on the cellular cochain groups $C^{*}\left(\mathcal{Z}_{\mathcal{K}}\right)$. For $J \subset[m]$, the full subcomplex $\mathcal{K}_{J}$ is $\{\sigma \in \mathcal{K} \mid \sigma \subset J\}$. Let $\widetilde{C}^{*}\left(\mathcal{K}_{J}\right)$ be the augmented simplicial cochain complex. The cohomology ring of $\mathcal{Z}_{\mathcal{K}}$ can be expressed in combinatorial terms.

Theorem 2.1. [4] There is an isomorphism of cochains

$$
\widetilde{C}^{*-1}\left(\mathcal{K}_{J}\right) \rightarrow C^{*-|J|, 2 J}\left(\mathcal{Z}_{\mathcal{K}}\right) \subset C^{*+|J|}\left(\mathcal{Z}_{\mathcal{K}}\right)
$$

that induces an isomorphism of algebras

$$
H^{*}\left(\mathcal{Z}_{\mathcal{K}}\right) \cong \bigoplus_{J \subset[m]} \widetilde{H}^{*}\left(\mathcal{K}_{J}\right)
$$

where $\widetilde{H}^{-1}\left(\mathcal{K}_{\varnothing}\right)=\mathbf{k}$.

We refer to the cohomology decomposition (2.1) as Hochster's formula [16]. Let $C_{p}\left(K_{J}\right)$ be simplicial chain complex for $\mathcal{K}_{J}$. The cochain group $C^{p}\left(\mathcal{K}_{J}\right)=$ $\operatorname{Hom}\left(C_{p}\left(\mathcal{K}_{J}\right), \mathbf{k}\right)$ has a basis of $\chi_{L}$ for a $p$-simplex $L \in \mathcal{K}_{J}$, where $\chi_{L}$ takes the 
value 1 on $L$ and 0 otherwise. A subset $J \subset[m]$ has an order inherited from $[m]$. If $j$ is the $r$ th element of $J$, define

$$
\varepsilon(j, J)=(-1)^{r-1}
$$

and for $L \subset J$, define $\varepsilon(L, J)=\prod_{j \in L} \varepsilon(j, J)$. For simplices $L=\left\{l_{1}, \ldots, l_{p}\right\}$, $M=\left\{m_{1}, \ldots, m_{q}\right\}$, we denote $\left\{l_{1}, \ldots, l_{p}, m_{1}, \ldots, m_{q}\right\}$ by $L \cup M$. The product on $\bigoplus_{J \subset[m]} \widetilde{H}^{*}\left(\mathcal{K}_{J}\right)$ is induced by $C^{p-1}\left(\mathcal{K}_{I}\right) \otimes C^{q-1}\left(\mathcal{K}_{J}\right) \rightarrow C^{p+q-1}\left(\mathcal{K}_{I \cup J}\right)$,

$$
\chi_{L} \otimes \chi_{M} \mapsto \begin{cases}c_{L \cup M} \chi_{L \cup M} & \text { if } I \cap J=\varnothing, \\ 0 & \text { otherwise }\end{cases}
$$

where $c_{L \cup M}=\varepsilon(L, I) \varepsilon(M, J) \zeta \varepsilon(L \cup M, I \cup J)$ and $\zeta=\prod_{k \in I \backslash L} \varepsilon(k, k \cup J \backslash M)$.

For a cochain $a \in C^{p}\left(\mathcal{K}_{J}\right)$, let the support of $a$ be the set $S_{a}$ of $p$-simplices $\sigma \in \mathcal{K}_{J}$ such that $a=\sum_{\sigma \in S_{a}} a_{\sigma} \chi_{\sigma}$ for a nontrivial coefficient $a_{\sigma} \in \mathbf{k}$. For a cohomology class $\alpha \in \widetilde{H}^{p}\left(\mathcal{K}_{J}\right)$, we say that $\alpha$ is supported on $\mathcal{K}_{J}$.

Lemma 2.2. For a simplicial complex $\mathcal{K}$, let $a \in C^{p}\left(\mathcal{K}_{I}\right)$ and $b \in C^{q}\left(\mathcal{K}_{J}\right)$. Let the order of vertices in $\mathcal{K}$ be such that $i<j$ for every $i \in I$ and $j \in J$. Suppose that $a=\sum_{\sigma \in S_{a}} a_{\sigma} \chi_{\sigma}$ and $b=\sum_{\tau \in S_{b}} b_{\tau} \chi_{\tau}$ for $p$-simplices $\sigma \in S_{a} \subset$ $\mathcal{K}_{I}$, q-simplices $\tau \in S_{b} \subset \mathcal{K}_{J}$ and coefficients $a_{\sigma}, b_{\tau} \in \mathbf{k}$. Then the product $a b \in C^{p+q+1}\left(\mathcal{K}_{I \cup J}\right)$ is given by

$$
a b=(-1)^{|I|(q+1)} \sum_{\sigma \in S_{a}} \sum_{\tau \in S_{b}} a_{\sigma} b_{\tau} \chi_{\sigma \cup \tau} .
$$

Proof. The product $a b$ is given by

$$
\begin{aligned}
a b & =\left(\sum_{\sigma \in S_{a}} a_{\sigma} \chi_{\sigma}\right)\left(\sum_{\tau \in S_{b}} b_{\tau} \chi_{\tau}\right) \\
& =\sum_{\sigma \in S_{a}} \sum_{\tau \in S_{b}} a_{\sigma} b_{\tau} \varepsilon(\sigma, I) \varepsilon(\tau, J) \zeta \varepsilon(\sigma \cup \tau, I \cup J) \chi_{\sigma \cup \tau}
\end{aligned}
$$

where $\zeta=1$ since all vertices of $I$ are ordered before vertices of $J$ in $\mathcal{K}$.

By the definition of $\varepsilon$, and since all elements $I$ are ordered before $J, \varepsilon(\sigma \cup \tau, I \cup$ $J)=\varepsilon(\sigma, I) \varepsilon(\tau, I \cup J)$. Furthermore, for each $q$-simplex $\tau=\left\{i_{1}, \ldots, i_{q+1}\right\} \subset J$,

$$
\begin{aligned}
\varepsilon(\tau, I \cup J) & =\prod_{j \in\{1, \ldots, q+1\}} \varepsilon\left(i_{j}, I \cup J\right)=\prod_{j \in\{1, \ldots, q+1\}}(-1)^{|I|} \varepsilon\left(i_{j}, J\right) \\
& =(-1)^{|I|(q+1)} \varepsilon(\tau, J) .
\end{aligned}
$$

Therefore, since $\varepsilon(I, J)^{2}=1$ for any sets $I, J$, the statement follows.

\subsection{Massey products}

Massey products are higher cohomology operations that were introduced in a short note by Massey [21] and were thereafter first used by Massey and Uehara in [25] to prove that Whitehead products satisfy the Jacobi identity. They have many applications for example as topological invariants, obstructions to formality and for calculating differentials in spectral sequences. 
Definition 2.3. Let $(A, d)$ be a differential graded algebra with classes $\alpha_{i}$ in $H^{p_{i}}(A, d)$ for $1 \leqslant i \leqslant n$. Let $a_{i, i} \in A^{p_{i}}$ be a representative for $\alpha_{i}$. A defining system associated to $\left\langle\alpha_{1}, \ldots, \alpha_{n}\right\rangle$ is a set of elements $\left(a_{i, k}\right)$ for $1 \leqslant i \leqslant k \leqslant n$ and $(i, k) \neq(1, n)$ such that $a_{i, k} \in A^{p_{i}+\cdots+p_{k}-k+i}$ and

$$
d\left(a_{i, k}\right)=\sum_{r=i}^{k-1} \overline{a_{i, r}} a_{r+1, k}
$$

where $\overline{a_{i, r}}=(-1)^{1+\operatorname{deg} a_{i, r}} a_{i, r}$. To each defining system of $\left\langle\alpha_{1}, \ldots, \alpha_{n}\right\rangle$, the associated cocycle is defined as

$$
\sum_{r=1}^{n-1} \overline{a_{1, r}} a_{r+1, n} \in A^{p_{1}+\cdots+p_{n}-n+2} .
$$

The $n$-Massey product $\left\langle\alpha_{1}, \ldots, \alpha_{n}\right\rangle$ is the set of cohomology classes of associated cocycles for all possible defining systems. The indeterminacy of a Massey product is the set of differences between elements in $\left\langle\alpha_{1}, \ldots, \alpha_{n}\right\rangle$. The Massey product is called trivial if $0 \in\left\langle\alpha_{1}, \ldots, \alpha_{n}\right\rangle$.

We use Theorem 2.1 to give a correspondence between defining systems in $C^{*}\left(\mathcal{Z}_{\mathcal{K}}\right)$ and in $\underset{J \subset[m]}{\bigoplus} C^{*}\left(\mathcal{K}_{J}\right)$. For any $a \in C^{p+|J|+1}\left(\mathcal{Z}_{\mathcal{K}}\right)$ with $p \geqslant 0$ and $J \subset[m]$, there is a corresponding $a \in C^{p}\left(\mathcal{K}_{J}\right)$.

Definition 2.4. For $a \in C^{p}\left(\mathcal{K}_{J}\right)$, let $\overline{\operatorname{deg}}(a)=p+|J|+1$ and let $\overline{\bar{a}}=$ $(-1)^{1+\overline{\operatorname{deg}} a} a=(-1)^{p+|J|} a$.

Let $\left\langle\alpha_{1}, \ldots, \alpha_{n}\right\rangle \subset H^{*}\left(\mathcal{Z}_{\mathcal{K}}\right)$, where each class $\alpha_{i} \in H^{p_{i}+\left|J_{i}\right|+1}\left(\mathcal{Z}_{\mathcal{K}}\right)$ corresponds to $\alpha_{i} \in H^{p_{i}}\left(\mathcal{K}_{J_{i}}\right)$. Let $\left(a_{i, k}\right) \subset C^{*}\left(\mathcal{Z}_{\mathcal{K}}\right)$ be a defining system for $\left\langle\alpha_{1}, \ldots, \alpha_{n}\right\rangle$, where $a_{i, i}=a_{i}$ is a cocycle representative for $\alpha_{i}$. Then $a_{i, k} \in$ $C^{p_{i}+\cdots+p_{k}+\left|J_{i} \cup \cdots \cup J_{k}\right|+1}\left(\mathcal{Z}_{\mathcal{K}}\right)$ and $d\left(a_{i, k}\right)=\sum_{r=i}^{k-1} \overline{a_{i, r}} a_{r+1, k}$. By Theorem 2.1, there are corresponding cochains $a_{i, k} \in C^{p_{i}+\cdots+p_{k}}\left(\mathcal{K}_{J_{i} \cup \cdots \cup J_{k}}\right)$ and

$$
\begin{aligned}
\overline{\operatorname{deg}}\left(a_{i, k}\right) & =p_{i}+\cdots+p_{k}+\left|J_{i} \cup \cdots \cup J_{k}\right|+1 \\
& =\left(p_{i}+\left|J_{i}\right|+1\right)+\cdots+\left(p_{k}+\left|J_{k}\right|+1\right)-k+i \\
& =\overline{\operatorname{deg}}\left(a_{i}\right)+\cdots+\overline{\operatorname{deg}}\left(a_{k}\right)-k+i .
\end{aligned}
$$

By the product in $(2.3), d\left(a_{i, k}\right)=\sum_{r=i}^{k-1} \overline{\overline{a_{i, r}}} a_{r+1, k}$. Hence $\left(a_{i, k}\right) \subset \underset{J \subset[m]}{\bigoplus} C^{*}\left(\mathcal{K}_{J}\right)$ is a defining system that corresponds to the defining system $\left(a_{i, k}\right) \subset C^{*}\left(\mathcal{Z}_{\mathcal{K}}\right)$ and the associated cocycle $\omega \in C^{p_{1}+\cdots+p_{n}+\left|J_{1} \cup \cdots \cup J_{n}\right|+2}\left(\mathcal{Z}_{\mathcal{K}}\right)$ corresponds to the associated cocycle $\omega \in C^{p_{1}+\cdots+p_{n}+1}\left(\mathcal{K}_{J_{1} \cup \cdots \cup J_{n}}\right)$.

Let $\left\langle\alpha_{1}, \alpha_{2}, \alpha_{3}\right\rangle$ be a triple Massey product on $\alpha_{i} \in H^{p_{i}+\left|J_{i}\right|+1}\left(\mathcal{Z}_{\mathcal{K}}\right)$ for $i=1,2,3$. The indeterminacy of a triple Massey product is

$$
\alpha_{1} \cdot H^{p_{2}+p_{3}+\left|J_{2} \cup J_{3}\right|+1}\left(\mathcal{Z}_{\mathcal{K}}\right)+\alpha_{3} \cdot H^{p_{1}+p_{2}+\left|J_{1} \cup J_{2}\right|+1}\left(\mathcal{Z}_{\mathcal{K}}\right) .
$$


By Theorem 2.1, $\alpha_{i}$ corresponds to $\alpha_{i} \in \widetilde{H}^{p_{i}}\left(\mathcal{K}_{J_{i}}\right)$ and the indeterminacy of $\left\langle\alpha_{1}, \alpha_{2}, \alpha_{3}\right\rangle$ is

$$
\alpha_{1} \cdot \widetilde{H}^{p_{2}+p_{3}}\left(\mathcal{K}_{J_{2} \cup J_{3}}\right)+\alpha_{3} \cdot \widetilde{H}^{p_{1}+p_{2}}\left(\mathcal{K}_{J_{1} \cup J_{2}}\right) .
$$

In general, the indeterminacy of an $n$-Massey product can be expressed in terms of matric Massey products [22, Proposition 2.3], but this is not a helpful expression for calculations.

Example 2.5. Let $\mathcal{K}$ be the simplicial complex in Figure 1. Let $\alpha_{1}, \alpha_{2}, \alpha_{3} \in$ $H^{3}\left(\mathcal{Z}_{\mathcal{K}}\right)$ correspond to $\alpha_{1}=\left[\chi_{1}\right] \in \widetilde{H}^{0}\left(\mathcal{K}_{12}\right), \alpha_{2}=\left[\chi_{3}\right] \in \widetilde{H}^{0}\left(\mathcal{K}_{34}\right), \alpha_{3}=\left[\chi_{5}\right] \in$ $\widetilde{H}^{0}\left(\mathcal{K}_{56}\right)$. Since $\widetilde{H}^{1}\left(\mathcal{K}_{1234}\right)$ and $\widetilde{H}^{1}\left(\mathcal{K}_{3456}\right)=0$, the products $\alpha_{1} \alpha_{2} \in \widetilde{H}^{1}\left(\mathcal{K}_{1234}\right)$ and $\alpha_{2} \alpha_{3} \in \widetilde{H}^{1}\left(\mathcal{K}_{3456}\right)$ are zero.

A cochain $a_{12} \in C^{0}\left(\mathcal{K}_{1234}\right)$ such that $d\left(a_{12}\right)=\overline{\overline{\chi_{1}}} \chi_{3}=0$ is of the form $a_{12}=c_{1} \chi_{3}+c_{2}\left(\chi_{1}+\chi_{4}+\chi_{2}\right)$, for any $c_{1}, c_{2} \in \mathbf{k}$. A cochain $a_{23} \in C^{0}\left(\mathcal{K}_{3456}\right)$ such that $d\left(a_{23}\right)=\overline{\overline{\chi_{3}}} \cdot \chi_{5}=\chi_{35}$ is of the form $a_{23}=c_{3}\left(\chi_{4}+\chi_{6}+\chi_{3}+\chi_{5}\right)+\chi_{5}$ for any $c_{3} \in \mathbf{k}$. Then the associated cocycle $\omega \in C^{1}(\mathcal{K})$ is $\overline{\bar{a}}_{1} a_{23}+\overline{\bar{a}}_{12} a_{3}=$ $c_{3}\left(\chi_{14}+\chi_{16}+\chi_{15}\right)+\chi_{15}+c_{1} \chi_{35}+c_{2}\left(\chi_{15}+\chi_{25}\right)$. For $\chi_{1}, \chi_{5} \in C^{0}(\mathcal{K}), \omega=$ $c_{3} d\left(\chi_{1}\right)+\chi_{15}+\left(c_{1}-c_{2}\right) \chi_{35}+c_{2} d\left(\chi_{5}\right)$. Also, $[\omega]=\left[\chi_{15}+\left(c_{1}-c_{2}\right) \chi_{35}\right] \neq 0$ for any $c_{1}, c_{2}, c_{3} \in \mathbf{k}$. Therefore $\left\langle\alpha_{1}, \alpha_{2}, \alpha_{3}\right\rangle \subset H^{8}\left(\mathcal{Z}_{\mathcal{K}}\right)$ is non-trivial with non-trivial indeterminacy, $\alpha_{1} \cdot \widetilde{H}^{0}\left(\mathcal{K}_{3456}\right)+\alpha_{3} \cdot \widetilde{H}^{0}\left(\mathcal{K}_{1234}\right)=\alpha_{3} \cdot \widetilde{H}^{0}\left(\mathcal{K}_{1234}\right)$.

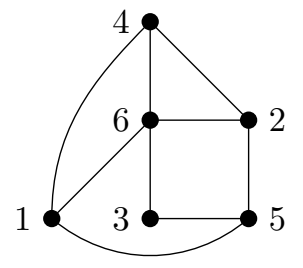

Figure 1: A simplicial complex $\mathcal{K}$ for which $\mathcal{Z}_{\mathcal{K}}$ has a non-trivial 3-Massey product with non-trivial indeterminacy.

\section{Massey products via join and star deletion}

The categorical product of simplicial complexes $\mathcal{K}_{1}$ and $\mathcal{K}_{2}$ is the join $\mathcal{K}_{1} * \mathcal{K}_{2}$. This induces a product in moment-angle complexes, $\mathcal{Z}_{\mathcal{K}_{1} * \mathcal{K}_{2}}=\mathcal{Z}_{\mathcal{K}_{1}} \times \mathcal{Z}_{\mathcal{K}_{2}}$. In this way cup products in $H^{*}\left(\mathcal{Z}_{\mathcal{K}_{1} * \mathcal{K}_{2}}\right)$ can be seen combinatorially. Since Massey products are higher operations, we require lower Massey products to be trivial. The idea is to start with the join of simplicial complexes and remove certain simplices in order to trivialise lower Massey products. To remove simplices, we use star deletion.

For a simplicial complex $\mathcal{K}$, the star and link of a simplex $I \in \mathcal{K}$ are

$$
\text { st }_{\mathcal{K}} I=\{J \in \mathcal{K} \mid I \cup J \in \mathcal{K}\} \text { and } \operatorname{lk}_{\mathcal{K}} I=\{J \in \mathcal{K} \mid I \cup J \in \mathcal{K}, I \cap J=\emptyset\} \text {. }
$$

The boundary of the star of $I \in \mathcal{K}$ is $\partial \operatorname{st}_{\mathcal{K}} I=\{J \in \mathcal{K} \mid I \cup J \in \mathcal{K}, I \not \subset J\}$. Let $\mathrm{st}_{\mathcal{K}} I=\mathrm{st}_{\mathcal{K}} I \backslash \partial \mathrm{st}_{\mathcal{K}} I$. 
Definition 3.1. The star deletion $\operatorname{sd}_{I} \mathcal{K}$ of $\mathcal{K}$ at $I$ is $\operatorname{sd}_{I} \mathcal{K}=\mathcal{K} \backslash \operatorname{st}_{\mathcal{K}} I$.

Alternatively, $\operatorname{sd}_{I} \mathcal{K}=\{J \in \mathcal{K} \mid I \not \subset J\}$. Star deletions $\operatorname{sd}_{I_{1}}$ and $\operatorname{sd}_{I_{2}}$ can be applied iteratively providing that $I_{1} \not \subset I_{2}$ and $I_{2} \not \subset I_{1}$. We show that the order of star deletions on a simplicial complex does not affect the result.

Lemma 3.2. Let $\mathcal{K}$ be a simplicial complex. Let $I_{1}, I_{2} \in \mathcal{K}$ be simplices such that $I_{1} \cap I_{2} \neq I_{1}, I_{2}$. Then $\operatorname{sd}_{I_{2}} \operatorname{sd}_{I_{1}} \mathcal{K}=\operatorname{sd}_{I_{I}} \operatorname{sd}_{I_{2}} \mathcal{K}$.

Proof. Since $I_{1} \cap I_{2} \neq I_{1}, I_{2}$, neither $I_{1} \subset I_{2}$ nor $I_{2} \subset I_{1}$. Thus $I_{1} \in \operatorname{sd}_{I_{2}} \mathcal{K}$ and

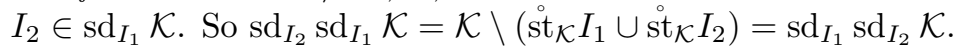

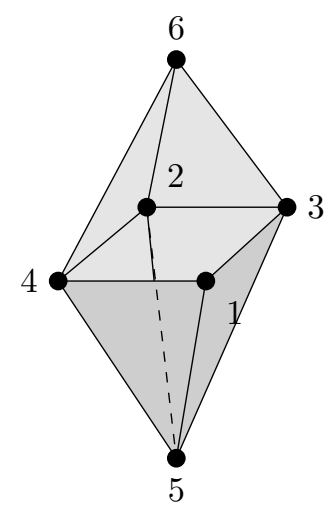

(a) $\operatorname{sd}_{\{1,6\}} \mathcal{K}$

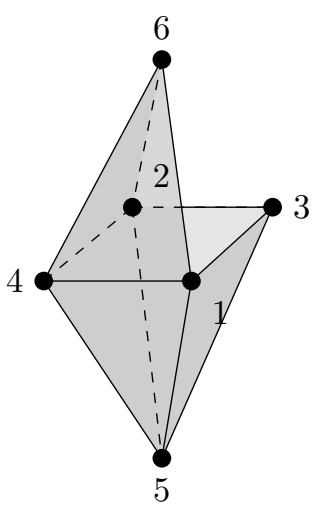

(b) $\operatorname{sd}_{\{3,6\}} \mathcal{K}$

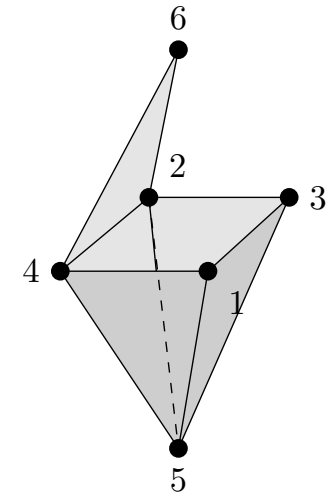

(c) $\operatorname{sd}_{\{3,6\}} \operatorname{sd}_{\{1,6\}} \mathcal{K}$

Figure 2: The star deleted complex is not affected by the order of star deletions.

Example 3.3. Let $\mathcal{K}$ be the boundary of an octahedron with opposing vertices labelled $i, i+1$ for $i=1,3,5$. Let $I_{1}=\{1,6\}$ and $I_{2}=\{3,6\}$. The star st $I_{\mathcal{K}}$ contains maximal simplices $\{1,4,6\}$ and $\{1,3,6\}$, and st $I_{\mathcal{K}} I_{2}$ contains $\{1,3,6\}$ and $\{2,3,6\}$. If the star of $I_{1}$ is deleted from $\mathcal{K}$ first, then $\operatorname{st}_{\operatorname{sd}_{I_{1}}} \mathcal{K} I_{2}$ contains the maximal simplex $\{2,3,6\}$. Hence $\operatorname{sd}_{I_{2}} \operatorname{sd}_{I_{1}} \mathcal{K}$ removes the simplices $\{1,4,6\},\{1,3,6\}$ and $\{2,3,6\}$ from $\mathcal{K}$. The same simplices are removed from $\mathcal{K}$ in $\operatorname{sd}_{I_{1}} \operatorname{sd}_{I_{2}} \mathcal{K}$, as shown in Figure 2 .

Remark 3.4. Star deletion is equivalent to doing a stellar subdivision $\operatorname{ss}_{I} \mathcal{K}=$ $\left(\mathcal{K} \backslash \mathrm{st}_{\mathcal{K}} I\right) \cup_{\partial \mathrm{st}_{\mathcal{K}} I}$ cone $\left(\partial \mathrm{st}_{\mathcal{K}} I\right)$ then restricting to the original vertices $V(\mathcal{K})$. For example, see Figure 3 compared to Figure 2a. If $\mathcal{K}$ is a triangulation of an $n$-sphere on $m$ vertices, then $\mathcal{Z}_{\mathcal{K}}$ is an $(m+n+1)$-dimensional manifold. As $\mathrm{ss}_{I} \mathcal{K} \simeq \mathcal{K}, \mathrm{ss}_{I} \mathcal{K}$ is a triangulation of an $n$-sphere on $m+1$ vertices. Hence $\mathcal{Z}_{\mathrm{ss}_{I} \mathcal{K}}$ is an $(m+n+2)$-dimensional manifold. Since Massey products are obstructions to formality, a non-trivial Massey product in $H^{*}\left(\mathcal{Z}_{\mathrm{ss}_{I}} \mathcal{K}\right)$ implies that $\mathcal{Z}_{\mathrm{ss}_{I}} \mathcal{K}$ is a non-formal. 


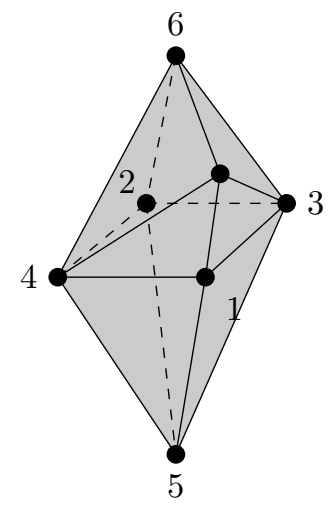

Figure 3: A stellar subdivision at $\{1,6\}$ in the octahedron

\subsection{A construction of non-trivial Massey products}

We aim to construct a simplicial complex $\mathcal{K}$ such that there is a non-trivial $n$-Massey product in $H^{*}\left(\mathcal{Z}_{\mathcal{K}}\right)$. We start with the join of $n$-simplicial complexes $\mathcal{K}^{1} * \cdots * \mathcal{K}^{n}$ and classes $\alpha_{i} \in \widetilde{H}^{p_{i}}\left(\mathcal{K}_{J_{i}}^{i}\right)$ for each $i \in\{1, \ldots, n\}$. In $\mathcal{K}^{1} * \cdots * \mathcal{K}^{n}$, all cup products between $\alpha_{i}$ s are non-trivial, so in order to define a higher Massey product we first remove simplices to make certain cup products trivial. To define which simplices to remove, we define two sets of simplices, $S_{a_{i}} \subset \mathcal{K}^{i}$ and $P_{a_{i}} \subset \mathcal{K}^{i}$ for each $\mathcal{K}^{i}$. In order to create $\mathcal{K}$, we star delete $\mathcal{K}^{1} * \cdots * \mathcal{K}^{n}$ at every simplex $\sigma_{i} \cup \sigma_{k}$ for $\sigma_{i} \in S_{a_{i}}$ and $\sigma_{k} \in P_{a_{k}}, 1 \leq i<k \leq n,(i, k) \neq(1, n)$. The star deletions at $\sigma_{1} \cup \sigma_{2}$ and $\sigma_{2} \cup \sigma_{3}$ trivialise the cup products $\alpha_{1} \alpha_{2}$ and $\alpha_{2} \alpha_{3}$ respectively, which is required to define a triple Massey product $\left\langle\alpha_{1}, \alpha_{2}, \alpha_{3}\right\rangle$. By star deleting at $\sigma_{1} \cup \sigma_{3}$, we trivialise $\left\langle\alpha_{1}, \alpha_{2}, \alpha_{3}\right\rangle$. If we also star delete at simplices $\sigma_{3} \cup \sigma_{4}$ and $\sigma_{2} \cup \sigma_{4}$, then $\left\langle\alpha_{2}, \alpha_{3}, \alpha_{4}\right\rangle$ is defined and trivial, so the 4 -Massey product $\left\langle\alpha_{1}, \alpha_{2}, \alpha_{3}, \alpha_{4}\right\rangle$ is defined. We define the Massey product $\left\langle\alpha_{1}, \ldots, \alpha_{n}\right\rangle \subset H^{*}\left(\mathcal{Z}_{\mathcal{K}}\right)$ by iterating this process.

Construction 3.5. For $i \in\{1, \ldots, n\}$, let $\mathcal{K}^{i}$ be a simplicial complex on $\left[m_{i}\right]$ vertices that is not an $\left(m_{i}-1\right)$-simplex. Since $\mathcal{K}^{i}$ is not a simplex, there is a nonzero cohomology class $\alpha_{i} \in \widetilde{H}^{p_{i}}\left(\mathcal{K}_{J_{i}}^{i}\right)$ for $p_{i} \in \mathbb{N}, J_{i} \subseteq\left[m_{i}\right]$. Let $a_{i} \in C^{p_{i}}\left(\mathcal{K}_{J_{i}}^{i}\right)$ be a cocycle representative for $\alpha_{i}$ that is supported on $p_{i}$-simplices $S_{a_{i}} \subset \mathcal{K}$ so that $a_{i}=\sum_{\sigma_{i} \in S_{a_{i}}} c_{\sigma_{i}} \chi_{\sigma_{i}} \in C^{p_{i}}\left(\mathcal{K}_{J_{i}}^{i}\right)$ for a non-zero coefficient $c_{\sigma_{i}} \in \mathbf{k}$. For every simplex $\sigma_{i} \in S_{a_{i}}$, let $v_{\sigma_{i}}$ denote one vertex in $\sigma_{i}$. Let $P_{\sigma_{i}}$ be the set

$$
P_{\sigma_{i}}=\left\{\sigma_{i}^{\prime} \in \mathcal{K}^{i} \mid \sigma_{i}^{\prime} \text { is a } p_{i} \text {-simplex, } \sigma_{i} \cap \sigma_{i}^{\prime}=\sigma_{i} \backslash v_{\sigma_{i}}\right\} .
$$

An example is shown in Figure 4.

We start by constructing the set $P_{a_{i}}$ for each $i$, in order to define star deletions of $\mathcal{K}^{1} * \cdots * \mathcal{K}^{n}$. We fix an order on the simplices in $S_{a_{i}}$ and define an ordered subsequence of simplices $\sigma_{i}^{(1)}, \ldots, \sigma_{i}^{(l)} \subset S_{a_{i}}$. Let $\sigma_{i}^{(1)}$ be the first element of $S_{a_{i}}$. Then let $S_{a_{i}}^{(1)}=S_{a_{i}} \backslash P_{\sigma_{i}^{(1)}}$. Let $\sigma_{i}^{(2)}$ be the next element after $\sigma_{i}^{(1)}$ 


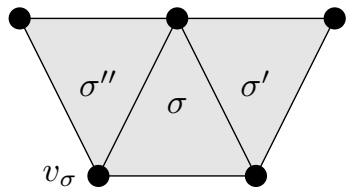

Figure 4: For this choice of vertex $v_{\sigma} \in \sigma, \sigma^{\prime} \in P_{\sigma}$ but $\sigma^{\prime \prime} \notin P_{\sigma}$

in $S_{a_{i}}^{(1)}$. Then let $S_{a_{i}}^{(2)}=S_{a_{i}}^{(1)} \backslash P_{\sigma_{i}^{(2)}}$. We continue repeatedly until $\sigma_{i}^{(l)}$ is the last element of $S_{a_{i}}^{(l-1)}$, and let

$$
P_{a_{i}}=P_{\sigma_{i}^{(1)}} \cup \cdots \cup P_{\sigma_{i}^{(l)}} .
$$

Since $\sigma_{i}^{(l)} \notin P_{\sigma_{i}^{(l)}}$, the set $S_{a_{i}} \backslash P_{a_{i}}=S_{a_{i}}^{(l-1)} \backslash P_{\sigma_{i}^{(l)}}$ contains at least the last element $\sigma_{i}^{(l)}$. So $P_{a_{i}} \neq S_{a_{i}}$.

Let $\overline{\mathcal{K}}=\mathcal{K}^{1} * \cdots * \mathcal{K}^{n}$, so $\overline{\mathcal{K}}_{\left[m_{i}\right]}=\mathcal{K}^{i}$ for every $i \in\{1, \ldots, n\}$. The vertices in each vertex set $V\left(\mathcal{K}^{i}\right)=\left[m_{i}\right]$ have an order. Suppose that the vertex set $V(\overline{\mathcal{K}})=\bigsqcup_{i \in\{1, \ldots, n\}} V\left(\mathcal{K}^{i}\right)$ is ordered so that $u<v$ for all $u \in V\left(\mathcal{K}^{i}\right)$ and $v \in V\left(\mathcal{K}^{j}\right)$ for $i<j$. We construct a simplicial complex $\mathcal{K}$ by star deleting $\overline{\mathcal{K}}$ at each simplex $\sigma_{i} \cup \sigma_{k}$ one at a time, where $\sigma_{i} \in S_{a_{i}}$ and $\sigma_{k} \in P_{a_{k}}, 1 \leq i<k \leq n$, $(i, k) \neq(1, n)$. Let $\mathcal{K}$ denote the resulting simplicial complex.

Lemma 3.6. For any $i \in\{1, \ldots, n\}$, the set $P_{a_{i}}$ is non-empty.

Proof. If $p_{i}=0$ and $\widetilde{H}^{0}(\mathcal{K}) \neq 0$, then $\mathcal{K}$ is a disjoint union of at least two vertices. For any $v, w \in \mathcal{K}, v \cap w=\emptyset=v \backslash v$. Hence $w \in P_{v}$. Alternatively let $p_{i}>0$. Since $\alpha_{i} \in \widetilde{H}^{p_{i}}\left(\mathcal{K}_{J_{i}}\right)$ is non-zero, there is a non-zero cycle $x \in C_{p_{i}}\left(\mathcal{K}_{J_{i}}\right)$ such that $a_{i}(x) \neq 0$. Let $x=\sum_{\tau \in S_{x}} c_{\tau} \Delta_{\tau}$ with non-zero coefficients $c_{\tau}$ and $p_{i^{-}}$ simplices $\tau$. Let $\sigma \in S_{a_{i}} \cap S_{x}$. Let $\partial: C_{p_{i}}\left(\mathcal{K}_{J_{i}}\right) \rightarrow C_{p_{i}-1}\left(\mathcal{K}_{J_{i}}\right)$ be the boundary homomorphism. Since $x$ is a cycle and $\partial \Delta_{\sigma} \neq 0$, for any vertex $v \in \sigma$ there exists a different simplex $\tau \in S_{x}$ with $\sigma \backslash v=\sigma \cap \tau=\tau \backslash u$ for some vertex $u \in \tau$. Hence for any $\sigma \in S_{a_{i}} \cap S_{x}, \tau \in P_{\sigma}$ and so $P_{a_{i}}$ is non-empty.

Example 3.7 (a). Let $\mathcal{K}^{1}$ be the disjoint union of two vertices $\{1\},\{2\}$ and $\mathcal{K}^{2}$ the simplicial complex in Figure 5a. The join $\mathcal{K}^{1} * \mathcal{K}^{2}$ is homotopy equivalent to $S^{2} \vee S^{1}$. Let $\alpha_{1} \in \widetilde{H}^{0}\left(\mathcal{K}^{1}\right), \alpha_{2} \in \widetilde{H}^{0}\left(\mathcal{K}^{2}\right)$ be represented by the cochains $a_{1}=\chi_{1}$ and $a_{2}=\chi_{3}+\chi_{4}+\chi_{5}$, respectively. Then $S_{a_{1}}=\{\{1\}\}$, and $S_{a_{2}}=$ $\{\{3\},\{4\},\{5\}\}$. Following the construction above, for $\sigma_{2}=\{3\}$ there is only one choice of a vertex $v=3$. Then $P_{\{3\}}=\{\{4\},\{5\},\{6\}\}$ so $S_{a_{2}}^{(1)}=\{\{3\}\}$ and $P_{a_{2}}=P_{\{3\}}$. The simplicial complex

$$
\mathcal{K}=\operatorname{sd}_{\{1,6\}} \operatorname{sd}_{\{1,5\}} \operatorname{sd}_{\{1,4\}} \mathcal{K}^{1} * \mathcal{K}^{2}
$$

is shown in Figure 5b. Since $\mathcal{K}$ is contractible, the cup product $\alpha_{1} \alpha_{2}$ is trivial.

(b). In addition to $\mathcal{K}^{1}$ and $\mathcal{K}^{2}$ in Part (a), let $\mathcal{K}^{3}$ be the disjoint union of two vertices $\{7\},\{8\}$. Let $\alpha_{3} \in \widetilde{H}^{0}\left(\mathcal{K}^{3}\right)$ be represented by $a_{3}=\chi_{7}$. Then 
$S_{a_{3}}=\{\{7\}\}$ and $P_{a_{3}}=P_{\{7\}}=\{\{8\}\}$. By Construction 3.5, we star delete $\mathcal{K}^{1} * \mathcal{K}^{2} * \mathcal{K}^{3}$ at $\sigma_{i} \cup \sigma_{k}$ for every $\sigma_{i} \in S_{a_{i}}$ and $\sigma_{k} \in P_{a_{k}}$ for $i=1,2$ and $k=i+1$. Since $S_{a_{2}}=\{\{3\},\{4\},\{5\}\}$, we obtain the simplicial complex

$$
\mathcal{K}^{\prime}=\operatorname{sd}_{\{5,8\}} \operatorname{sd}_{\{4,8\}} \operatorname{sd}_{\{3,8\}} \operatorname{sd}_{\{1,6\}} \operatorname{sd}_{\{1,5\}} \operatorname{sd}_{\{1,4\}} \mathcal{K}^{1} * \mathcal{K}^{2} * \mathcal{K}^{3} .
$$

The full subcomplex $\mathcal{K}_{3,4,5,6,7,8}^{\prime}$ is shown in Figure 5c. Theorem 3.17 will show that there is a non-trivial triple Massey product in $H^{*}\left(\mathcal{Z}_{\mathcal{K}^{\prime}}\right)$.

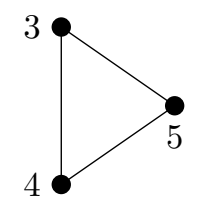

(a) The simplicial complex

60

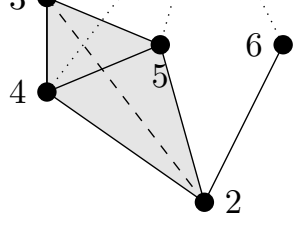

(b) $\mathcal{K}$ after star deletions at $\{1,4\},\{1,5\},\{1,6\}$

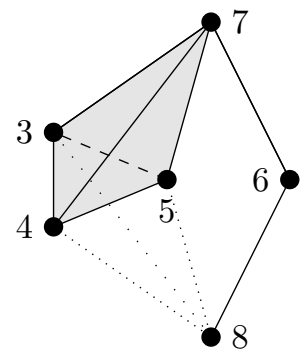

(c) $\quad \mathcal{K}_{3,4,5,6,7,8}^{\prime} \quad$ af$\{3,8\},\{4,8\},\{5,8\}$

Figure 5

Lemma 3.8. The simplicial complex $\mathcal{K}$ is independent of the order of simplices in $P_{a_{k}}$.

Proof. For any $\sigma_{k}, \sigma_{k}^{\prime} \in P_{a_{k}}$, we have $\left(\sigma_{i} \cup \sigma_{k}\right) \cap\left(\sigma_{i} \cup \sigma_{k}^{\prime}\right) \neq \sigma_{i} \cup \sigma_{k}, \sigma_{i} \cup \sigma_{k}^{\prime}$. So by Lemma 3.2, the order of $P_{a_{k}}$ does not affect $\mathcal{K}$.

Lemma 3.9. The simplicial complex $\mathcal{K}$ is independent of the order in which the pairs $\{i, k\}, 1 \leqslant i<k \leqslant n$, are chosen.

Proof. Let $\left\{i_{1}, k_{1}\right\}$ and $\left\{i_{2}, k_{2}\right\}$ be two pairs of indices. For $1 \leqslant i_{j}<k_{j} \leqslant n$, $j=1,2$, let $\sigma_{i_{j}} \in S_{a_{i_{j}}}$ and $\sigma_{k_{j}} \in P_{a_{k_{j}}}$. The intersection $\left(\sigma_{i_{1}} \cup \sigma_{k_{1}}\right) \cap\left(\sigma_{i_{2}} \cup \sigma_{k_{2}}\right)$ is empty since the vertices of any $\sigma_{j} \in S_{a_{j}}$ are a subset of $J_{j}$ for every $j \in$ $\{1, \ldots, n\}$ and $\left(J_{i_{1}} \cup J_{k_{1}}\right) \cap\left(J_{i_{2}} \cup J_{k_{2}}\right)=\emptyset$. Therefore by Lemma 3.2, we can star delete at simplices $\sigma_{i_{1}} \cup \sigma_{k_{1}}$ and simplices $\sigma_{i_{2}} \cup \sigma_{k_{2}}$ in either order.

Lemma 3.10. In Construction 3.5, the simplicial complex $\mathcal{K}$ depends on the order of simplices in $S_{a_{k}}$.

Proof. Suppose that $\sigma_{k} \in S_{a_{k}}, \sigma_{k}^{\prime} \in P_{\sigma_{k}}$ and let $\sigma_{i} \in S_{a_{i}}$ for an $i \in\{1, \ldots, k-1\}$. If $\sigma_{k}^{\prime} \in S_{a_{k}} \cap P_{\sigma_{k}}$, then either $\sigma_{k}^{\prime}>\sigma_{k}$ or $\sigma_{k}^{\prime}<\sigma_{k}$ in the order of simplices in $S_{a_{k}}$. In the first case, $\sigma_{k}^{\prime} \in P_{a_{k}}$ so we perform a star deletion at $\sigma_{i} \cup \sigma_{k}^{\prime}$. If there is no simplex $\sigma_{k}^{\prime \prime} \in S_{a_{k}}$ such that $\sigma_{k}^{\prime \prime}>\sigma_{k}$ and $\sigma_{k} \in P_{\sigma_{k}^{\prime \prime}}$, then $\sigma_{k} \notin P_{a_{k}}$. So $\sigma_{i} \cup \sigma_{k} \in \mathcal{K}$ and $\sigma_{i} \cup \sigma_{k}^{\prime} \notin \mathcal{K}$. In the second case, if the chosen vertex $v_{k^{\prime}} \in \sigma_{k}^{\prime}$ is such that $\sigma_{k}^{\prime} \backslash v_{k^{\prime}}=\sigma_{k} \backslash v_{k}$, then $\sigma_{k} \in P_{\sigma_{k}^{\prime}}$. Since $\sigma_{k}^{\prime}<\sigma_{k}, \sigma_{k} \in P_{a_{k}}$ and therefore $\sigma_{i} \cup \sigma_{k} \notin \mathcal{K}$. Hence $\mathcal{K}$ is different in the two cases. 
Lemma 3.11. The choice of vertex $v_{\sigma_{k}} \in \sigma_{k}$ affects the number of stars deletions performed in Construction 3.5.

Proof. We demonstrate this with an example. Consider the join $\mathcal{K}^{1} * \mathcal{K}^{2} * \mathcal{K}^{3}$ of three simplicial complexes. Suppose that $\mathcal{K}^{2}$ is the boundary of a tetrahedron on the vertices $1,2,3,4$. Also suppose that $a_{2} \in C^{1}\left(\mathcal{K}^{2}\right)$ is $\chi_{123}+\chi_{234}$. We fix the order on $S_{a_{2}}=\{\{1,2,3\},\{2,3,4\}\}$. First let $v_{\{123\}}=3 \in\{1,2,3\}$ and $v_{\{234\}}=$ $2 \in\{2,3,4\}$. By definition, $P_{\{123\}}=\left\{\sigma \in \mathcal{K}^{2} \mid \sigma\right.$ is a 1-simplex and $\sigma \cap$ $\left.\{1,2,3\}=\{1,2,3\} \backslash v_{\{123\}}\right\}=\{1,2,4\}$. Similarly $P_{\{234\}}=\{1,3,4\}$. Therefore $P_{a_{2}}=\{\{1,2,4\},\{1,3,4\}\}$. To construct $\mathcal{K}$ from $\mathcal{K}^{1} * \mathcal{K}^{2} * \mathcal{K}^{3}$, we perform $\left|S_{a_{1}}\right|\left|P_{a_{2}}\right|+\left|S_{a_{2}}\right|\left|P_{a_{3}}\right|=2\left|S_{a_{1}}\right|+\left|S_{a_{2}}\right|\left|P_{a_{3}}\right|$ star deletions.

Compare this to the case when $v_{\{123\}}=1$, so $P_{\{123\}}=\{2,3,4\}$. Since $\{1,2,3\}$ comes before $\{2,3,4\}$ in $S_{a_{2}}$ and $S_{a_{2}} \backslash P_{\{123\}}=\{1,2,3\}, P_{a_{2}}=P_{\{123\}}=$ $\{2,3,4\}$. In this case, to construct $\mathcal{K}$ we perform $\left|S_{a_{1}}\right|+\left|S_{a_{2}}\right|\left|P_{a_{3}}\right|$ star deletions. Since $S_{a_{1}}$ and $P_{a_{3}}$ do not depend on $v_{\{123\}}$ or $v_{\{234\}}$, this is fewer star deletions than when $v_{\{123\}}=3$.

We will prove that the Massey product $\left\langle\alpha_{1}, \ldots, \alpha_{n}\right\rangle \subset H^{*}\left(\mathcal{Z}_{\mathcal{K}}\right)$ is non-trivial in several steps, first showing that it is defined.

Proposition 3.12. Let $\mathcal{K}$ be a simplicial complex constructed in Construction 3.5. Then $\left\langle\alpha_{1}, \ldots, \alpha_{n}\right\rangle \subset H^{*}\left(\mathcal{Z}_{\mathcal{K}}\right)$ is defined.

Proof. Let $a_{i}=\sum_{\sigma_{i} \in S_{a_{i}}} c_{\sigma_{i}} \chi_{\sigma_{i}}$ be a representative cocycle for $\alpha_{i} \in \widetilde{H}^{p_{i}}\left(\mathcal{K}_{J_{i}}\right)$ for each $i \in\{1, \ldots, n-1\}$. We construct a defining system $\left(a_{i, k}\right)$ for the Massey product $\left\langle\alpha_{1}, \ldots, \alpha_{n}\right\rangle \subset H^{p_{1}+\cdots+p_{n}+\left|J_{1} \cup \cdots \cup J_{n}\right|+2}\left(\mathcal{Z}_{\mathcal{K}}\right)$.

For $1 \leqslant i \leqslant k \leqslant n,(i, k) \neq(1, n)$, let $a_{i, k} \in C^{p_{i}+\cdots+p_{k}}\left(\mathcal{K}_{J_{i} \cup \cdots \cup J_{k}}\right)$ be the cochain given by

$$
a_{i, k}=\sum_{\sigma_{i} \in S_{a_{i}}} \sum_{\sigma_{i+1} \in \widetilde{S}_{a_{i+1}}} \cdots \sum_{\sigma_{k} \in \widetilde{S}_{a_{k}}} c_{\sigma_{i}} \ldots c_{\sigma_{k}} \theta_{i, k} \chi_{\sigma_{i} \cup \cdots \cup \sigma_{k} \backslash\left(v_{i+1} \cup \cdots \cup v_{k}\right)}
$$

where $\widetilde{S}_{a_{i}}=S_{a_{i}} \backslash P_{a_{i}}$, vertices $v_{i}=v_{\sigma_{i}} \in \sigma_{i}$ are fixed, and $\theta_{i, k}=1$ when $i=k$ or otherwise

$$
\begin{aligned}
\left.\theta_{i, k}=(-1)^{k-i+\left|J_{i}\right|\left(p_{i+1}+\cdots+p_{k}\right)+\left|J_{i+1}\right|\left(p_{i+2}+\cdots\right.}+p_{k}\right)+\cdots+\left|J_{k-1}\right| p_{k} \\
\cdot \varepsilon\left(v_{i+1}, \sigma_{i+1}\right) \ldots \varepsilon\left(v_{k}, \sigma_{k}\right) .
\end{aligned}
$$

For any $\sigma_{i} \in \widetilde{S}_{a_{i}}$ and $\sigma_{k} \in \widetilde{S}_{a_{k}}, \sigma_{i} \cup \sigma_{k} \in \mathcal{K}$ and so $\sigma_{i} \cup \cdots \cup \sigma_{k} \backslash\left(v_{i+1} \cup \cdots \cup v_{k}\right) \in$ $\mathcal{K}$. Since every coefficient $c_{\sigma_{i}}$ is non-zero and each $\chi_{\sigma_{i} \cup \ldots \cup \sigma_{k} \backslash\left(v_{i+1} \cup \cdots \cup v_{k}\right)}$ is a different basis element of $C^{p_{i}+\cdots+p_{k}}\left(\mathcal{K}_{J_{i} \cup \cdots \cup J_{k}}\right)$, the cochain $a_{i, k}$ is not trivial.

We will verify that $d\left(a_{i, k}\right)=\sum_{r=i}^{k-1} \overline{\overline{a_{i, r}}} \cdot a_{r+1, k}$. By the definition of the 
coboundary map,

$$
\begin{aligned}
& d\left(a_{i, k}\right)=\sum_{\sigma_{i} \in S_{a_{i}}} \sum_{\sigma_{i+1} \in \widetilde{S}_{a_{i+1}}} \cdots \sum_{\sigma_{k} \in \widetilde{S}_{a_{k}}} c_{\sigma_{i}} \ldots c_{\sigma_{k}} \theta_{i, k} . \\
& \left(\sum_{j \in B} \varepsilon\left(j, j \cup \sigma_{i} \cup \cdots \cup \sigma_{k} \backslash\left(v_{i+1} \cup \cdots \cup v_{k}\right)\right) \chi_{j \cup \sigma_{i} \cup \cdots \cup \sigma_{k} \backslash\left(v_{i+1} \cup \cdots \cup v_{k}\right)}\right)
\end{aligned}
$$

where $B$ is the set $\left\{j \in J_{i} \cup \cdots \cup J_{k} \backslash\left(\sigma_{i} \cup \cdots \cup \sigma_{k} \backslash\left(v_{i+1} \cup \cdots \cup v_{k}\right)\right) \mid j \cup \sigma_{i} \cup\right.$ $\left.\cdots \cup \sigma_{k} \backslash\left(v_{i+1} \cup \cdots \cup v_{k}\right) \in \mathcal{K}\right\}$. First we show that the only non-zero summands are when $j \in v_{i+1} \cup \cdots \cup v_{k}$. For fixed $\sigma_{i}, \ldots, \sigma_{k}$, suppose that there is a vertex $j \in J_{i} \cup \cdots \cup J_{k} \backslash\left(\sigma_{i} \cup \cdots \cup \sigma_{k}\right)$ such that $j \cup \sigma_{i} \cup \cdots \cup \sigma_{k} \backslash\left(v_{i+1} \cup \cdots \cup v_{k}\right) \in \mathcal{K}$. So $j \notin v_{i+1} \cup \cdots \cup v_{k}$. Consider two cases, either $j \in J_{i}$ or $j \in J_{l} \backslash \sigma_{l}$ for $l \in\{i+1, \ldots, k\}$.

(i) In the first case, $j \in J_{i}$. By the definition of the coboundary map and since $a_{i}$ is a cocycle,

$$
d\left(a_{i}\right)=\sum_{\sigma \in S_{a_{i}}} c_{\sigma} \sum_{j \in J_{i} \backslash V(\sigma)} \varepsilon(j, j \cup \sigma) \chi_{j \cup \sigma}=0 .
$$

We extend this sum by taking the union of each $j \cup \sigma$ with $\sigma_{i+1} \cup \cdots \cup \sigma_{k} \backslash$ $\left(v_{i+1} \cup \cdots \cup v_{k}\right)$. Since $\sigma_{l} \notin P_{a_{l}}$ for every $l \in\{i+1, \ldots, k\}, j \cup \sigma \cup \sigma_{i+1} \cup \cdots \cup$ $\sigma_{k} \backslash\left(v_{i+1} \cup \cdots \cup v_{k}\right) \in \mathcal{K}$ for any $j \cup \sigma \in \mathcal{K}_{J_{i}}$. Hence

$$
\begin{aligned}
\sum_{\sigma \in S_{a_{i}}} c_{\sigma} \sum_{j \in J_{i} \backslash V(\sigma)} \varepsilon\left(j, j \cup \sigma \cup \sigma_{i+1} \cup \cdots \cup\right. & \left.\sigma_{k} \backslash\left(v_{i+1} \cup \cdots \cup v_{k}\right)\right) . \\
& \cdot \chi_{j \cup \sigma \cup \sigma_{i+1} \cup \cdots \cup \sigma_{k} \backslash\left(v_{i+1} \cup \cdots \cup v_{k}\right)}=0 .
\end{aligned}
$$

(ii) In the second case, $j \in J_{l}$ for $l \in\{i+1, \ldots, k\}$, so $j \cup \sigma_{l} \backslash v_{l} \in \mathcal{K}_{J_{l}}$ and hence $j \cup \sigma_{l} \backslash v_{l} \in P_{\sigma_{l}} \subset P_{a_{l}}$. By Construction 3.5, $\sigma_{i} \cup j \cup \sigma_{l} \backslash v_{l} \notin \mathcal{K}$. Hence $j \cup \sigma_{i} \cup \cdots \cup \sigma_{k} \backslash\left(v_{i+1} \cup \cdots \cup v_{k}\right) \notin \mathcal{K}$ for any $j \in J_{i} \cup \cdots \cup J_{k} \backslash\left(v_{i+1} \cup \cdots \cup v_{k}\right)$.

Since the only non-zero summands in (3.4) are when $j \in v_{i+1} \cup \cdots \cup v_{k}$, $d\left(a_{i, k}\right)$ reduces to

$$
\begin{aligned}
& d\left(a_{i, k}\right)=\sum_{\sigma_{i} \in S_{a_{i}}} \sum_{\sigma_{i+1} \in \widetilde{S}_{a_{i+1}}} \cdots \sum_{\sigma_{k} \in \widetilde{S}_{a_{k}}} c_{\sigma_{i}} \ldots c_{\sigma_{k}} \theta_{i, k} . \\
& \quad \sum_{\substack{j \in v_{i+1} \cup \cdots \cup v_{k} \mid \\
j \cup \sigma_{i} \cup \cdots \cup \sigma_{k} \backslash\left(v_{i+1} \cup \cdots \cup v_{k}\right) \in \mathcal{K}}} \varepsilon\left(j, j \cup \sigma_{i} \cup \cdots \cup \sigma_{k} \backslash\left(v_{i+1} \cup \cdots \cup v_{k}\right)\right) \chi_{j \cup \sigma_{i} \cup \cdots \cup \sigma_{k} \backslash\left(v_{i+1} \cup \cdots \cup v_{k}\right)} .
\end{aligned}
$$

Denote $j \in v_{i+1} \cup \cdots \cup v_{k}$ by $v_{r+1}$ for $r \in\{i, \ldots, k-1\}$, and rewrite $d\left(a_{i, k}\right)$ as

$$
\begin{aligned}
d\left(a_{i, k}\right)= & \sum_{r=i}^{k-1} \theta_{i, k} \sum_{\sigma_{i} \in S_{a_{i}}} \sum_{\sigma_{i+1} \in \widetilde{S}_{a_{i+1}}} \cdots \sum_{\sigma_{k} \in \widetilde{S}_{a_{k}}} c_{\sigma_{i}} \ldots c_{\sigma_{k}} . \\
& \cdot \varepsilon\left(v_{r+1}, \sigma_{i} \cup \cdots \cup \sigma_{k} \backslash\left(v_{i+1} \cup \cdots \cup \hat{v}_{r+1} \cup \cdots \cup v_{k}\right)\right) . \\
& \cdot \chi_{\sigma_{i} \cup \cdots \cup \sigma_{k} \backslash\left(v_{i+1} \cup \cdots \cup \hat{v}_{r+1} \cup \cdots \cup v_{k}\right)}
\end{aligned}
$$


where $\hat{v}_{r+1}$ denotes that $v_{r+1}$ is deleted from the sequence $v_{i+1}, \ldots, v_{k}$.

To show that $d\left(a_{i, k}\right)=\sum_{r=i}^{k-1} \overline{\overline{a_{i, r}}} \cdot a_{(r+1), k}$, we write out $a_{i, r}$ and $a_{(r+1), k}$ so that $\sum_{r=i}^{k-1} \overline{\overline{a_{i, r}}} \cdot a_{(r+1), k}$ is

$$
\begin{gathered}
\sum_{r=i}^{k-1}(-1)^{1+\overline{\operatorname{deg}}\left(a_{i, r}\right)}\left(\sum_{\sigma_{i} \in S_{a_{i}}} \sum_{\sigma_{i+1} \in \widetilde{S}_{a_{i+1}}} \cdots \sum_{\sigma_{r} \in \widetilde{S}_{a_{r}}} c_{i, r} \chi_{\sigma_{i} \cup \cdots \cup \sigma_{r} \backslash\left(v_{i+1} \cup \cdots \cup v_{r}\right)}\right) . \\
\cdot\left(\sum_{\sigma_{r+1} \in S_{a_{r+1}}} \sum_{\sigma_{r+2} \in \widetilde{S}_{a_{r+2}}} \cdots \sum_{\sigma_{k} \in \widetilde{S}_{a_{k}}} c_{r+1, k} \chi_{\sigma_{r+1} \cup \cdots \cup \sigma_{k} \backslash\left(v_{r+2} \cup \cdots \cup v_{k}\right)}\right)
\end{gathered}
$$

where $c_{i, r}=c_{\sigma_{i}} \ldots c_{\sigma_{r}} \theta_{i, r}$ and $c_{r+1, k}=c_{\sigma_{r+1}} \ldots c_{\sigma_{k}} \theta_{r+1, k}$. For any $\sigma_{r+1} \in$ $S_{a_{r+1}} \backslash \widetilde{S}_{a_{r+1}}$, by definition $\sigma_{r+1} \in P_{a_{r+1}}$ and $\sigma_{i} \cup \sigma_{r+1} \notin \mathcal{K}$. Therefore $\left(\sigma_{i} \cup \cdots \cup \sigma_{r} \backslash\left(v_{i+1} \cup \cdots \cup v_{r}\right)\right) \cup\left(\sigma_{r+1} \cup \cdots \cup \sigma_{k} \backslash\left(v_{r+2} \cup \cdots \cup v_{k}\right)\right) \in \mathcal{K}$ only if $\sigma_{r+1} \in \widetilde{S}_{a_{r+1}}$. Then by expanding the above expression and using the sign from Lemma $2.2, \sum_{r=i}^{k-1} \overline{\overline{a_{i, r}}} \cdot a_{(r+1), k}$ is

$$
\begin{array}{r}
\sum_{r=i}^{k-1} \sum_{\sigma_{i} \in S_{a_{i}}} \sum_{\sigma_{i+1} \in \widetilde{S}_{a_{i+1}}} \cdots \sum_{\begin{array}{c}
\sigma_{k} \in \widetilde{S}_{a_{k}} \\
c_{\sigma_{i}} \ldots c_{\sigma_{k}}
\end{array}}(-1)^{1+\overline{\operatorname{deg}}\left(a_{i, r}\right)+\left|J_{i} \cup \cdots \cup J_{r}\right|\left(p_{r+1}+\cdots+p_{k}+1\right) .} \theta_{r+1, k} \chi_{\sigma_{i} \cup \cdots \cup \sigma_{k} \backslash\left(v_{i+1} \cup \cdots \cup \hat{v}_{r+1} \cup \cdots \cup v_{k}\right)} .
\end{array}
$$

Since $\overline{\operatorname{deg}}\left(a_{i, r}\right)=\left|J_{i} \cup \cdots \cup J_{r}\right|+p_{i}+\cdots+p_{r}+1$,

$$
(-1)^{1+\overline{\operatorname{deg}}\left(a_{i, r}\right)+\left|J_{i} \cup \cdots \cup J_{r}\right|\left(p_{r+1}+\cdots+p_{k}+1\right)}=(-1)^{\left(p_{i}+\cdots+p_{r}\right)+\left|J_{i} \cup \cdots \cup J_{r}\right|\left(p_{r+1}+\cdots+p_{k}\right)} .
$$

We next prove that (3.5) is equal to (3.6) by showing that

$$
\begin{aligned}
\theta_{i, k} \varepsilon\left(v_{r+1}, \sigma_{i} \cup \cdots \cup\right. & \left.\sigma_{k} \backslash\left(v_{i+1} \cup \cdots \cup \hat{v}_{r+1} \cup \cdots \cup v_{k}\right)\right) \\
& =(-1)^{\left(p_{i}+\cdots+p_{r}\right)+\left|J_{i} \cup \cdots \cup J_{r}\right|\left(p_{r+1}+\cdots+p_{k}\right)} \theta_{i, r} \theta_{r+1, k} .
\end{aligned}
$$

Since

$$
\theta_{i, r}=(-1)^{r-i+\left|J_{i}\right|\left(p_{i+1}+\cdots+p_{r}\right)+\cdots+\left|J_{r-1}\right| p_{r}} \varepsilon\left(v_{i+1}, \sigma_{i+1}\right) \cdots \varepsilon\left(v_{r}, \sigma_{r}\right)
$$

and

$$
\theta_{r+1, k}=(-1)^{k-r-1+\left|J_{r+1}\right|\left(p_{r+2}+\cdots+p_{k}\right)+\cdots+\left|J_{k-1}\right| p_{k}} \varepsilon\left(v_{r+2}, \sigma_{r+2}\right) \cdots \varepsilon\left(v_{k}, \sigma_{k}\right)
$$

the right hand side of (3.7) becomes

$$
\begin{aligned}
(-1)^{k-i-1+\left(p_{i}+\cdots+p_{r}\right)+\left|J_{i}\right|\left(p_{i+1}+\cdots+p_{k}\right)+\left|J_{i+1}\right|\left(p_{i+2}+\cdots+p_{k}\right)+\cdots+\left|J_{k-1}\right| p_{k}} \\
\cdot \varepsilon\left(v_{i+1}, \sigma_{i+1}\right) \ldots \varepsilon\left(v_{r}, \sigma_{r}\right) \varepsilon\left(v_{r+2}, \sigma_{r+2}\right) \ldots \varepsilon\left(v_{k}, \sigma_{k}\right) .
\end{aligned}
$$


This is simplified as

$$
(-1)^{p_{i}+\cdots+p_{r}-1} \varepsilon\left(v_{r+1}, \sigma_{r+1}\right) \theta_{i, k} .
$$

Next consider the left hand side of (3.7). For any $r \in\{i, \ldots, k-1\}$, suppose that $v_{r+1} \in \sigma_{r+1}$ is the $l$ th vertex in the vertex set of $\sigma_{i} \cup \cdots \cup \sigma_{k} \backslash\left(v_{i+1} \cup \cdots \cup\right.$ $\left.\hat{v}_{r+1} \cup \cdots \cup v_{k}\right)$. Then by $(2.2)$,

$$
\varepsilon\left(v_{r+1}, \sigma_{i} \cup \cdots \cup \sigma_{k} \backslash\left(v_{i+1} \cup \cdots \cup \hat{v}_{r+1} \cup \cdots \cup v_{k}\right)\right)=(-1)^{l-1} .
$$

Since $v_{r+1} \in \sigma_{r+1}, l$ is given by

$$
l=\left|\sigma_{i}\right|+\left(\left|\sigma_{i+1}\right|-1\right)+\cdots+\left(\left|\sigma_{r}\right|-1\right)+l_{r+1}
$$

where $l_{r+1}$ is the position of $v_{r+1}$ in $\sigma_{r+1}$. Since $\left|\sigma_{i}\right|=p_{i}+1$ for every $i$, $l=\left(p_{i}+1\right)+p_{i+1}+\cdots+p_{r}+l_{r+1}$, and hence

$\varepsilon\left(v_{r+1}, \sigma_{i} \cup \cdots \cup \sigma_{k} \backslash\left(v_{i+1} \cup \cdots \cup \hat{v}_{r+1} \cup \cdots \cup v_{k}\right)\right)=(-1)^{p_{i}+\cdots+p_{r}+1} \varepsilon\left(v_{r+1}, \sigma_{r+1}\right)$.

Thus (3.7) may be rewritten as $(-1)^{p_{i}+\cdots+p_{r}+1} \theta_{i, k} \varepsilon\left(v_{r+1}, \sigma_{r+1}\right)$, which is equal to (3.8). Hence (3.5) is equal to (3.6) so $d\left(a_{i, k}\right)=\sum_{r=i}^{k-1} \overline{\overline{a_{i, r}}} \cdot a_{(r+1), k}$, which proves that $\left(a_{i, k}\right)$ corresponds to a defining system for $\left\langle\alpha_{1}, \ldots, \alpha_{n}\right\rangle$.

We aim to show that the constructed $n$-Massey product $\left\langle\alpha_{1}, \ldots, \alpha_{n}\right\rangle$ is nontrivial. We build a cycle $x \in C_{p_{1}+\ldots+p_{n}+1}\left(\mathcal{K}_{J_{1} \cup \ldots \cup J_{n}}\right)$ and show that for any $[\omega] \in\left\langle\alpha_{1}, \ldots, \alpha_{n}\right\rangle$ there is a cycle $x^{\prime}$ homologous to $x$ such that $\omega\left(x^{\prime}\right) \neq 0$. This will conclude that $[\omega] \neq 0$.

Construction 3.13. Fix $\sigma_{1} \in S_{a_{1}}, \sigma_{i} \in \widetilde{S}_{a_{i}}=S_{a_{i}} \backslash P_{a_{i}}$ for $2 \leqslant i<n$ and $\sigma_{n} \in P_{a_{n}}$. Since $\alpha_{1} \in \widetilde{H}^{p_{1}}\left(\mathcal{K}_{J_{1}}\right)$ is non-zero, there is a cycle $x_{1} \in C_{p_{1}}\left(\mathcal{K}_{J_{1}}\right)$ such that $a_{1}\left(x_{1}\right) \neq 0$. We write the cycle $x_{1}$ as

$$
x_{1}=\sum_{\tilde{\sigma}_{1} \in S_{x_{1}}} c_{\tilde{\sigma}_{1}} \Delta_{\tilde{\sigma}_{1}}
$$

for a collection of $p_{1}$-simplices $S_{x_{1}} \subset \mathcal{K}_{J_{1}}$ and non-zero coefficients $c_{\tilde{\sigma}_{1}}$, where $\Delta_{\tilde{\sigma}_{1}}$ is a basis element of $C_{p_{1}}\left(\mathcal{K}_{J_{1}}\right)$.

After the star deletion of $\sigma_{2} \cup \sigma_{n}$, the boundary complex $\partial\left(\sigma_{2} \cup \sigma_{n}\right)$ is contained in $\mathcal{K}$. Let $x_{2} \in C_{p_{2}+p_{n}}\left(\partial\left(\sigma_{2} \cup \sigma_{n}\right)\right)$ be the cycle

$$
x_{2}=\sum_{w_{2} \in \sigma_{2} \cup \sigma_{n}} c_{w_{2}} \Delta_{\sigma_{2} \cup \sigma_{n} \backslash w_{2}}
$$

for vertices $w_{2} \in \sigma_{2} \cup \sigma_{n}$ and non-zero coefficients $c_{w_{2}}$. Similarly for $3 \leqslant i \leqslant n-1$, let $x_{i} \in C_{p_{i}-1}\left(\partial\left(\sigma_{i}\right)\right)$ be the cycle given by

$$
x_{i}=\sum_{w_{i} \in \sigma_{i}} c_{w_{i}} \Delta_{\sigma_{i} \backslash w_{i}}
$$


for vertices $w_{i} \in \sigma_{i}$ and non-zero coefficients $c_{w_{i}}$.

Let $x \in C_{p_{1}+\ldots+p_{n}+1}\left(\mathcal{K}_{J_{1} \cup \cdots \cup J_{n}}\right)$ be the chain

$$
\begin{aligned}
x=\sum_{\tilde{\sigma}_{1} \in S_{x_{1}}} \sum_{w_{2} \in \sigma_{2} \cup \sigma_{n}} \sum_{w_{3} \in \sigma_{3}} \cdots \sum_{w_{n-1} \in \sigma_{n-1}} c_{\tilde{\sigma}_{1}} c_{w_{2}} \cdots c_{w_{n-1}} \cdot \\
\cdot \Delta_{\tilde{\sigma}_{1} \cup \sigma_{2} \cup \cdots \cup \sigma_{n-1} \cup \sigma_{n} \backslash\left(w_{2} \cup \cdots \cup w_{n-1}\right)} .
\end{aligned}
$$

Let $S_{x}$ be the support of $x$, consisting of simplices

$$
\sigma=\tilde{\sigma}_{1} \cup \sigma_{2} \cup \cdots \cup \sigma_{n-1} \cup \sigma_{n} \backslash\left(w_{2} \cup \cdots \cup w_{n-1}\right)
$$

for a $p_{1}$-simplex $\tilde{\sigma}_{1} \in S_{x_{1}}$, and a choice of vertices $w_{2} \in \sigma_{2} \cup \sigma_{n}, w_{i} \in \sigma_{i}$ for $3 \leqslant i \leqslant n-1$.

Lemma 3.14. The cochain $x \in C_{p_{1}+\ldots+p_{n}+1}\left(\mathcal{K}_{J_{1} \cup \ldots \cup J_{n}}\right)$ is a cycle.

Proof. We show that $x$ is a cycle by explicitly calculating $\partial(x)$. By the definition of the boundary map,

$$
\partial(x)=\sum_{\tilde{\sigma}_{1} \in S_{x_{1}}} \sum_{w_{2} \in \sigma_{2} \cup \sigma_{n}} \sum_{w_{3} \in \sigma_{3}} \cdots \sum_{w_{n-1} \in \sigma_{n-1}} \sum_{v \in \sigma} \varepsilon(v, \sigma) c_{\tilde{\sigma}_{1}} c_{w_{2}} \cdots c_{w_{n-1}} \Delta_{\sigma \backslash v}
$$

where $\sigma \in S_{x}$ as in (3.10). Since $\tilde{\sigma}_{1} \subset J_{1}, \sigma_{i} \subset J_{i}$ for $2 \leqslant i \leqslant n$, and $J_{i} \cap J_{j}=\emptyset$ for $i \neq j$, any choice of vertex $v \in \sigma$ is contained in a simplex $\tilde{\sigma}_{1}$ or $\sigma_{i}$ for $2 \leqslant i \leqslant n$. If $v \in \tilde{\sigma}_{1}$, then $\varepsilon(v, \sigma)=\varepsilon\left(v, \tilde{\sigma}_{1}\right)$. Also if $v \in \sigma_{i}$ for $i>1$, then

$$
\varepsilon(v, \sigma)= \begin{cases}(-1)^{p_{1}+1} \varepsilon\left(v, \sigma_{2}\right) & \text { if } w_{2} \in \sigma_{n} \text { and } i=2, \\ (-1)^{p_{1}+\cdots+p_{i-1}+2} \varepsilon\left(v, \sigma_{i} \backslash \tilde{w}_{i}\right) & \text { if } w_{2} \in \sigma_{n} \text { and } i>2, \\ (-1)^{p_{1}+\cdots+p_{n-1}+1} \varepsilon\left(v, \sigma_{n}\right) & \text { if } w_{2} \in \sigma_{2} \text { and } i=n, \\ (-1)^{p_{1}+\cdots+p_{i-1}+1} \varepsilon\left(v, \sigma_{i} \backslash w_{i}\right) & \text { if } w_{2} \in \sigma_{2} \text { and } i<n\end{cases}
$$

where $\tilde{w}_{i}=w_{i}$ for $1<i<n$, and $\tilde{w}_{n}=w_{2}$. We rewrite $\partial(x)$ as

$$
\partial(x)=\sum_{\tilde{\sigma}_{1} \in S_{x_{1}}} \sum_{w_{2} \in \sigma_{2} \cup \sigma_{n}} \sum_{w_{3} \in \sigma_{3}} \ldots \sum_{w_{n-1} \in \sigma_{n-1}} \sum_{i=1}^{n} \sum_{v \in \tilde{\sigma}_{i} \backslash \tilde{w}_{i}} \varepsilon(v, \sigma) c_{\tilde{\sigma}_{1}} c_{w_{2}} \cdots c_{w_{n-1}} \Delta_{\sigma \backslash v}
$$

where $\tilde{\sigma}_{1} \backslash \tilde{w}_{1}=\tilde{\sigma}_{1}$ and $\tilde{\sigma}_{i}=\sigma_{i}$ for $i>1$. Let $\Delta_{\sigma \backslash v \mid J}$ denote the restriction of $\Delta_{\sigma \backslash v}$ to its vertices in $J \subset V(\mathcal{K})$, where $V(\mathcal{K})$ is the vertex set of $\mathcal{K}$. Then

$$
\begin{aligned}
\partial(x)=\sum_{\tilde{\sigma}_{1} \in S_{x_{1}}} \sum_{w_{2} \in \sigma_{2} \cup \sigma_{n}} \sum_{w_{3} \in \sigma_{3}} \ldots \sum_{w_{n-1} \in \sigma_{n-1}} \\
\left(\sum_{i=1}^{n} \sum_{v \in \tilde{\sigma}_{i} \backslash \tilde{w}_{i}} \varepsilon(v, \sigma) c_{\tilde{\sigma}_{1}} c_{w_{2}} \cdots c_{w_{n-1}}\left(\Delta_{\sigma \backslash v \mid J_{i}}\right)\left(\Delta_{\sigma \backslash v \mid V(\mathcal{K}) \backslash J_{i}}\right)\right) .
\end{aligned}
$$

We rearrange $\partial(x)$ into four sums, one in which $v \in \tilde{\sigma}_{1}$, another for $v \in \sigma_{2} \cup \sigma_{n} \backslash w_{2}$, and two more when $v \in \sigma_{i} \backslash w_{i}$ for $3 \leqslant i \leqslant n-1$ where either $w_{2} \in \sigma_{2}$ or $w_{2} \in \sigma_{2}$. 
Then expanding $\varepsilon(v, \sigma), \partial(x)$ is

$$
\begin{aligned}
& \sum_{w_{2} \in \sigma_{2} \cup \sigma_{n}} \sum_{w_{3} \in \sigma_{3}} \cdots \sum_{w_{n-1} \in \sigma_{n-1}} c_{w_{2}} \cdots c_{w_{n-1}} . \\
& \cdot\left(\Delta_{\sigma \backslash v \mid V(\mathcal{K}) \backslash J_{1}}\right)\left(\sum_{\tilde{\sigma}_{1} \in S_{x_{1}}} \sum_{v \in \tilde{\sigma}_{1}} \varepsilon\left(v, \tilde{\sigma}_{1}\right) c_{\tilde{\sigma}_{1}}\left(\Delta_{\sigma \backslash v \mid J_{1}}\right)\right)+ \\
& +\sum_{\tilde{\sigma}_{1} \in S_{x_{1}}} \sum_{w_{3} \in \sigma_{3}} \cdots \sum_{w_{n-1} \in \sigma_{n-1}} c_{\tilde{\sigma}_{1}} c_{w_{3}} \cdots c_{w_{n-1}}(-1)^{p_{1}+p_{3}+\cdots+p_{n-1}+1}\left(\Delta_{\sigma \backslash v \mid V(\mathcal{K}) \backslash J_{2} \cup J_{n}}\right) . \\
& \cdot\left(\sum_{w_{2} \in \sigma_{2} \cup \sigma_{n}} \sum_{v \in \sigma_{2} \cup \sigma_{n} \backslash w_{2}} \varepsilon\left(v, \sigma_{2} \cup \sigma_{n} \backslash w_{2}\right) c_{w_{2}}\left(\Delta_{\sigma \backslash v \mid J_{2} \cup J_{n}}\right)\right)+ \\
& +\sum_{\tilde{\sigma}_{1} \in S_{x_{1}}} \sum_{w_{2} \in \sigma_{2}} \sum_{w_{3} \in \sigma_{3}} \cdots \sum_{w_{n-1} \in \sigma_{n-1}} c_{\tilde{\sigma}_{1}} c_{w_{2}} \cdots c_{w_{n-1}} . \\
& \cdot\left(\sum_{i=3}^{n-1}(-1)^{p_{1}+\cdots+p_{i-1}+1}\left(\Delta_{\sigma \backslash v \mid V(\mathcal{K}) \backslash J_{i}}\right)\left(\sum_{v \in \sigma_{i} \backslash w_{i}} \varepsilon\left(v, \sigma_{i} \backslash w_{i}\right)\left(\Delta_{\sigma \backslash v \mid J_{i}}\right)\right)\right)+ \\
& +\sum_{\tilde{\sigma}_{1} \in S_{x_{1}}} \sum_{w_{2} \in \sigma_{n}} \sum_{w_{3} \in \sigma_{3}} \cdots \sum_{w_{n-1} \in \sigma_{n-1}} c_{\tilde{\sigma}_{1}} c_{w_{2}} \cdots c_{w_{n-1}} . \\
& \cdot\left(\sum_{i=3}^{n-1}(-1)^{p_{1}+\cdots+p_{i-1}+2}\left(\Delta_{\sigma \backslash v \mid V(\mathcal{K}) \backslash J_{i}}\right)\left(\sum_{v \in \sigma_{i} \backslash w_{i}} \varepsilon\left(v, \sigma_{i} \backslash w_{i}\right)\left(\Delta_{\sigma \backslash v \mid J_{i}}\right)\right)\right) .
\end{aligned}
$$

Each sum can be written in terms of $\partial\left(x_{i}\right)$, that is,

$$
\begin{aligned}
\partial(x)= & \sum_{w_{2} \in \sigma_{2} \cup \sigma_{n}} \sum_{w_{3} \in \sigma_{3}} \ldots \sum_{w_{n-1} \in \sigma_{n-1}} c_{w_{2}} \cdots c_{w_{n-1}}\left(\Delta_{\sigma \backslash v \mid V(\mathcal{K}) \backslash J_{1}}\right) \partial\left(x_{1}\right)+ \\
& +\sum_{\tilde{\sigma}_{1} \in S_{x_{1}}} \sum_{w_{3} \in \sigma_{3}} \cdots \sum_{w_{n-1} \in \sigma_{n-1}} c_{\tilde{\sigma}_{1}} c_{w_{3}} \cdots c_{w_{n-1}} \cdot \\
& +\sum_{\tilde{\sigma}_{1} \in S_{x_{1}}} \sum_{w_{2} \in \sigma_{2}} \sum_{i=3}^{n-1} \sum_{w_{3} \in \sigma_{3}} \cdots \widehat{\sum_{w_{i} \in \sigma_{i}}} \cdots \sum_{w_{n-1} \in \sigma_{n-1}} c_{\tilde{\sigma}_{1}} c_{w_{2}} \cdots \widehat{c_{w_{i}}} \cdots c_{w_{n-1}} \cdot \\
& +\sum_{\tilde{\sigma}_{1} \in S_{x_{1}}} \sum_{w_{2} \in \sigma_{n}} \sum_{i=3}^{n-1} \sum_{w_{3} \in \sigma_{3}} \cdots \widehat{\sum_{w_{i} \in \sigma_{i}}} \cdots \sum_{w_{n-1} \in \sigma_{n-1}} c_{\tilde{\sigma}_{1} c_{w_{2}}} \cdots \widehat{c_{w_{i}}} \cdots c_{w_{n-1}} \cdot \\
&
\end{aligned}
$$

where ${ }^{-}$denotes omission. Since $\partial\left(x_{i}\right)=0$ for every $i, x$ is a cycle as well.

Example 3.15. Let $\mathcal{K}$ be the simplicial complex in Figure 6a, where the simplices $\sigma_{1} \cup \sigma_{2}^{\prime}, \sigma_{2} \cup \sigma_{3}^{\prime}$ were star deleted and $S_{a_{1}}=\left\{\sigma_{1}\right\}, S_{a_{2}}=\left\{\sigma_{2}\right\}, S_{a_{3}}=\left\{\sigma_{3}\right\}$, $P_{a_{2}}=\left\{\sigma_{2}^{\prime}\right\}, P_{a_{3}}=\left\{\sigma_{3}^{\prime}\right\}$. The cycle $x$ is supported on simplices of the form

$$
\sigma=\tilde{\sigma}_{1} \cup \sigma_{2} \cup \sigma_{3}^{\prime} \backslash\left(w_{2}\right)
$$


where $\tilde{\sigma}_{1}$ is either $\sigma_{1}$ or $\sigma_{1}^{\prime}$ and $w_{2} \in \sigma_{2} \cup \sigma_{3}^{\prime}$. Therefore $S_{x}$ contains $\sigma_{1} \cup \sigma_{2}$, $\sigma_{1}^{\prime} \cup \sigma_{2}, \sigma_{1}^{\prime} \cup \sigma_{3}^{\prime}$ and $\sigma_{1} \cup \sigma_{3}^{\prime}$, as shown in Figure 6b.

If $a_{1}=\chi_{\sigma_{1}} \in C^{0}\left(\mathcal{K}_{\sigma_{1}, \sigma_{1}^{\prime}}\right), a_{2}=\chi_{\sigma_{2}} \in C^{0}\left(\mathcal{K}_{\sigma_{2}, \sigma_{2}^{\prime}}\right)$ and $a_{3}=\chi_{\sigma_{3}} \in$ $C^{0}\left(\mathcal{K}_{\sigma_{3}, \sigma_{3}^{\prime}}\right)$, then the rest of the defining system constructed in Proposition 3.12 is $a_{12}=-\chi_{\sigma_{1}}$ and $a_{23}=-\chi_{\sigma_{2}}$. The associated cocycle to this defining system is

$$
\omega=-\chi_{\sigma_{1} \cup \sigma_{3}}-\chi_{\sigma_{1} \cup \sigma_{2}} .
$$

There is exactly one simplex $\sigma_{1} \cup \sigma_{2}=S_{x} \cap S_{\omega}$. So by evaluating $\omega$ on $x$, $\omega(x) \neq 0$.

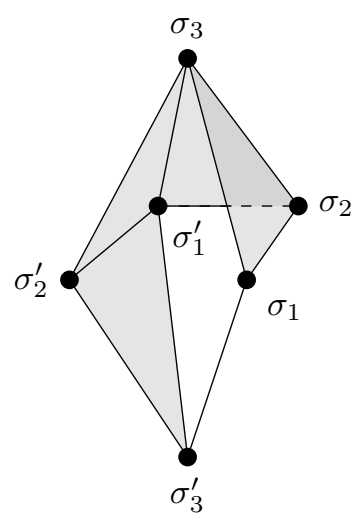

(a) A simplicial complex $\mathcal{K}$ constructed by star deletions at $\sigma_{1} \cup \sigma_{2}^{\prime}$ and $\sigma_{2} \cup \sigma_{3}^{\prime}$.

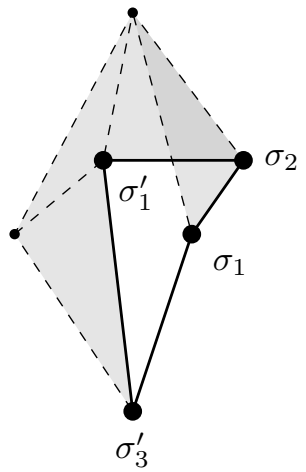

(b) The cycle $x$.

Figure 6

Proposition 3.16. The $n$-Massey product $\left\langle\alpha_{1}, \ldots, \alpha_{n}\right\rangle \subset H^{*}\left(\mathcal{Z}_{\mathcal{K}}\right)$ is nontrivial.

Proof. For any $[\omega] \in\left\langle\alpha_{1}, \ldots, \alpha_{n}\right\rangle$, we consider a corresponding cocycle $\omega \in$ $C^{p_{1}+\cdots+p_{n}+1}\left(\mathcal{K}_{J_{1} \cup \cdots \cup J_{n}}\right)$ with the cycle $x$ from Construction 3.13 and aim to show that $S_{\omega} \cap S_{x}$ contains only one simplex. This implies that $\omega(x)$ is nonzero, and therefore $[\omega] \neq 0$.

First we define a subcollection of simplices in $S_{\omega}$. Let $\left(a_{i, k}\right)$ be any defining system of $\left\langle\alpha_{1}, \ldots, \alpha_{n}\right\rangle$. Let $S_{a_{i, k}}$ be the support of $a_{i, k}$ so that

$$
a_{i, k}=\sum_{\sigma \in S_{a_{i, k}}} c_{\sigma} \chi_{\sigma}
$$

for non-zero coefficients $c_{\sigma} \in \mathbf{k}$. The image of the coboundary map is a cochain obtained by adding a vertex to the simplices in $S_{a_{i, k}}$. Since $d\left(a_{i, k}\right)=$ $\sum_{r=i}^{k-1} \overline{\overline{a_{i, r}}} a_{r+1, k}$, for any $\sigma_{i, r} \in S_{a_{i, r}}$ and $\sigma_{r+1, k} \in S_{a_{r+1, k}}$ there is a simplex $\sigma \in S_{a_{i, k}}$ and vertex $u_{i} \in \sigma_{i, r} \cup \sigma_{r+1, k}$ such that $\sigma=\sigma_{i, r} \cup \sigma_{r+1, k} \backslash u_{i}$. We extend this principle to say that there is a simplex $\sigma \in S_{a_{2, n}}$ such that 
$\sigma=\sigma_{2} \cup \cdots \cup \sigma_{n} \backslash\left(u_{2} \cup \cdots \cup u_{n-1}\right)$ for $\sigma_{i} \in \widetilde{S}_{a_{i}}=S_{a_{i}} \backslash P_{a_{i}}$ and vertices $u_{i} \in \sigma_{2} \cup \cdots \cup \sigma_{n}$ for $2 \leqslant i \leqslant n, u_{i} \neq u_{j}$. Let $\omega$ be the associated cocycle for this defining system,

$$
\omega=\sum_{\tau \in S_{\omega}} c_{\tau} \chi_{\tau}
$$

for non-zero coefficients $c_{\tau} \in \mathbf{k}$. The support of the first summand $\overline{\bar{a}}_{1} a_{2, n}$ of $\omega$ contains a simplex of the form

$$
\tau=\sigma_{1} \cup \sigma_{2} \cup \cdots \cup \sigma_{n-1} \cup \sigma_{n} \backslash\left(u_{2} \cup \cdots \cup u_{n-1}\right)
$$

for $\sigma_{1} \in S_{a_{1}}, \sigma_{i} \in \widetilde{S}_{a_{i}}$ and vertices $u_{i} \in \sigma_{2} \cup \cdots \cup \sigma_{n}$ for $2 \leqslant i \leqslant n, u_{i} \neq u_{j}$. Hence $\tau \in S_{\omega}$.

We compare the simplices $\tau \in S_{\omega}$ in (3.11) and $\sigma \in S_{x}$ in (3.10) where

$$
\sigma=\tilde{\sigma}_{1} \cup \sigma_{2} \cup \cdots \cup \sigma_{n-1} \cup \sigma_{n}^{\prime} \backslash\left(w_{2} \cup \cdots \cup w_{n-1}\right)
$$

for $\tilde{\sigma}_{1} \in S_{x_{1}}, \sigma_{i} \in \widetilde{S}_{a_{i}}$ for $i \in\{2, \ldots, n-1\}, \sigma_{n}^{\prime} \in P_{a_{n}}$ and a choice of vertices $w_{2} \in \sigma_{2} \cup \sigma_{n}, w_{i} \in \sigma_{i}$ for $3 \leqslant i \leqslant n-1$. For $\sigma_{1} \in S_{a_{1}}$ and $\sigma_{i} \in \widetilde{S}_{a_{i}}$ for $2 \leqslant i \leqslant n$, the simplex $\sigma_{1} \cup \cdots \cup \sigma_{n} \in \mathcal{K}$ was not removed by star deletion in Construction 3.5. Both $\tau$ and $\sigma$ are $\left(p_{1}+\cdots+p_{n}+1\right)$-dimensional faces of $\sigma_{1} \cup \cdots \cup \sigma_{n}$. If there is no $\tau \in S_{\omega}$ and $\sigma \in S_{x}$ such that $\tau=\sigma$, then there is a cochain $b \in C^{p_{1}+\cdots+p_{n}}(\mathcal{K})$ whose support consists of $\left(p_{1}+\cdots+p_{n}\right)$-simplices contained in $\sigma_{1} \cup \cdots \cup \sigma_{n}$ and the support of $d(b)$ contains both $\tau$ and $\sigma$. Let $\omega^{\prime}=\omega+c_{\tau} c_{d(b), \sigma} d(b)$ where $c_{\tau}$ is the coefficient of $\tau \in S_{\omega}$ and $c_{d(b), \sigma}$ is the coefficient of $\sigma \in S_{d(b)}$. Then $S_{\omega^{\prime}}$ contains $\sigma$ and does not contain $\tau$. Therefore $\sigma \in S_{\omega^{\prime}} \cap S_{x}$. However there could be other simplices in $S_{\omega^{\prime}} \cap S_{x}$ that cancel, so we cannot conclude that $\omega^{\prime}(x)$ is non-zero. To resolve this, we change the representatives of $[\omega]$ and $[x]$ so that there is only one term in their evaluation.

Suppose that there is $\tau^{\prime} \in S_{\omega^{\prime}} \cap S_{x}, \tau \neq \tau^{\prime}$. If $\operatorname{lk}_{\mathcal{K}}\left(\tau^{\prime}\right) \neq \emptyset$, then there is a $\left(p_{1}+\ldots+p_{k}+2\right)$-dimensional simplex $A \in \mathcal{K}_{J_{1} \cup \ldots \cup J_{n}}$ containing $\tau^{\prime}$ in its boundary. Suppose that $S_{\omega^{\prime}}$ does not contain an additional face of $A$. Then replace $x$ by $x^{\prime}$, where the simplex $\tau^{\prime} \in S_{x}$ is replaced by the $\left(p_{1}+\ldots+p_{k}+1\right)$ simplices in $\partial(A) \backslash \tau^{\prime}$ to form $S_{x^{\prime}}$ as illustrated in Figure 7. Therefore $x^{\prime}$ is the cycle $x-c_{\tau^{\prime}} \epsilon(v, A) \partial\left(\Delta_{A}\right)$, where $c_{\tau^{\prime}}$ is the coefficient of the summand $\Delta_{\tau^{\prime}}$ in $x, v$ is the vertex such that $v \cup \tau^{\prime}=A$, and $\epsilon(v, A)$ is the coefficient of $\Delta_{\tau}$ in $\partial\left(\Delta_{A}\right)$. Thus $[x]=\left[x^{\prime}\right]$ and $\tau^{\prime} \notin S_{\omega^{\prime}} \cap S_{x^{\prime}}$.

Alternatively, suppose that $\operatorname{lk}_{\mathcal{K}}\left(\tau^{\prime}\right)=\emptyset$, or $\operatorname{lk}_{\mathcal{K}}\left(\tau^{\prime}\right) \neq \emptyset$ and $S_{\omega^{\prime}}$ contains an additional face $\tau^{\prime \prime}$ of $A$. Since $x$ is a cycle, there is another simplex $t \neq$ $\tau^{\prime} \in S_{x}$ such that $\tau^{\prime} \cap t \neq \emptyset$ (as shown in Figure 7c). Let $\omega^{\prime \prime}=\omega^{\prime}-c_{\tau^{\prime}} \varepsilon\left(\tau^{\prime} \backslash\right.$ $\left.\tau^{\prime} \cap t, \tau^{\prime}\right) d\left(\chi_{\tau^{\prime} \cap t}\right)$ where $c_{\tau^{\prime}}$ is the coefficient of the summand $\chi_{\tau^{\prime}}$ in $\omega^{\prime}$ and $\varepsilon\left(\tau^{\prime} \backslash \tau^{\prime} \cap t, \tau^{\prime}\right)$ is its coefficient in $d\left(\chi_{\tau^{\prime} \cap t}\right)$. So $\left[\omega^{\prime \prime}\right]=\left[\omega^{\prime}\right]$ and $S_{\omega^{\prime \prime}}$ contains $t$ but $S_{\omega^{\prime \prime}} \cap S_{x}$ does not contain $\tau^{\prime}$.

By this process of replacing simplices in the intersection of the supports one-by-one, we obtain a cocycle $\omega^{\prime} \in C^{p_{1}+\ldots+p_{n}+1}\left(\mathcal{K}_{J_{1} \cup \ldots \cup J_{n}}\right)$ and a cycle $x^{\prime} \in$ $C_{p_{1}+\ldots+p_{n}+1}\left(\mathcal{K}_{J_{1} \cup \ldots \cup J_{n}}\right)$ such that $\left[\omega^{\prime}\right]=[\omega],\left[x^{\prime}\right]=[x]$ and $S_{\omega^{\prime}} \cap S_{x^{\prime}}$ contains only one simplex. Thus $\omega^{\prime}\left(x^{\prime}\right) \neq 0$, and so $\left[\omega^{\prime}\right]=[\omega]$ is non-zero. 


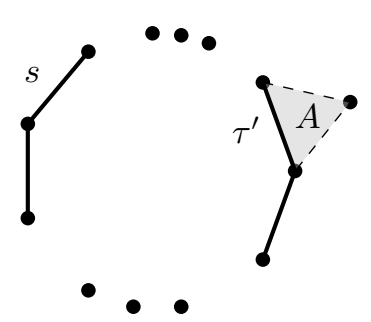

(a) The cycle $x$

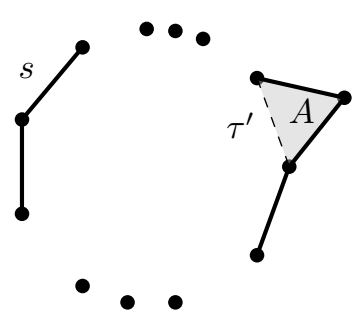

(b) The cycle $x^{\prime}$

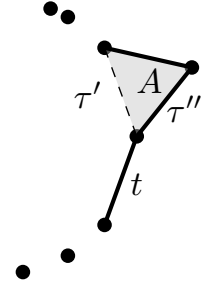

(c) There is a simplex $t \in S_{x}$ that shares a boundary with

Figure 7: If the link of $\tau^{\prime}$ is non-empty, then the cycle $x$ can be changed to $x^{\prime}$

By combining Propositions 3.12 and 3.16, we have proved the main theorem.

Theorem 3.17. For $i \in\{1, \ldots, n\}$, let $\mathcal{K}^{i}$ be a simplicial complex on $\left[m_{i}\right]$ that is not an $\left(m_{i}-1\right)$-simplex. Then there exists a simplicial complex $\mathcal{K}$, obtained by performing star deletions on $\mathcal{K}^{1} * \cdots * \mathcal{K}^{n}$, with a non-trivial n-Massey product in $H^{*}\left(\mathcal{Z}_{\mathcal{K}}\right)$.

Example 3.18. For $i=1,2,3$, let $\mathcal{K}^{i}$ be the simplicial complexes as in Example 3.7 and let

$$
\mathcal{K}=\operatorname{sd}_{\{5,8\}} \operatorname{sd}_{\{4,8\}} \operatorname{sd}_{\{3,8\}} \operatorname{sd}_{\{1,6\}} \operatorname{sd}_{\{1,5\}} \operatorname{sd}_{\{1,4\}} \mathcal{K}^{1} * \mathcal{K}^{2} * \mathcal{K}^{3} .
$$

Suppose that $a_{1}=\chi_{1} \in C^{0}\left(\mathcal{K}^{1}\right), a_{2}=\chi_{3}+\chi_{4}+\chi_{5} \in C^{0}\left(\mathcal{K}^{2}\right), a_{3}=\chi_{7} \in C^{0}\left(\mathcal{K}^{3}\right)$. Then $S_{a_{1}}=\{1\}, S_{a_{2}}=\{\{3\},\{4\},\{5\}\}, S_{a_{3}}=\{\{7\}\}$ and $P_{a_{2}}=\{4,5,6\}$, $P_{a_{3}}=\{8\}$. The rest of the defining system constructed in (3.2) is

$$
\begin{aligned}
& a_{1,2}=\theta_{1,2} \chi_{1}=-\chi_{1} \\
& a_{2,3}=\theta_{2,3}\left(\chi_{3}+\chi_{4}+\chi_{5}\right)=-\left(\chi_{3}+\chi_{4}+\chi_{5}\right) .
\end{aligned}
$$

The associated cocycle $\omega$ for this defining system is

$$
\omega=-\chi_{1}\left(\chi_{3}+\chi_{4}+\chi_{5}\right)-\chi_{1} \chi_{7}
$$

Therefore $\omega \in C^{1}(\mathcal{K})$ evaluates non-trivially on the 1-cycle $x=\Delta_{\{1,3\}}-\Delta_{\{2,3\}}+$ $\Delta_{\{2,8\}}-\Delta_{\{1,8\}}$. Another defining system could have $a_{2,3}^{\prime}=\chi_{8}+\chi_{6}+\chi_{7}$. Then the associated cocycle $\omega^{\prime}$ for this defining system is given by

$$
\omega^{\prime}=\chi_{1}\left(\chi_{6}+\chi_{7}+\chi_{8}\right)+-\chi_{1} \chi_{7}=\chi_{17}+\chi_{18}-\chi_{17}=\chi_{18} .
$$

Thus $\omega^{\prime}$ also evaluates non-trivially on $x$. By Proposition 3.16, the associated cocycle of any defining system evaluates non-trivially on some cycle. Hence $\left\langle\left[a_{1}\right],\left[a_{2}\right],\left[a_{3}\right]\right\rangle \subset H^{10}\left(\mathcal{Z}_{\mathcal{K}}\right)$ is a non-trivial Massey product.

Two particular examples of Theorem 3.17 are the families of Baskakov and Limonchenko. 
Example 3.19 (Baskakov's family [5]). For $i=1,2,3$, let $\mathcal{K}^{i}$ be a triangulation of a $\left(n_{i}-1\right)$-sphere on $\left[m_{i}\right]$. Let $\sigma_{1} \in \mathcal{K}^{1}, \sigma_{2}, \sigma_{2}^{\prime} \in \mathcal{K}^{2}, \sigma_{3} \in \mathcal{K}^{3}$ be maximal simplices such that $\sigma_{2}$ and $\sigma_{2}^{\prime}$ are adjacent, that is, there is a vertex $v_{2^{\prime}} \in \mathcal{K}^{2}$ such that $\left(\sigma_{2} \cap \sigma_{2}^{\prime}\right) \cup v_{2^{\prime}}=\sigma_{2}^{\prime}$. Similarly, let $\sigma_{3}^{\prime} \in \mathcal{K}^{3}$ be a maximal simplex adjacent to $\sigma_{3}$ so that there exists a vertex $v_{3^{\prime}} \in \mathcal{K}^{3}$ such that $\left(\sigma_{3} \cap \sigma_{3}^{\prime}\right) \cup v_{3^{\prime}}=\sigma_{3}^{\prime}$. Let $a_{1}=\chi_{\sigma_{1}}, a_{2}=\chi_{\sigma_{2^{\prime}}}$, and $a_{3}=\chi_{\sigma_{3^{\prime}}}$ be cocycle representatives of $\alpha_{i} \in$ $\widetilde{H}^{n_{i}-1}\left(\mathcal{K}^{i}\right)$ for $i=1,2,3$. Baskakov [5] constructed $\mathcal{K}^{\prime}=\operatorname{ss}_{\left\{\sigma_{1}, \sigma_{2^{\prime}}\right\}} \operatorname{ss}_{\left\{\sigma_{2}, \sigma_{3}\right\}} \mathcal{K}^{1} *$ $\mathcal{K}^{2} * \mathcal{K}^{3}$ and showed that $\left\langle\alpha_{1}, \alpha_{2}, \alpha_{3}\right\rangle$ is a non-trivial Massey product in $H^{*}\left(\mathcal{Z}_{\mathcal{K}}\right)$ where $\mathcal{K}$ is the restriction of $\mathcal{K}^{\prime}$ to the vertex set $\left[m_{1}\right] \cup\left[m_{2}\right] \cup\left[m_{3}\right]$. Since $\mathcal{K}=\operatorname{sd}_{\left\{\sigma_{1}, \sigma_{2^{\prime}}\right\}} \operatorname{sd}_{\left\{\sigma_{2}, \sigma_{3}\right\}} \mathcal{K}^{1} * \mathcal{K}^{2} * \mathcal{K}^{3}$, Theorem 3.17 recovers Baskakov's family of examples of non-trivial triple Massey products in $H^{*}\left(\mathcal{Z}_{\mathcal{K}}\right)$. The simplest example when $\mathcal{K}^{1}, \mathcal{K}^{2}, \mathcal{K}^{3}$ are $S^{0}$ is shown in Figure 8 and its restriction to the original 6 vertices is in Figure 6 a after swapping the labels $\sigma_{3}, \sigma_{3^{\prime}}$

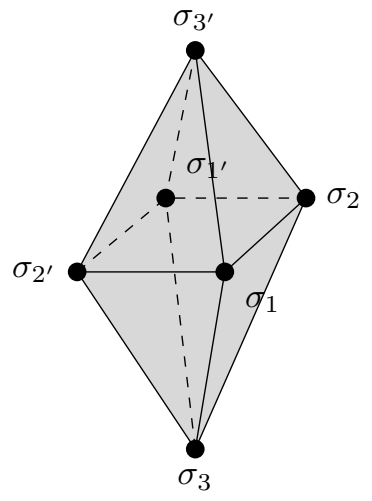

(a) $\mathcal{K}^{1} * \mathcal{K}^{2} * \mathcal{K}^{3}$

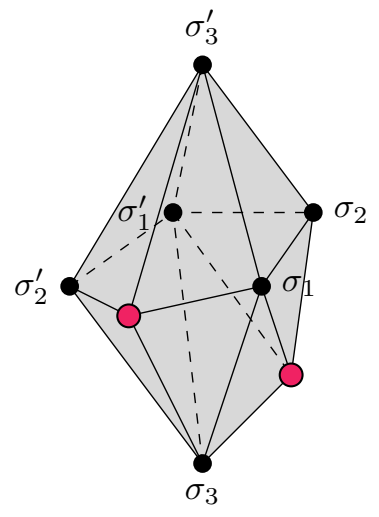

(b) $\operatorname{ss}_{\left\{\sigma_{1}, \sigma_{2}\right\}} \operatorname{ss}_{\left\{\sigma_{2}, \sigma_{3}\right\}} \mathcal{K}^{1} * \mathcal{K}^{2} * \mathcal{K}^{3}$ with cone vertices coloured

Figure 8: The simplest example of both Baskakov and Limonchenko's families of non-trivial Massey products in moment-angle complexes

Example 3.20 (Limonchenko's family [18]). Let $F$ be a face of a polytope $P$ and suppose that there is a hyperplane $H$ that does not include any vertices of $P$ but separates the vertices of $F$ from the other vertices in $P$. If $H_{1}, H_{2}$ are the half spaces defined by $H$ and $F \subset H_{2}$, then the polytope $P \cap H_{1}$ is called a truncation of $P$ at $F$. A family of non-trivial $n$-Massey products is constructed by truncating the unit $n$-cube $I^{n}=I \times \cdots \times I$ as follows. Suppose that opposite facets of $I^{n}$ are labelled $F_{l}, F_{l^{\prime}}$ for $l=1, \ldots, n$. The boundary of the dual $\mathcal{K}=\mathcal{K}_{I^{n}}=\partial\left(I^{n}\right)^{*}$ is the join of $n$ copies of $S^{0}$, for example $\mathcal{K}_{I^{3}}$ is shown in Figure 8a. To create a non-trivial $n$-Massey product, Limonchenko [18, Construction 1] truncated $I^{n}$ at the intersection of facets $F_{i}$ and $F_{k^{\prime}}$ for $1 \leqslant i<$ $k \leqslant n,(i, k) \neq(1, n)$. For example see Figure 9. These truncations correspond to stellar subdividing $\mathcal{K}_{I^{n}}$ at the edges $\sigma_{i} \cup \sigma_{k^{\prime}}$, where $\sigma_{l}, \sigma_{l^{\prime}} \in \mathcal{K}_{I^{n}}$ are the 


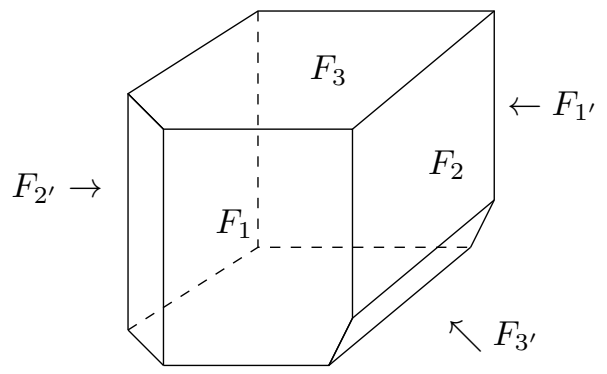

Figure 9: A 3-cube truncated at the faces $F_{1} \cap F_{2^{\prime}}$ and $F_{2} \cap F_{3^{\prime}}$, which is dual to Figure 8b with the labels $\sigma_{3}, \sigma_{3^{\prime}}$ swapped.

vertices that are dual to the facets $F_{l}, F_{l^{\prime}}$ in $I^{n}$. Let $\mathcal{K}$ be the restriction of the stellar subdivided complex to the $2 n$ vertices $\sigma_{l}, \sigma_{l^{\prime}}$ for $l=1, \ldots, n$, and let $\alpha_{l}$ be the generator of $\widetilde{H}^{0}\left(\mathcal{K}_{\sigma_{l}, \sigma_{l^{\prime}}}\right)$. Limonchenko showed that the $n$ Massey product $\left\langle\alpha_{1}, \ldots, \alpha_{n}\right\rangle \subset H^{*}\left(\mathcal{Z}_{\mathcal{K}}\right)$ is non-trivial. Since this construction is recovered by star deleting $K_{I^{n}}$ as described in Construction 3.5, Theorem 3.17 gives an alternative proof that $\left\langle\alpha_{1}, \ldots, \alpha_{n}\right\rangle$ is non-trivial.

Theorem 3.17 does not just give alternative proofs of existing results about non-trivial Massey products in the cohomology of moment-angle complexes, it creates non-trivial $n$-Massey products from any non-zero cohomology classes supported on a full subcomplex of any simplicial complex $\mathcal{K}^{i}$. Therefore there is no limit on $n$ or the dimension of the classes $\alpha_{i}$. Using this construction it is also possible to construct Massey products on torsion elements.

Example 3.21. Let $\mathcal{K}^{1}$ be a triangulation of $\mathbb{R} P^{2}$ on 6 vertices as in Figure 10. Let $\mathcal{K}^{2}, \mathcal{K}^{3}$ be copies of two disjoint vertices labelled 6,7 and 8,9, respectively. Let $\alpha_{1} \in \widetilde{H}^{2}\left(\mathcal{K}^{1}\right)$ be represented by $\chi_{012}$. For $i=2,3$, let $\alpha_{i} \in \widetilde{H}^{0}\left(\mathcal{K}^{i}\right)$ be represented by $a_{2}=\chi_{6}$ and $a_{3}=\chi_{8}$, respectively. By Construction 3.5, $P_{a_{2}}=$ $\{\{7\}\}$ and $P_{a_{3}}=\{\{9\}\}$. Then let

$$
\mathcal{K}=\operatorname{sd}_{\{0127\}} \operatorname{sd}_{\{69\}} \mathcal{K}^{1} * \mathcal{K}^{2} * \mathcal{K}^{3} .
$$

By Theorem 3.17, there is a non-trivial triple Massey product $\left\langle\alpha_{1}, \alpha_{2}, \alpha_{3}\right\rangle \subset$ $H^{14}\left(\mathcal{Z}_{\mathcal{K}}\right)$. This is the smallest example of a non-trivial triple Massey product on a torsion class since $\mathcal{K}^{1}$ is the triangulation of $\mathbb{R} P^{2}$ on the least number of vertices.

Since $\alpha_{1}$ is the generator of $\widetilde{H}^{2}\left(\mathcal{K}^{1}\right) \cong \widetilde{H}^{2}\left(\mathbb{R} P^{2}\right), \alpha_{1}$ is a torsion element. The associated cocycle for the defining system constructed in (3.2) is $\omega=-\chi_{0126}-$ $\chi_{0128} \in C^{3}(\mathcal{K})$. The corresponding class $[\omega] \in\left\langle\alpha_{1}, \alpha_{2}, \alpha_{3}\right\rangle$ is not a torsion element in $H^{14}\left(\mathcal{Z}_{\mathcal{K}}\right)$.

Also, there is a cochain $a_{1,2}^{\prime}=\chi_{126}+\chi_{124}-\chi_{147}-\chi_{347}+\chi_{037}+\chi_{027}$ such that $d\left(a_{1,2}^{\prime}\right)=\chi_{0126} \in C^{3}\left(\mathcal{K}_{01234567}\right)$, which is different to $a_{1,2}$ constructed in (3.2). The associated cocycle to this defining system is $\omega^{\prime}=-\chi_{0126}+\chi_{1268}+\chi_{1248}-$ $\chi_{1478}-\chi_{3478}+\chi_{0378}+\chi_{0278}$ with $\left[\omega^{\prime}\right] \neq 0$ and $[\omega] \neq\left[\omega^{\prime}\right]$. Therefore $\left\langle\alpha_{1}, \alpha_{2}, \alpha_{3}\right\rangle$ 
has non-trivial indeterminacy. In particular, the indeterminacy is given by $\alpha_{1}$. $\widetilde{H}^{0}\left(\mathcal{K}_{6789}\right)+\alpha_{3} \cdot \widetilde{H}^{2}\left(\mathcal{K}_{01234567}\right)=\alpha_{3} \cdot \widetilde{H}^{2}\left(\mathcal{K}_{01234567}\right)$, where $\widetilde{H}^{2}\left(\mathcal{K}_{01234567}\right) \cong \mathbb{Z}$.

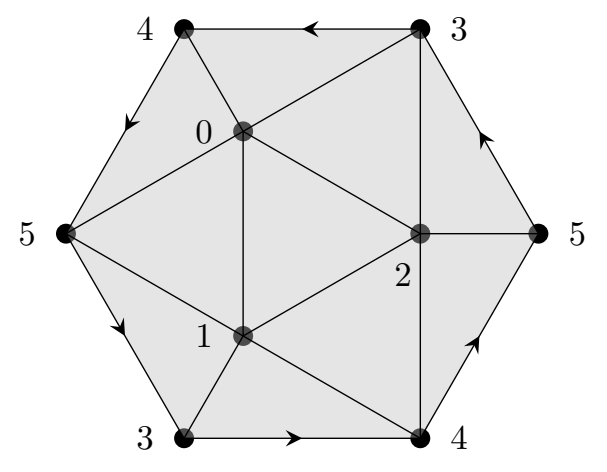

Figure 10: A 6-vertex triangulation of $\mathbb{R} P^{2}$.

We also extend Construction 3.5 by allowing more general star deletions in order to construct more non-trivial Massey products. These often only require a difference in the technical details of the proof of Theorem 3.17 and do not change the nature of the construction. For example, if $\mathcal{K}^{i}$ is the disjoint union of two vertices $\{i\}$ and $\left\{i^{\prime}\right\}$, then let $\mathcal{K}$ be the simplicial complex that is obtained from $\mathcal{K}^{1} * \mathcal{K}^{2} * \mathcal{K}^{3} * \mathcal{K}^{4}$ by the sequence of star deletions

$\operatorname{sd}_{\{1,4\}} \operatorname{sd}_{\left\{1,4^{\prime}\right\}} \operatorname{sd}_{\left\{1^{\prime}, 4^{\prime}\right\}} \operatorname{sd}_{\{2,4\}} \operatorname{sd}_{\left\{2^{\prime}, 4^{\prime}\right\}} \operatorname{sd}_{\left\{2,4^{\prime}\right\}} \operatorname{sd}_{\{1,3\}} \operatorname{sd}_{\left\{1^{\prime}, 3^{\prime}\right\}} \operatorname{sd}_{\left\{1,3^{\prime}\right\}} \operatorname{sd}_{\left\{3,4^{\prime}\right\}} \operatorname{sd}_{\left\{2,3^{\prime}\right\}} \operatorname{sd}_{\left\{1,2^{\prime}\right\}}$.

This is a full subcomplex of the icosahedron $\mathcal{I}$ as shown in [3, Theorem 4.6]. Also, no obstruction graph from the classification $[12,15]$ is a full subcomplex of $\mathcal{I}$. In [3], this example is given in order to demonstrate a non-trivial 4-Massey product of lowest-degree classes in $H^{*}\left(\mathcal{Z}_{\mathcal{I}}\right)$ where there are no non-trivial 3Massey products of lowest-degree classes in $H^{*}\left(\mathcal{Z}_{\mathcal{I}}\right)$.

Let us consider another example of more general star deletions. Suppose we have a simplicial complex $\mathcal{K}$ with $n \geqslant 3$ disjoint subsets of its vertices $J_{1}, \ldots, J_{n} \subset V(\mathcal{K})$ such that there are $n$ non-trivial classes $\alpha_{i} \in \widetilde{H}^{p_{i}}\left(\mathcal{K}_{J_{i}}\right)$. Limonchenko [20, Lemma 3.3] showed that if a Massey product $\left\langle\alpha_{1}, \ldots, \alpha_{n}\right\rangle \subset$ $H^{*}\left(\mathcal{Z}_{\mathcal{K}}\right)$ is defined and $\widetilde{H}^{p_{i}+\cdots+p_{k}}\left(\mathcal{K}_{J_{i} \cup \cdots \cup J_{k}}\right)=0$ for every $1 \leqslant i<k \leqslant n$, $(i, k) \neq(1, n)$, then the Massey product has trivial indeterminacy. The following example shows that this is not a necessary condition for trivial indeterminacy.

Example 3.22. For $i=1,2,3,4$, let $\mathcal{K}^{i}$ be the disjoint union of two vertices $J_{i}=\left\{i, i^{\prime}\right\}$. Let $\mathcal{K}$ be a simplicial complex obtained by Construction 3.5 with an additional star deletion at the edge $\left\{1^{\prime}, 2^{\prime}\right\}$, that is,

$$
\mathcal{K}=\operatorname{sd}_{\left\{2,4^{\prime}\right\}} \operatorname{sd}_{\left\{1,3^{\prime}\right\}} \operatorname{sd}_{\left\{3,4^{\prime}\right\}} \operatorname{sd}_{\left\{2,3^{\prime}\right\}} \operatorname{sd}_{\left\{1^{\prime}, 2^{\prime}\right\}} \operatorname{sd}_{\left\{1,2^{\prime}\right\}} \mathcal{K}^{1} * \mathcal{K}^{2} * \mathcal{K}^{3} * \mathcal{K}^{4} .
$$

For each $1 \leqslant i \leqslant 4$, let $a_{i}=\chi_{i} \in C^{0}\left(\mathcal{K}_{J_{i}}\right)$ and set $\alpha_{i}=\left[a_{i}\right] \in \widetilde{H}^{0}\left(\mathcal{K}_{J_{i}}\right)$. The star deletions at $\left\{1,2^{\prime}\right\}$ and $\left\{1^{\prime}, 2^{\prime}\right\}$ imply that any cochain $a_{1,2} \in C^{0}\left(\mathcal{K}_{J_{1} \cup J_{2}}\right)$ 
such that $d\left(a_{1,2}\right)=\overline{\overline{a_{1}}} a_{2}$ is of the form

$$
a_{1,2}=-\chi_{1}+c_{1}\left(\chi_{1}+\chi_{1^{\prime}}+\chi_{2}\right)+c_{1}^{\prime} \chi_{2^{\prime}}
$$

for any $c_{1}, c_{1}^{\prime} \in \mathbf{k}$. However, a cochain $a_{1,3}$ such that $d\left(a_{1,3}\right)=\overline{\overline{a_{1}}} a_{2,3}+\overline{\overline{a_{1,2}}} a_{3}$ is only defined when $c_{1}^{\prime}=c_{1}$. Thus, any defining system for $\left\langle\alpha_{1}, \alpha_{2}, \alpha_{3}, \alpha_{4}\right\rangle \subset$ $H^{*}\left(\mathcal{Z}_{\mathcal{K}}\right)$ is of the form

$$
\begin{aligned}
& a_{1,2}=-\chi_{1}+c_{1}\left(\chi_{1}+\chi_{1^{\prime}}+\chi_{2}+\chi_{2^{\prime}}\right) \\
& a_{2,3}=-\chi_{2}+c_{2}\left(\chi_{2}+\chi_{2^{\prime}}+\chi_{3}+\chi_{3^{\prime}}\right) \\
& a_{3,4}=-\chi_{3}+c_{3}\left(\chi_{3}+\chi_{3^{\prime}}+\chi_{4}+\chi_{4^{\prime}}\right) \\
& a_{1,3}=-\left(c_{2}-1\right) \chi_{1}+c_{1} \chi_{3}+c_{4}\left(\chi_{1}+\chi_{1^{\prime}}+\chi_{2}+\chi_{2^{\prime}}+\chi_{3}+\chi_{3^{\prime}}\right) \\
& a_{2,4}=-\left(c_{3}-1\right) \chi_{2}+c_{2} \chi_{4}+c_{5}\left(\chi_{2}+\chi_{2^{\prime}}+\chi_{3}+\chi_{3^{\prime}}+\chi_{4}+\chi_{4^{\prime}}\right)
\end{aligned}
$$

for coefficients $c_{1}, \ldots, c_{5} \in \mathbf{k}$. These are the same defining systems we would get if we had not star deleted $\mathcal{K}^{1} * \mathcal{K}^{2} * \mathcal{K}^{3} * \mathcal{K}^{4}$ at the edge $\left\{1^{\prime}, 2^{\prime}\right\}$. The associated cocycle $\omega$ to any of these defining systems is

$$
\begin{aligned}
\omega=-\chi_{14^{\prime}}-d\left(\chi_{1}\right)+c_{3} d\left(\chi_{1}\right)- & c_{1} d\left(\chi_{3}\right)+c_{4} d\left(\chi_{4}\right)-c_{5} d\left(\chi_{1}\right)+ \\
& +c_{1} c_{3}\left(-d\left(\chi_{1}\right)-d\left(\chi_{1^{\prime}}\right)-d\left(\chi_{2}\right)-d\left(\chi_{2^{\prime}}\right)\right) .
\end{aligned}
$$

Thus, $\left\langle\alpha_{1}, \alpha_{2}, \alpha_{3}, \alpha_{4}\right\rangle=[\omega]=\left[-\chi_{14^{\prime}}\right]$ and hence this Massey product is nontrivial and has no indeterminacy. However, the star deletions at $\left\{1,2^{\prime}\right\}$ and $\left\{1^{\prime}, 2^{\prime}\right\}$ imply that $\widetilde{H}^{0}\left(\mathcal{K}_{J_{1} \cup J_{2}}\right)=\mathbb{Z} \neq 0$. Therefore this is an example of a non-trivial Massey product with trivial indeterminacy that does not satisfy the conditions of [20, Lemma 3.3].

\subsection{Infinite families of Massey products with non-trivial indeterminacy}

In the last example, we saw that doing Construction 3.5 followed by an extra star deletion at $\left\{1^{\prime}, 2^{\prime}\right\}$ produced more choices of cochains $a_{1,2}$ such that $d\left(a_{1,2}\right)=\overline{\overline{a_{1}}} a_{2}$. We extend this technique to create the first infinite families of moment-angle complexes with non-trivial Massey products that have non-trivial indeterminacy. These are the first known examples of non-trivial indeterminacy in $n$-Massey products in $H^{*}\left(\mathcal{Z}_{\mathcal{K}}\right)$ for $n \geqslant 4$.

The idea in Construction 3.5 was to create a non-trivial Massey product $\left\langle\alpha_{1}, \ldots, \alpha_{n}\right\rangle$ by defining two sets of simplices $S_{a_{i}}, P_{a_{i}}$ for each $1 \leqslant i \leqslant n$ and star deleting the join of $n$ simplicial complexes at the simplices $\sigma_{i} \cup \sigma_{k}^{\prime}$ for $\sigma_{i} \in S_{a_{i}}$, $\sigma_{k}^{\prime} \in P_{a_{k}}, 1 \leqslant i<k \leqslant n,(i, k) \neq(1, n)$. A star deletion at $\sigma_{i} \cup \sigma_{k}^{\prime}$ made the Massey product $\left\langle\alpha_{i}, \ldots, \alpha_{k}\right\rangle$ trivial by allowing us to define a cochain $a_{i, k}$ such that $d\left(a_{i, k}\right)$ represents a (trivial) class in the lower Massey product $\left\langle\alpha_{i}, \ldots, \alpha_{k}\right\rangle$. Supposing that $\left\langle\alpha_{1}, \ldots, \alpha_{k}\right\rangle$ has non-trivial indeterminacy, we construct indeterminacy in the higher Massey product $\left\langle\alpha_{1}, \ldots, \alpha_{n}\right\rangle$ by making more than one class in the lower product $\left\langle\alpha_{1}, \ldots, \alpha_{k}\right\rangle$ trivial. In this version of the construction, we star delete at $\sigma_{1} \cup \sigma_{k}^{\prime}$ for $\sigma_{k}^{\prime} \in P_{a_{k}}, k \neq n$, and any $p_{1}$-simplex $\sigma_{1} \in \mathcal{K}^{1}$, rather than $\sigma_{1} \in S_{a_{1}} \subset \mathcal{K}^{1}$. These extra star deletions create choices for $a_{1, k}$ 
in the defining system for $\left\langle\alpha_{1}, \ldots, \alpha_{n}\right\rangle$, and do not affect the proof of Theorem 3.17. We will show that these choices result in non-trivial indeterminacy in $\left\langle\alpha_{1}, \ldots, \alpha_{n}\right\rangle$ when $n>2$.

Theorem 3.23. Let $\mathcal{K}^{i}$ be a simplicial complex on the vertex set $\left[m_{i}\right]$ that is not an $\left(m_{i}-1\right)$-simplex, for $i \in\{1, \ldots, n\}, n>2$. Then there exists a simplicial complex $\mathcal{K}$ obtained by star deletions on $\mathcal{K}^{1} * \cdots * \mathcal{K}^{n}$ such that $H^{*}\left(\mathcal{Z}_{\mathcal{K}}\right)$ has a non-trivial $n$-Massey product with non-trivial indeterminacy.

Proof. Since $\mathcal{K}^{i}$ is not an $\left(m_{i}-1\right)$-simplex, there is a non-trivial class $\alpha_{i} \in$ $\widetilde{H}^{p_{i}}\left(\mathcal{K}_{J_{i}}^{i}\right)$ for $J_{i} \subset\left[m_{i}\right]$. We will construct two different defining systems for a Massey product $\left\langle\alpha_{1}, \ldots, \alpha_{n}\right\rangle$ and show that the two associated cocycles are non-zero and not cohomologous. Therefore this concludes there is non-trivial indeterminacy in $\left\langle\alpha_{1}, \ldots, \alpha_{n}\right\rangle$.

Let $a_{i}$ be a cocycle representative for $\alpha_{i}$. Recall that in Construction 3.5, we had a set of $p_{i}$-simplices $S_{a_{i}} \subset \mathcal{K}^{i}$ for each $i$ such that $a_{i}=\sum_{\sigma_{i} \in S_{a_{i}}} c_{\sigma_{i}} \chi_{\sigma_{i}}$. For any $\sigma_{i} \in S_{a_{i}}$, the set $P_{\sigma_{i}} \subset \mathcal{K}^{i}$ contains all $p_{i}$-simplices $\sigma_{i}^{\prime} \in \mathcal{K}^{i}$ such that there is a vertex $v_{\sigma_{i}^{\prime}}$ and $\sigma_{i} \backslash v_{\sigma_{i}}=\sigma_{i}^{\prime} \backslash v_{\sigma_{i}^{\prime}}$, where $v_{\sigma_{i}}$ is a fixed choice of vertex in $\sigma_{i}$. We will use these fixed choices of $v_{\sigma_{i}} \in \sigma_{i} \in S_{a_{i}}$ and $v_{\sigma_{i}^{\prime}} \in \sigma_{i}^{\prime} \in P_{\sigma_{i}}$ throughout this proof. Also recall the set

$$
P_{a_{i}}=P_{\sigma_{i}^{(1)}} \cup \cdots \cup P_{\sigma_{i}^{(l)}}
$$

for $\sigma_{i}^{(1)}, \ldots, \sigma_{i}^{(l)} \subset S_{a_{i}}$. To define a simplicial complex $\mathcal{K}$ so that $\left\langle\alpha_{1}, \ldots, \alpha_{n}\right\rangle \subset$ $H^{*}\left(\mathcal{Z}_{\mathcal{K}}\right)$ has non-trivial indeterminacy, we star delete $\mathcal{K}^{1} * \cdots * \mathcal{K}^{n}$ at $\sigma_{1} \cup \sigma_{k}^{\prime}$ for every $p_{1}$-simplex $\sigma_{1} \in \mathcal{K}^{1}$ and $\sigma_{k}^{\prime} \in P_{a_{k}}, 1<k<n$, as well as at each $\sigma_{i} \cup \sigma_{k}^{\prime}$ for $\sigma_{i} \in S_{a_{i}}$ and $\sigma_{k}^{\prime} \in P_{a_{k}}, 1<i<k \leq n$. This is more star deletions than in Construction 3.5, where we used $\sigma_{1} \in S_{a_{1}}$ instead of $\sigma_{1} \in \mathcal{K}^{1}$. Let $\widetilde{S}_{a_{k}}=S_{a_{k}} \backslash P_{a_{k}}$. If there are simplices $\sigma_{k} \in S_{a_{k}} \backslash \widetilde{S}_{a_{k}}$ for any $k$, then we also star delete at $\sigma_{i}^{\prime} \cup \sigma_{k}$ for every $\sigma_{i}^{\prime} \in P_{a_{i}}, i<k$. This is for technical purposes, to ensure that $\sigma_{k} \in S_{a_{k}}$ and $\sigma_{i}^{\prime} \cup \sigma_{k} \in \mathcal{K}$ implies that $\sigma_{k} \in \widetilde{S}_{a_{k}}$.

We construct two different defining systems for $\left\langle\alpha_{1}, \ldots, \alpha_{n}\right\rangle$. Recall from (3.2) in Proposition 3.12 that $a_{i, k} \in C^{p_{i}+\cdots+p_{k}}\left(\mathcal{K}_{J_{i} \cup \cdots \cup J_{k}}\right)$ for $1 \leqslant i \leqslant k \leqslant n$, $(i, k) \neq(1, n)$ is the cochain

$$
a_{i, k}=\sum_{\sigma_{i} \in S_{a_{i}}} \sum_{\sigma_{i+1} \in \widetilde{S}_{a_{i+1}}} \cdots \sum_{\sigma_{k} \in \widetilde{S}_{a_{k}}} c_{\sigma_{i}} \ldots c_{\sigma_{k}} \theta_{i, k} \chi_{\sigma_{i} \cup \cdots \cup \sigma_{k} \backslash\left(v_{i+1} \cup \cdots \cup v_{k}\right)}
$$

where $\widetilde{S}_{a_{i}}=S_{a_{i}} \backslash P_{a_{i}}$ and $\theta_{i, k}=1$ when $i=k$ or otherwise

$$
\begin{aligned}
\theta_{i, k}=(-1)^{k-i+\left|J_{i}\right|\left(p_{i+1}+\cdots+p_{k}\right)+\left|J_{i+1}\right|\left(p_{i+2}+\cdots+p_{k}\right)+\cdots+\left|J_{k-1}\right| p_{k}} \\
\cdot \varepsilon\left(v_{\sigma_{i+1}}, \sigma_{i+1}\right) \ldots \varepsilon\left(v_{\sigma_{k}}, \sigma_{k}\right) .
\end{aligned}
$$

The defining system $\left(a_{i, k}\right)$ is a defining system for $\left\langle\alpha_{1}, \ldots, \alpha_{n}\right\rangle$ by the same proof as for Proposition 3.12, since neither the simplices $\sigma_{1} \cup \sigma_{k}$ for $\sigma_{1} \notin S_{a_{1}}$ and $\sigma_{k} \in P_{a_{k}}, 1<k<n$, nor $\sigma_{i}^{\prime} \cup \sigma_{k}$ for $\sigma_{i}^{\prime} \in P_{a_{i}}$ and $\sigma_{k} \in\left(S_{a_{k}} \backslash \widetilde{S}_{a_{k}}\right), i<k$, 
play an active role in the proof. To construct a different defining system, for any $1<k \leqslant n$, let $b_{1, k} \in C^{p_{1}+\cdots+p_{k}}\left(\mathcal{K}_{J_{1} \cup \cdots \cup J_{k}}\right)$ be the cochain

$$
b_{1, k}=\sum_{\sigma_{1} \in S_{a_{1}}} \sum_{\sigma_{2} \in \widetilde{S}_{a_{2}}} \cdots \sum_{\sigma_{k} \in \widetilde{S}_{a_{k}}} \sum_{\sigma_{i}^{\prime} \in P_{\sigma_{2}} \cup \cdots \cup P_{\sigma_{k}}} \varrho_{1, k} \chi_{v_{\sigma_{i}} \cup \sigma_{1} \cup \cdots \cup \sigma_{k} \backslash\left(v_{\sigma_{1}} \cup \cdots \cup v_{\sigma_{k}}\right)}
$$

where $\varrho_{1, k}=c_{\sigma_{1}} \ldots c_{\sigma_{k}} \varepsilon\left(v_{\sigma_{i}^{\prime}}, v_{\sigma_{i}^{\prime}} \cup \sigma_{1} \cup \cdots \cup \sigma_{k} \backslash\left(v_{\sigma_{1}} \cup \cdots \cup v_{\sigma_{k}}\right)\right) \theta_{1, k}$. Also let $b_{i, k}=0$ for $i \neq 1$ or $i=k=1$, so $a_{i, k}^{\prime}=a_{i, k}+b_{i, k}$ for all $1 \leqslant i \leqslant k \leqslant n$, $(i, k) \neq(1, n)$. We will show that $\left(a_{i, k}^{\prime}\right)$ is a defining system for $\left\langle\alpha_{1}, \ldots, \alpha_{n}\right\rangle$.

First we check that $d\left(b_{1, k}\right)=\sum_{r=1}^{k-1} \overline{\overline{b_{1, r}}} a_{r+1, k}$, where

$$
\begin{aligned}
& d\left(b_{1, k}\right)=\sum_{\sigma_{1} \in S_{a_{1}}} \sum_{\sigma_{2} \in \widetilde{S}_{a_{2}}} \cdots \sum_{\sigma_{k} \in \widetilde{S}_{a_{k}}} \sum_{\sigma_{i}^{\prime} \in P_{\sigma_{2}} \cup \cdots \cup P_{\sigma_{k}}} \sum_{j \in \mathcal{K}_{J_{1} \cup \cdots \cup J_{n}}} \varrho_{1, k} . \\
& \cdot \varepsilon\left(j, j \cup v_{\sigma_{i}^{\prime}} \cup \sigma_{1} \cup \cdots \cup \sigma_{k} \backslash\left(v_{\sigma_{1}} \cup \cdots \cup v_{\sigma_{k}}\right)\right) \\
& \chi_{j \cup v_{\sigma_{i}} \cup \sigma_{1} \cup \cdots \cup \sigma_{k} \backslash\left(v_{\sigma_{1}} \cup \cdots \cup v_{\sigma_{k}}\right)} .
\end{aligned}
$$

Fix a simplex $\tau=v_{\sigma_{i}^{\prime}} \cup \sigma_{1} \cup \cdots \cup \sigma_{k} \backslash\left(v_{\sigma_{1}} \cup \cdots \cup v_{\sigma_{k}}\right) \in S_{b_{1, k}}$. For any $1 \leqslant r \leqslant k$, recall from the definition of $P_{\sigma_{r}}$ that since $\sigma_{r} \in S_{a_{r}}$, if there is a vertex $v \in \mathcal{K}^{r}$ such that $v \cup\left(\sigma_{r} \backslash v_{\sigma_{r}}\right) \in \mathcal{K}^{r}$, then $v \cup\left(\sigma_{r} \backslash v_{\sigma_{r}}\right) \in P_{\sigma_{r}}$. Thus $\sigma_{i}^{\prime}=v_{\sigma_{i}^{\prime}} \cup\left(\sigma_{i} \backslash v_{\sigma_{i}}\right) \in P_{\sigma_{i}}$. Consider the link of $\tau$ in $\mathcal{K}_{J_{1} \cup \cdots \cup J_{k}}$. There is no vertex $v \in \mathcal{K}^{1}$ in this link since if $v \cup \sigma_{1} \backslash v_{\sigma_{1}} \in \mathcal{K}^{1}$, then $\left(v \cup \sigma_{1} \backslash v_{\sigma_{1}}\right) \cup \sigma_{i}^{\prime} \notin \mathcal{K}$ because there was a star deletion at that simplex. Similarly, for any $r<i$, there is no vertex $v_{\sigma_{r}}$ in the link of $\tau$ because $\sigma_{r} \cup \sigma_{i}^{\prime} \notin \mathcal{K}$. Therefore the only vertices in the link of $\tau$ are $v_{\sigma_{r}^{\prime}}$ for $\sigma_{r^{\prime}} \in P_{\sigma_{r}}$ and any $r$, and $v_{\sigma_{r}}$ for $\sigma_{r} \in S_{a_{r}}$ and $r>i$.

Consider the summands of $d\left(b_{1, k}\right)$ when $j=v_{\sigma_{r}^{\prime}}$ for $\sigma_{r^{\prime}} \in P_{\sigma_{r}}$ and any $r$. If $v_{\sigma_{i}^{\prime}} \cup v_{\sigma_{r}^{\prime}} \cup \sigma_{1} \cup \cdots \cup \sigma_{k} \backslash\left(v_{\sigma_{1}} \cup \cdots \cup v_{\sigma_{k}}\right) \in \mathcal{K}$, then the coefficient of $\chi_{v_{\sigma_{i}^{\prime}} \cup v_{\sigma_{r}^{\prime}} \cup \sigma_{1} \cup \cdots \cup \sigma_{k} \backslash\left(v_{\sigma_{1}} \cup \cdots \cup v_{\sigma_{k}}\right)}$ is the product of $c_{\sigma_{1}} \ldots c_{\sigma_{k}} \theta_{1, k}$ and

$$
\begin{gathered}
\varepsilon\left(v_{\sigma_{i}^{\prime}}, v_{\sigma_{i}^{\prime}} \cup \sigma_{1} \cup \cdots \cup \sigma_{k} \backslash\left(v_{\sigma_{1}} \cup \cdots \cup v_{\sigma_{k}}\right)\right) \varepsilon\left(v_{\sigma_{r}^{\prime}}, v_{\sigma_{i}^{\prime}} \cup v_{\sigma_{r}^{\prime}} \cup \sigma_{1} \cup \cdots \cup \sigma_{k} \backslash\left(v_{\sigma_{1}} \cup \cdots \cup v_{\sigma_{k}}\right)\right)+ \\
\varepsilon\left(v_{\sigma_{r}^{\prime}}, v_{\sigma_{r}^{\prime}} \cup \sigma_{1} \cup \cdots \cup \sigma_{k} \backslash\left(v_{\sigma_{1}} \cup \cdots \cup v_{\sigma_{k}}\right)\right) \varepsilon\left(v_{\sigma_{i}^{\prime}}, v_{\sigma_{i}^{\prime}} \cup v_{\sigma_{r}^{\prime}} \cup \sigma_{1} \cup \cdots \cup \sigma_{k} \backslash\left(v_{\sigma_{1}} \cup \cdots \cup v_{\sigma_{k}}\right)\right) .
\end{gathered}
$$

First suppose that $\sigma_{i}^{\prime}, \sigma_{r}^{\prime} \in \mathcal{K}^{i}$, so $\sigma_{i}^{\prime} \cup v_{\sigma_{r}^{\prime}}=\sigma_{r}^{\prime} \cup v_{\sigma_{i}^{\prime}} \in \mathcal{K}^{i}$. Also suppose, without loss of generality, that $v_{\sigma_{i}^{\prime}}<v_{\sigma_{r}^{\prime}}$ in the order of the vertex set of $\mathcal{K}$ and that $v_{\sigma_{r}^{\prime}}$ is the $l$ th vertex in $\sigma_{r}^{\prime}$. Since $v_{\sigma_{i}^{\prime}}<v_{\sigma_{r}^{\prime}}$ and $\varepsilon\left(v_{\sigma_{i}^{\prime}}, v_{\sigma_{i}^{\prime}} \cup \sigma_{1} \cup \cdots \cup \sigma_{k} \backslash\left(v_{\sigma_{1}} \cup \cdots \cup v_{\sigma_{k}}\right)\right)=$ $(-1)^{p_{1}+\cdots+p_{i-1}} \varepsilon\left(v_{\sigma_{i}^{\prime}}, \sigma_{i}^{\prime}\right)$ by the definition of $\varepsilon$ in (2.2), we rewrite (3.13) as

$$
\begin{aligned}
& \varepsilon\left(v_{\sigma_{i}^{\prime}}, \sigma_{i}^{\prime}\right) \varepsilon\left(v_{\sigma_{r}^{\prime}}, \sigma_{i}^{\prime} \cup v_{\sigma_{r}^{\prime}}\right)+\varepsilon\left(v_{\sigma_{r}^{\prime}}, \sigma_{r}^{\prime}\right) \varepsilon\left(v_{\sigma_{i}^{\prime}}, \sigma_{i}^{\prime} \cup v_{\sigma_{r}^{\prime}}\right)= \\
&(-1)^{l} \varepsilon\left(v_{\sigma_{i}^{\prime}}, \sigma_{i}^{\prime}\right)-(-1)^{l-1} \varepsilon\left(v_{\sigma_{i}^{\prime}}, \sigma_{i}^{\prime} \cup v_{\sigma_{r}^{\prime}}\right) .
\end{aligned}
$$

Also $\varepsilon\left(v_{\sigma_{i}^{\prime}}, \sigma_{i}^{\prime}\right)=\varepsilon\left(v_{\sigma_{i}^{\prime}}, \sigma_{i}^{\prime} \cup v_{\sigma_{r}^{\prime}}\right)$ because $v_{\sigma_{i}^{\prime}}<v_{\sigma_{r}^{\prime}}$, so (3.13) is zero. In particular, when $k=i=2$, then $d\left(b_{1,2}\right)=0$. So $d\left(a_{1,2}^{\prime}\right)=d\left(a_{1,2}\right)=\overline{\overline{a_{1}}} a_{2}$.

Alternatively, suppose that $\sigma_{i}^{\prime} \in \mathcal{K}^{i}, \sigma_{r}^{\prime} \in \mathcal{K}^{r}$ and, without loss of generality, $i<r$. By using the definition of $\varepsilon$ in (2.2), then (3.13) becomes

$$
\begin{aligned}
&(-1)^{p_{1}+\cdots+p_{i-1}} \varepsilon\left(v_{\sigma_{i}^{\prime}}, \sigma_{i}^{\prime}\right)(-1)^{p_{1}+\cdots+p_{r-1}+1} \varepsilon\left(v_{\sigma_{r}^{\prime}}, \sigma_{r}^{\prime}\right)+ \\
&(-1)^{p_{1}+\cdots+p_{r-1}} \varepsilon\left(v_{\sigma_{r}^{\prime}}, \sigma_{r}^{\prime}\right)(-1)^{p_{1}+\cdots+p_{i-1}} \varepsilon\left(v_{\sigma_{i}^{\prime}}, \sigma_{i}^{\prime}\right)=0 .
\end{aligned}
$$


Since all of these summands cancel out, we conclude that $d\left(b_{1, k}\right)$ only has non-zero summands when $j=v_{\sigma_{r}}$ for $\sigma_{r} \in S_{a_{r}}$ and $r>i$. By rewriting $r$ as $r+1$ for $r \in\{1, \ldots, n\}$ and $i \in\{2, \ldots, r\}, d\left(b_{1, k}\right)$ is equal to

$$
\begin{gathered}
\sum_{\sigma_{1} \in S_{a_{1}}} \sum_{\sigma_{2} \in \widetilde{S}_{a_{2}}} \cdots \sum_{\sigma_{k} \in \widetilde{S}_{a_{k}}} \sum_{r=1}^{k-1} \sum_{\sigma_{i}^{\prime} \in P_{\sigma_{2}} \cup \cdots \cup P_{\sigma_{r}}} \varrho_{i, k} \cdot \\
\cdot \varepsilon\left(v_{\sigma_{r+1}}, v_{\sigma_{i}^{\prime}} \cup v_{\sigma_{r+1}} \cup \sigma_{1} \cup \cdots \cup \sigma_{k} \backslash\left(v_{\sigma_{1}} \cup \cdots \cup v_{\sigma_{k}}\right)\right) \\
\chi_{v_{\sigma_{i}^{\prime}} \cup v_{\sigma_{r+1}} \cup \sigma_{1} \cup \cdots \cup \sigma_{k} \backslash\left(v_{\sigma_{1}} \cup \cdots \cup v_{\sigma_{k}}\right)} .
\end{gathered}
$$

Since the simplices $\sigma_{i}^{\prime} \cup \sigma_{r}$ were star deleted for $\sigma_{i}^{\prime} \in P_{a_{i}}$ and $\sigma_{r} \in S_{a_{r}} \backslash \widetilde{S}_{a_{r}}$, this sum is the same whether we use $\sum_{\sigma_{r} \in \widetilde{S}_{a_{r}}}$ or $\sum_{\sigma_{r} \in S_{a_{r}}}$. Therefore we split this sum into products so that $d\left(b_{1, k}\right)=\sum_{r=1}^{k-1} \overline{\overline{b_{1, r}}} a_{r+1, k}$, by using the fact that

$$
\begin{aligned}
& \varrho_{1, k} \varepsilon\left(v_{\sigma_{r+1}}, v_{\sigma_{i}^{\prime}} \cup v_{\sigma_{r+1}} \cup \sigma_{1} \cup \cdots \cup \sigma_{k} \backslash\left(v_{\sigma_{1}} \cup \cdots \cup v_{\sigma_{k}}\right)\right)= \\
& (-1)^{p_{1}+\cdots+p_{r}+\left|J_{1} \cup \cdots \cup J_{r}\right|\left(p_{r+1}+\cdots+p_{k}\right)} \varrho_{1, r} c_{\sigma_{r+1}} \ldots c_{\sigma_{k}} \theta_{r+1, k} .
\end{aligned}
$$

Then

$$
\begin{aligned}
d\left(a_{i, k}^{\prime}\right) & =d\left(a_{i, k}\right)+d\left(b_{i, k}\right) \\
& =\sum_{r=1}^{k-1} \overline{\overline{a_{1, r}}} a_{r+1, k}+\sum_{r=1}^{k-1} \overline{\overline{b_{1, r}}} a_{r+1, k}=\sum_{r=1}^{k-1} \overline{\overline{a_{1, r}^{\prime}}} a_{r+1, k} .
\end{aligned}
$$

Hence $\left(a_{i, k}^{\prime}\right)$ is a defining system for $\left\langle\alpha_{1}, \ldots, \alpha_{n}\right\rangle$.

The associated cocycle for $\left(a_{i, k}^{\prime}\right)$ is

$$
\omega^{\prime}=\omega+\sum_{r=1}^{n-1} \overline{\overline{b_{1, r}}} a_{r+1, n}
$$

where $\omega$ is the associated cocycle for $\left(a_{i, k}\right)$. We show that the difference $\omega^{\prime}-\omega$ is not a coboundary by constructing a cycle $x^{\prime} \in C_{p_{1}+\ldots+p_{n}+1}\left(\mathcal{K}_{J_{1} \cup \ldots \cup J_{n}}\right)$ such that $\left(\omega^{\prime}-\omega\right)(x) \neq 0$. We use a similar method to Construction 3.13. Fix $\sigma_{i} \in \widetilde{S}_{a_{i}}$ for $i=$ $1,3, \ldots, n-1$ and fix $\sigma_{2}^{\prime} \in P_{a_{2}}$. Since it was star deleted, $\sigma_{1} \cup \sigma_{2}^{\prime} \notin \mathcal{K}$ but the boundary complex $\partial\left(\sigma_{1} \cup \sigma_{2}^{\prime}\right)$ is contained in $\mathcal{K}$. Also, since $\alpha_{n} \in \widetilde{H}^{p_{n}}\left(\mathcal{K}_{J_{n}}\right)$ is non-zero, there is a cycle $x_{n} \in C_{p_{n}}\left(\mathcal{K}_{J_{n}}\right)$ such that $a_{n}\left(x_{n}\right) \neq 0$. Define $x^{\prime} \in C_{p_{1}+\ldots+p_{n}+1}\left(\mathcal{K}_{J_{1} \cup \ldots \cup J_{n}}\right)$ to be the chain

$$
\begin{aligned}
x^{\prime}= & \sum_{w_{2} \in \sigma_{1} \cup \sigma_{2}^{\prime}} \sum_{w_{3} \in \sigma_{3}} \cdots \sum_{w_{n-1} \in \sigma_{n-1}} \\
& \sum_{\tilde{\sigma}_{n} \in S_{x_{n}}} c_{w_{2}} \cdots c_{w_{n-1}} c_{\tilde{\sigma}_{n}} \Delta_{\sigma_{1} \cup \sigma_{2}^{\prime} \cup \sigma_{3} \cdots \cup \sigma_{n-1} \cup \tilde{\sigma}_{n} \backslash\left(w_{2} \cup \cdots \cup w_{n-1}\right)}
\end{aligned}
$$

where $c_{\tilde{\sigma}_{n}}$ are the non-zero coefficients from $x_{n}$, and $c_{w_{2}}, \ldots, c_{w_{n-1}}$ are the coefficients of cycles in $C_{p_{1}+p_{2}}\left(\partial\left(\sigma_{1} \cup \sigma_{2}^{\prime}\right)\right), C_{p_{i}}\left(\partial\left(\sigma_{i}\right)\right)$ for $3 \leqslant i \leqslant n-1$. Every simplex $\sigma$ in the support $S_{x}$ of $x$ is a simplex in $\mathcal{K}$ since none of them were star deleted. By an analogous proof to Lemma 3.14, the chain $x^{\prime}$ is a cycle.

We want to compare the supports $S_{\omega^{\prime}-\omega}$ of $\omega^{\prime}-\omega$ and $S_{x^{\prime}}$ of $x^{\prime}$. The cochain $\omega^{\prime}-\omega=\sum_{r=1}^{n-1} \overline{\overline{b_{1, r}}} a_{r+1, n}$ is given in (3.14) when $k=n$. A simplex $\sigma$ is in $S_{\omega^{\prime}-\omega} \cap S_{x^{\prime}}$ 
precisely when $w_{2}=v_{\sigma_{1}}, w_{j}=v_{\sigma_{j}}$ for $3 \leqslant j \leqslant n-1, r=n-1$ so that $\sigma_{r+1}=\sigma_{n} \in S_{x_{n}}$, and $i=2 \in\{2, \ldots, r\}$ so that $\sigma_{i}^{\prime}=\sigma_{2}^{\prime} \in P_{a_{2}}$. Hence $S_{\omega^{\prime}-\omega} \cap S_{x^{\prime}}$ contains only one simplex, $\sigma$. Thus $\left(\omega^{\prime}-\omega\right)(x)= \pm \chi_{\sigma}\left(\Delta_{\sigma}\right) \neq 0$. Therefore $\omega^{\prime}-\omega$ is not a coboundary and so $\left[\omega^{\prime}\right] \neq[\omega]$.

The proof that $\left\langle\alpha_{1}, \ldots, \alpha_{n}\right\rangle$ is non-trivial is the same as the proof of Proposition 3.16 since neither the simplices $\sigma_{1} \cup \sigma_{k}^{\prime}$ for $\sigma_{1} \notin S_{a_{1}}$ and $\sigma_{k}^{\prime} \in P_{a_{k}}$, nor $\sigma_{i}^{\prime} \cup \sigma_{k}$ for $\sigma_{i} \in P_{a_{i}}$ and $\sigma_{k} \in\left(S_{a_{k}} \backslash \widetilde{S}_{a_{k}}\right), i<k$, play a role so the extra star deletions do not change the proof. Hence $\left\langle\alpha_{1}, \ldots, \alpha_{n}\right\rangle$ is non-trivial with non-trivial indeterminacy.

Example 3.24. For $i=1,2,3$, suppose $\mathcal{K}^{i}$ is a pair of disjoint vertices labelled $\sigma_{i}, \sigma_{i}^{\prime}$. Let $\alpha_{i} \in \widetilde{H}^{0}\left(\mathcal{K}^{i}\right)$ be represented by the cocycle $a_{i}=\chi_{\sigma_{i}}$. Then $S_{a_{i}}=\left\{\sigma_{i}\right\}$ and $P_{a_{i}}=\left\{\sigma_{i}^{\prime}\right\}$. Following the construction in the proof of Theorem 3.23, we define

$$
\mathcal{K}=\operatorname{sd}_{\sigma_{1} \cup \sigma_{2}^{\prime}} \operatorname{sd}_{\sigma_{1}^{\prime} \cup \sigma_{2}^{\prime}} \operatorname{sd}_{\sigma_{2} \cup \sigma_{3}^{\prime}} \mathcal{K}^{1} * \mathcal{K}^{2} * \mathcal{K}^{3} .
$$

This simplicial complex is shown in Figure 11. The Massey product $\left\langle\alpha_{1}, \alpha_{2}, \alpha_{3}\right\rangle$ is one of the simplest examples of a Massey product in a moment-angle complex with non-trivial indeterminacy. It is one of the obstruction graphs in the classification of lowest degree non-trivial triple Massey products in [15]. Since it is a triple Massey product, its indeterminacy is given by $\alpha_{1} \cdot \widetilde{H}^{0}\left(\mathcal{K}_{\sigma_{2}, \sigma_{2}^{\prime}, \sigma_{3}, \sigma_{3}^{\prime}}\right)+\alpha_{3}$. $\widetilde{H}^{0}\left(\mathcal{K}_{\sigma_{1}, \sigma_{1}^{\prime}, \sigma_{2}, \sigma_{2}^{\prime}}\right)=\alpha_{3} \cdot \widetilde{H}^{0}\left(\mathcal{K}_{\sigma_{1}, \sigma_{1}^{\prime}, \sigma_{2}, \sigma_{2}^{\prime}}\right)$.

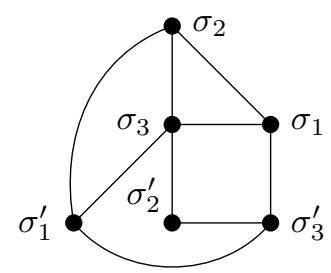

Figure 11: A simplicial complex $\mathcal{K}$ such that a triple Massey product in $H^{*}\left(\mathcal{Z}_{\mathcal{K}}\right)$ has indeterminacy.

\section{Massey products constructed by edge contractions}

A simplicial homotopy map $\varphi: \mathcal{K} \rightarrow \hat{\mathcal{K}}$ induces a map on the cohomology of moment-angle complexes $\varphi^{*}: H^{*}\left(\mathcal{Z}_{\hat{\mathcal{K}}}\right) \rightarrow H^{*}\left(\mathcal{Z}_{\mathcal{K}}\right)$. However, a property of Massey products $\left[17\right.$, Section 2] is that $\varphi^{*}\left\langle\hat{\alpha}_{1}, \ldots, \hat{\alpha}_{n}\right\rangle \subset\left\langle\varphi^{*}\left(\hat{\alpha}_{1}\right), \ldots, \varphi^{*}\left(\hat{\alpha}_{n}\right)\right\rangle$. Hence if $\left\langle\varphi^{*}\left(\hat{\alpha}_{1}\right), \ldots, \varphi^{*}\left(\hat{\alpha}_{n}\right)\right\rangle$ has non-trivial indeterminacy, it may be trivial even if $\left\langle\hat{\alpha}_{1}, \ldots, \hat{\alpha}_{n}\right\rangle$ is non-trivial.

In this section we use edge contractions $\varphi: \mathcal{K} \rightarrow \hat{\mathcal{K}}$ as a simplicial homotopy operation to construct non-trivial Massey products. Given a non-trivial Massey product $\left\langle\hat{\alpha}_{1}, \ldots, \hat{\alpha}_{n}\right\rangle \subset H^{*}\left(\mathcal{Z}_{\hat{\mathcal{K}}}\right)$ with $\hat{\alpha}_{i} \in \widetilde{H}^{p_{i}}\left(\hat{\mathcal{K}}_{\hat{J}_{i}}\right), \hat{J}_{i} \neq \hat{J}_{j}$ for $i \neq j$, we explicitly construct a defining system to show that $\left\langle\alpha_{1}, \ldots, \alpha_{n}\right\rangle \subset H^{*}\left(\mathcal{Z}_{\mathcal{K}}\right)$ is defined where $\alpha_{i}$ is the pullback of $\hat{\alpha}_{i}$ along $\varphi$. Then we also show that it is non-trivial to conclude the main result of this section, Theorem 4.12. 
Definition 4.1. Let $\mathcal{K}, \hat{\mathcal{K}}$ be simplicial complexes with an edge $\{u, w\} \in \mathcal{K}$, and a vertex $z \in V(\hat{\mathcal{K}})$ such that $V(\hat{\mathcal{K}}) \backslash\{z\}=V(\mathcal{K}) \backslash\{\{u\},\{w\}\}$. The simplicial complex $\hat{\mathcal{K}}$ is obtained from $\mathcal{K}$ by an edge contraction of $\{u, w\}$ if there is a map $\varphi_{V}: V(\mathcal{K}) \rightarrow V(\hat{\mathcal{K}})$

$$
\varphi_{V}(v)= \begin{cases}z & \text { for } v \in\{u, w\} \\ v & \text { for } v \notin\{u, w\}\end{cases}
$$

that extends to a surjective map $\varphi: \mathcal{K} \rightarrow \hat{\mathcal{K}}$, where $\varphi(I)=\left\{\varphi_{V}\left(v_{1}\right), \ldots, \varphi_{V}\left(v_{n}\right)\right\}$ for $I=\left\{v_{1}, \ldots, v_{n}\right\} \in \mathcal{K}$. The map $\varphi: \mathcal{K} \rightarrow \hat{\mathcal{K}}$ is called the edge contraction of $\{u, w\} \in \mathcal{K}$.

Edge contractions are simplicial maps, but they do not preserve the topology of $\mathcal{K}$ in general. Attali, Lieutier and Salinas [1, Theorem 2] showed that the homotopy type of a simplicial complex is preserved under edge contractions that satisfy the link condition.

Theorem $4.2([1])$. For any simplicial complex $\mathcal{K}$, if an edge $\{u, w\} \in \mathcal{K}$ satisfies the link condition,

$$
\operatorname{lk}_{\mathcal{K}}(\{u\}) \cap \operatorname{lk}_{\mathcal{K}}(\{w\})=\operatorname{lk}_{\mathcal{K}}(\{u, w\})
$$

then the edge contraction of $\{u, w\}$ preserves the homotopy type of $\mathcal{K}$.

Example 4.3. The following is a series of edge contractions that satisfy the link condition.

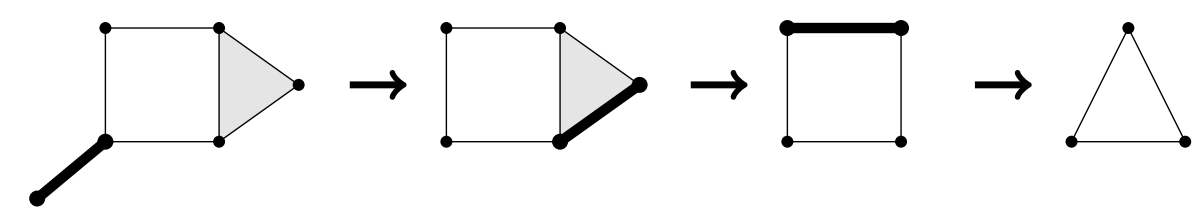

Example 4.4. Without the link condition, the homotopy type of a simplicial complex under edge contractions can change, such as in the following example.

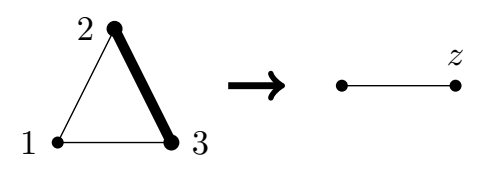

The links of the vertices $\{2\}$ and $\{3\}$ both contain the vertex $\{1\}$, but $\operatorname{lk}_{\mathcal{K}}(\{2,3\})$ is empty, so the link condition is not satisfied.

Example 4.5. An edge contraction that does not satisfy the link condition may create a non-trivial cycle. For example, suppose that $\hat{\mathcal{K}}$ is a triangulation of $S^{2}$ on four vertices, and let $\mathcal{K}$ be a 2-dimensional simplicial complex on 5 vertices with facets $\{1,2,3\},\{1,2,4\},\{1,3,4\},\{3,4,5\},\{2,5\}$ as shown in Figure 12. There is no non-trivial 2-cycle in $\mathcal{K}$ so $H^{2}(\mathcal{K})=0$, but the contraction $\{2,5\} \mapsto z$ results in a 2 -cycle and $H^{2}(\hat{\mathcal{K}}) \neq 0$. In this case the link condition is not satisfied because $\operatorname{lk}_{\mathcal{K}}\{2,5\}=\emptyset$ but $\operatorname{lk}_{\mathcal{K}}\{2\} \cap \mathrm{lk}_{\mathcal{K}}\{5\}=\{\{3\},\{4\}\}$. 


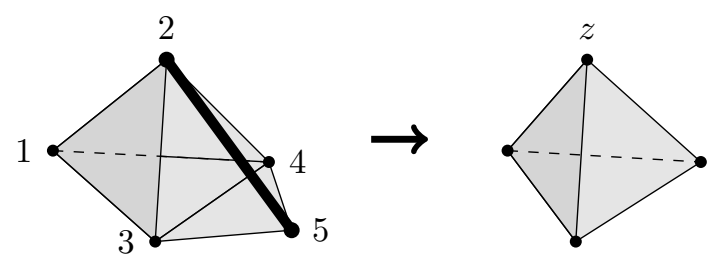

Figure 12

We construct cohomological classes in $H^{*}\left(\mathcal{Z}_{\mathcal{K}}\right)$ on which a new pulled-back Massey product will be defined.

Construction 4.6. Let $\hat{\mathcal{K}}$ be a simplicial complex with a non-trivial $n$-Massey product $\left\langle\hat{\alpha}_{1}, \ldots, \hat{\alpha}_{n}\right\rangle \subset H^{*}\left(\mathcal{Z}_{\hat{\mathcal{K}}}\right)$. By Hochster's theorem, every class $\hat{\alpha}_{i} \in$ $H^{*}\left(\mathcal{Z}_{\hat{\mathcal{K}}}\right)$ has a corresponding class

$$
\hat{\alpha}_{i} \in \widetilde{H}^{p_{i}}\left(\hat{\mathcal{K}}_{\hat{J}_{i}}\right)
$$

for a set of vertices $\hat{J}_{i} \subset V(\hat{\mathcal{K}})$. When $\left\langle\hat{\alpha}_{1}, \ldots, \hat{\alpha}_{n}\right\rangle$ is non-trivial, the sets of vertices $\hat{J}_{i}, \hat{J}_{j}$ are disjoint for any $i \neq j$.

Suppose that there is a simplicial complex $\mathcal{K}$ and a series of edge contractions $\varphi: \mathcal{K} \rightarrow \hat{\mathcal{K}}$ satisfying the link condition. Let the vertices in $V(\hat{\mathcal{K}})$ be ordered and suppose that all of the vertices in $\hat{J}_{i}$ come before those of $\hat{J}_{i+1}$. For a set of $p$-simplices $P \subset \hat{\mathcal{K}}$, let

$$
\varphi_{p}^{-1}(P)=\{\sigma \in \mathcal{K}|| \sigma \mid=p+1 \text { and } \varphi(\sigma)=\hat{\sigma} \text { for } \hat{\sigma} \in P\} .
$$

Suppose that the vertices $V(\mathcal{K})$ are ordered in such a way that for any vertex $\hat{v}$ that comes before $\hat{w}$ in $\hat{\mathcal{K}}$, each vertex $v \in \varphi_{0}^{-1}(\hat{v})$ comes before every $w \in$ $\varphi_{0}^{-1}(\hat{w})$. Let $J_{i}=\varphi_{0}^{-1}\left(\hat{J}_{i}\right) \subset V(\mathcal{K})$. Then by the order on $V(\mathcal{K})$, all vertices in $J_{i}$ come before those in $J_{i+1}$. Also $J_{i} \cap J_{j}=\emptyset$ for any $i \neq j$ since $\hat{J}_{i} \cap \hat{J}_{j}=\emptyset$ and $\varphi_{0}^{-1}(\hat{v}) \cap \varphi_{0}^{-1}(\hat{w})=\emptyset$ for any vertices $\hat{v}, \hat{w} \in \hat{\mathcal{K}}, \hat{v} \neq \hat{w}$.

Let $\hat{a}_{i}$ be a cocycle representing $\hat{\alpha}_{i} \in \widetilde{H}^{p_{i}}\left(\hat{\mathcal{K}}_{\hat{J}_{i}}\right)$. Let $S_{\hat{a}_{i}}$ be the support of $\hat{a}_{i}$, that is, the set of $p_{i}$-simplices $\hat{\sigma}_{i} \in \hat{\mathcal{K}}_{\hat{J}_{i}}$ such that

$$
\hat{a}_{i}=\sum_{\hat{\sigma} \in S_{\hat{a}_{i}}} c_{\hat{\sigma}} \chi_{\hat{\sigma}} \in C^{p_{i}}\left(\hat{\mathcal{K}}_{\hat{J}_{i}}\right)
$$

for non-zero coefficients $c_{\hat{\sigma}_{i}} \in \mathbf{k}$. Define $a_{i} \in C^{p_{i}}\left(\mathcal{K}_{J_{i}}\right)$ to be the cochain

$$
a_{i}=\sum_{\hat{\sigma} \in S_{\hat{a}_{i}}} c_{\hat{\sigma}} \sum_{\sigma \in \varphi_{p_{i}}^{-1}(\hat{\sigma})} \chi_{\sigma} .
$$

Since $a_{i}$ is a pullback of $\hat{a}_{i}$ along $\varphi, a_{i}$ is a cocycle and $\alpha_{i}=\left[a_{i}\right] \in \widetilde{H}^{p_{i}}\left(\mathcal{K}_{J_{i}}\right)$ is non-zero. 
Example 4.7. Let $\mathcal{K}_{J_{i}}, \hat{\mathcal{K}}_{\hat{J}_{i}}$ be the simplicial complexes as shown below, where $\hat{\mathcal{K}}_{\hat{J}_{i}}$ is obtained from $\mathcal{K}_{J_{i}}$ by contracting the edges $e_{2}=\{2,3\} \mapsto\{\hat{2}\}$ and $e_{5}=\{4,5\} \mapsto\{\hat{3}\}$. The cohomology class $\hat{\alpha}_{i} \in \widetilde{H}^{1}\left(\hat{\mathcal{K}}_{\hat{J}_{i}}\right)$ is represented by the cocycle $\chi_{\hat{e}}$, so let $S_{\hat{a}_{i}}=\{\hat{e}\}$.
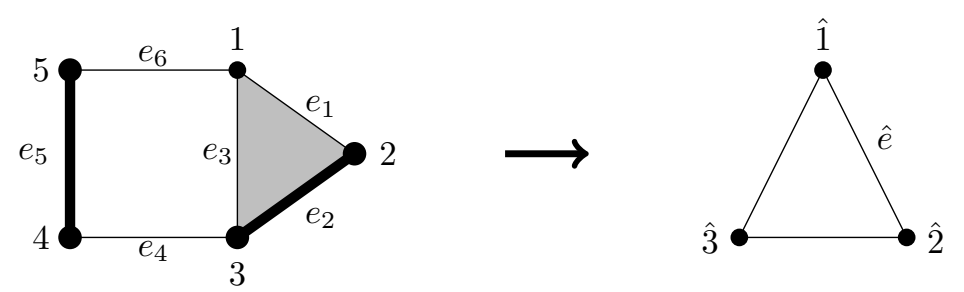

The contraction of $e_{2}$ satisfies the link condition, since $\operatorname{lk}_{\mathcal{K}}\left(e_{2}\right)=\operatorname{lk}_{\mathcal{K}}\{2\} \cap$ $\operatorname{lk}_{\mathcal{K}}\{3\}=\{1\}$. Under the map $\varphi: \mathcal{K} \rightarrow \hat{\mathcal{K}}, \varphi_{1}^{-1}(\hat{e})=\left\{e_{1}, e_{3}\right\}$. So by $(4.2), a_{i}$ is the cochain

$$
a_{i}=\chi_{e_{1}}+\chi_{e_{3}} \in C^{1}\left(\mathcal{K}_{J_{i}}\right) .
$$

This is a cocycle since $d\left(a_{i}\right)=\chi_{\{1,2,3\}}-\chi_{\{1,2,3\}}=0$.

For the Massey product $\left\langle\hat{\alpha}_{1}, \ldots, \hat{\alpha}_{n}\right\rangle \subset H^{\left(p_{1}+\cdots+p_{n}\right)+\left|\hat{J}_{1} \cup \cdots \cup \hat{J}_{n}\right|+2}\left(\mathcal{Z}_{\hat{\mathcal{K}}}\right)$, there is a defining system $\left(\hat{a}_{i, k}\right)$ for cochains $\hat{a}_{i, k} \in C^{p_{i}+\cdots+p_{k}}\left(\hat{\mathcal{K}}_{\hat{J}_{i} \cup \ldots \cup \hat{J}_{k}}\right), 1 \leqslant i \leqslant k \leqslant$ $n$ and $(i, k) \neq(1, n)$. Suppose that

$$
\hat{a}_{i, k}=\sum_{\hat{\tau} \in S_{\hat{a}_{i, k}}} c_{\hat{\tau}} \chi_{\hat{\tau}}
$$

for simplices $\hat{\tau} \in S_{\hat{a}_{i, k}} \subset \hat{\mathcal{K}}_{\hat{J}_{i} \cup \ldots \cup \hat{J}_{k}}$, non-zero coefficients $c_{\hat{\tau}} \in \mathbf{k}$. Then

$$
d\left(\hat{a}_{i, k}\right)=\sum_{\hat{\tau} \in S_{\hat{a}_{i, k}}} c_{\hat{\tau}}\left(\sum_{\hat{j} \in \hat{J}_{i} \cup \ldots \cup \hat{J}_{k} \backslash V(\hat{\tau})} \varepsilon(\hat{j}, \hat{j} \cup \hat{\tau}) \chi_{\hat{j} \cup \hat{\tau}}\right)
$$

is equal to

$$
\sum_{r=i}^{k-1}(-1)^{1+\overline{\operatorname{deg}}\left(\hat{a}_{i, r}\right)} \hat{a}_{i, r} \hat{a}_{r, k}=\sum_{r=i}^{k-1}(-1)^{1+\overline{\operatorname{deg}}\left(\hat{a}_{i, r}\right)} c\left(\sum_{\hat{\nu} \in S_{\hat{a}_{i, r}}} \sum_{\hat{\eta} \in S_{\hat{a}_{r+1}, k}} c_{\hat{\nu}} c_{\hat{\eta}} \chi_{\hat{\nu} \cup \hat{\eta}}\right)
$$

where $c=(-1)^{\left|\hat{J}_{i} \cup \cdots \cup \hat{J}_{r}\right|\left(p_{r+1}+\cdots+p_{k}+1\right)}$ comes from the product of $\hat{a}_{i, r}$ and $\hat{a}_{r, k}$, as in Lemma 2.2 , and $(-1)^{1+\overline{\operatorname{deg}}\left(\hat{a}_{i, r}\right)}=(-1)^{\left(p_{i}+\ldots+p_{r}\right)+\left|\hat{J}_{i} \cup \ldots \cup \hat{J}_{r}\right|}$. We use this defining system to construct a defining system for $\left\langle\alpha_{1}, \ldots, \alpha_{n}\right\rangle$.

Proposition 4.8. Let $\mathcal{K}$ be a simplicial complex that maps to $\hat{\mathcal{K}}$ by edge contractions satisfying the link condition. Then there is an $n$-Massey product $\left\langle\alpha_{1}, \ldots, \alpha_{n}\right\rangle$ defined on $H^{*}\left(\mathcal{Z}_{\mathcal{K}}\right)$. 
Proof. For every $i \in\{1, \ldots, n\}$, let $\alpha_{i}=\left[a_{i}\right]$ for $a_{i}$ as in (4.2). We start by constructing a defining system $\left(a_{i, k}\right)$ for $\left\langle\alpha_{1}, \ldots, \alpha_{n}\right\rangle \subset H^{*}\left(\mathcal{Z}_{\mathcal{K}}\right)$, where $a_{i, k} \in$ $C^{p_{i}+\cdots+p_{k}}\left(\mathcal{K}_{J_{i} \cup \cdots \cup J_{k}}\right)$. Define

$$
a_{i, k}=\theta_{i, k} \hat{\theta}_{i, k} \sum_{\hat{\tau} \in S_{\hat{a}_{i, k}}} c_{\hat{\tau}}\left(\sum_{\tau \in \varphi_{p_{i}+\cdots+p_{k}}^{-1}(\hat{\tau})} \chi_{\tau}\right)
$$

for $S_{\hat{a}_{i, k}}$ and $c_{\hat{\tau}} \in \mathbf{k}$ from (4.3), $\theta_{i, i}=1=\hat{\theta}_{i, i}$, and

$$
\begin{aligned}
& \theta_{i, k}=(-1)^{\left|J_{i}\right|\left(p_{i+1}+\cdots+p_{k}\right)}(-1)^{\left|J_{i+1}\right|\left(p_{i+2}+\cdots+p_{k}\right)} \cdots(-1)^{\left|J_{k-1}\right| p_{k}} \\
& \hat{\theta}_{i, k}=(-1)^{\left|\hat{J}_{i}\right|\left(p_{i+1}+\cdots+p_{k}\right)}(-1)^{\left|\hat{J}_{i+1}\right|\left(p_{i+2}+\cdots+p_{k}\right)} \cdots(-1)^{\left|\hat{J}_{k-1}\right| p_{k}} .
\end{aligned}
$$

Since $\theta_{i, i}=1=\hat{\theta}_{i, i}, a_{i, i}=a_{i}$ as in (4.2). We show that $d\left(a_{i, k}\right)=\sum_{r=i}^{k-1} \overline{\overline{a_{i, r}}} a_{r, k}$, where $\overline{\overline{a_{i, r}}}=(-1)^{1+\overline{\operatorname{deg}} a_{i, r}} a_{i, r}$ as in Definition 2.4.

Applying the coboundary map to $a_{i, k}, d\left(a_{i, k}\right)$ is

$$
\begin{aligned}
& \theta_{i, k} \hat{\theta}_{i, k} \sum_{\hat{\tau} \in S_{\hat{a}_{i, k}}} c_{\hat{\tau}}\left(\sum_{\tau \in \varphi_{p_{i}+\cdots+p_{k}}^{-1}(\hat{\tau})} \sum_{j \in J_{i} \cup \ldots \cup J_{k} \backslash V(\tau)} \varepsilon(j, j \cup \tau) \chi_{j \cup \tau}\right) \\
& =\theta_{i, k} \hat{\theta}_{i, k} \sum_{\hat{\tau} \in S_{\hat{a}_{i, k}}} c_{\hat{\tau}}\left(\sum_{\tau \in \varphi_{p_{i}+\cdots+p_{k}}^{-1}(\hat{\tau})} \sum_{j \in J_{i} \cup \cdots \cup J_{k} \backslash \varphi_{0}^{-1}(V(\hat{\tau}))} \varepsilon(j, j \cup \tau) \chi_{j \cup \tau}\right)+ \\
& +\theta_{i, k} \hat{\theta}_{i, k} \sum_{\hat{\tau} \in S_{\hat{a}_{i, k}}} c_{\hat{\tau}}\left(\sum_{\tau \in \varphi_{p_{i}+\cdots+p_{k}}^{-1}(\hat{\tau})} \sum_{j \in \varphi_{0}^{-1}(V(\hat{\tau})) \backslash V(\tau)} \varepsilon(j, j \cup \tau) \chi_{j \cup \tau}\right) .
\end{aligned}
$$

For any $\left(p_{i}+\cdots+p_{k}\right)$-simplex $\hat{\tau} \in S_{\hat{a}_{i, k}}$ and any $\tau \in \varphi_{p_{i}+\cdots+p_{k}}^{-1}(\hat{\tau})$, first suppose that there is a vertex $j \in \varphi_{0}^{-1}(V(\hat{\tau})) \backslash V(\tau)$ such that $j \cup \tau \in \mathcal{K}$. Then $j \cup \tau=$ $\bar{\tau} \in \varphi_{p_{i}+\cdots+p_{k}+1}^{-1}(\hat{\tau})$ and there is a vertex $i \in V(\tau)$ such that $\varphi(i)=\varphi(j)$. Thus $j \cup \tau \backslash i \in \varphi_{p_{i}+\cdots+p_{k}}^{-1}(\hat{\tau})$. Moreover, $i, j$ are consecutive vertices in $V(\bar{\tau})$ by the order of vertices in $\mathcal{K}$ defined in Construction 4.6, so $\varepsilon(j, \bar{\tau})=-\varepsilon(i, \bar{\tau})$. Therefore (4.8) is zero since all summands cancel out in pairs, that is, for any $\hat{\tau} \in S_{\hat{a}_{i, k}}$,

$$
\begin{aligned}
& \sum_{\tau \in \varphi_{p_{i}+\cdots+p_{k}}^{-1}(\hat{\tau})} \sum_{j \in \varphi_{0}^{-1}(V(\hat{\tau})) \backslash V(\tau)} \varepsilon(j, j \cup \tau) \chi_{j \cup \tau}= \\
& =\sum_{\substack{\bar{\tau} \in \varphi_{p_{i}+\cdots+p_{k}+1}^{-1}(\hat{\tau}), i, j \in \bar{\tau} \mid \varphi(i)=\varphi(j)}} \varepsilon(j, \bar{\tau}) \chi_{\bar{\tau}}+\varepsilon(i, \bar{\tau}) \chi_{\bar{\tau}}=0 .
\end{aligned}
$$


Consider summands in (4.7). For any $j \in J_{i} \cup \cdots \cup J_{k} \backslash \varphi_{0}^{-1}(V(\hat{\tau})), \varphi(j) \notin$ $V(\hat{j})$. So for any simplex $j \cup \tau \in \mathcal{K}$ with $j \in J_{i} \cup \cdots \cup J_{k} \backslash \varphi_{0}^{-1}(V(\hat{\tau}))$, there is a simplex $\varphi(j) \cup \hat{\tau} \in \hat{\mathcal{K}}$. Therefore any summand in (4.7) has a corresponding summand in the expression for $d\left(\hat{a}_{i, k}\right)$. Hence we rewrite (4.7) as

$$
d\left(a_{i, k}\right)=\theta_{i, k} \hat{\theta}_{i, k} \sum_{\hat{\tau} \in S_{\hat{a}_{i, k}}} c_{\hat{\tau}}\left(\sum_{\hat{j} \in \hat{J}_{i} \cup \ldots \cup \hat{J}_{k} \backslash V(\hat{\tau})} \sum_{j \cup \tau \in \varphi_{p_{i}+\cdots+p_{k}+1}^{-1}(\hat{j} \cup \hat{\tau})} \varepsilon(j, j \cup \tau) \chi_{j \cup \tau}\right)
$$

where, by the order of vertices in $\mathcal{K}, \varepsilon(j, j \cup \tau)=\varepsilon(\hat{j}, \hat{j} \cup \hat{\tau})$. Since $d\left(\hat{a}_{i, k}\right)=$ $\sum_{r=i}^{k-1} \overline{\hat{\hat{a}}_{i, r}} \hat{a}_{r, k}$, the expression in (4.9) can be written in terms of the expression in (4.4). Thus $d\left(a_{i, k}\right)$ is equal to

$$
\theta_{i, k} \hat{\theta}_{i, k} \sum_{r=i}^{k-1}(-1)^{1+\overline{\operatorname{deg}}\left(\hat{a}_{i, r}\right)} c\left(\sum_{\hat{\nu} \in S_{\hat{a}_{i, r}}} \sum_{\hat{\eta} \in S_{\hat{a}_{r+1, k}}} c_{\hat{\nu}} c_{\hat{\eta}}\left(\sum_{\zeta \in \varphi_{p_{i}+\cdots+p_{k}+1}^{-1}(\hat{\nu} \cup \hat{\eta})} \chi_{\zeta}\right)\right)
$$

where $c=(-1)^{\left|\hat{J}_{i} \cup \cdots \cup \hat{J}_{r}\right|\left(p_{r+1}+\cdots+p_{k}+1\right)}$ comes from the product of $\hat{a}_{i, r}$ and $\hat{a}_{r, k}$, as in Lemma 2.2, and $(-1)^{1+\overline{\operatorname{deg}}\left(\hat{a}_{i, r}\right)}=(-1)^{\left(p_{i}+\cdots+p_{r}\right)+\left|\hat{J}_{i} \cup \cdots \cup \hat{J}_{r}\right|}$.

Any simplex $\zeta \in \varphi_{p_{i}+\cdots+p_{k}+1}^{-1}(\hat{\nu} \cup \hat{\eta})$ is on $p_{i}+\cdots+p_{k}+2$ vertices and so can be written as $\nu \cup \eta$ for $\nu$ the restriction of $\zeta$ to its first $p_{i}+\cdots+p_{r}+1$ vertices, and $\eta$ the restriction of $\zeta$ to its last $p_{r+1}+\cdots+p_{k}+1$ vertices. Then $\nu \in \varphi_{p_{i}+\cdots+p_{r}}^{-1}(\hat{\nu})$ and $\eta \in \varphi_{p_{r+1}+\cdots+p_{k}}^{-1}(\hat{\eta})$. Furthermore, $\hat{\theta}_{i, k}(-1)^{1+\overline{\operatorname{deg}}\left(\hat{a}_{i, r}\right)} c=$ $(-1)^{\left(p_{i}+\cdots+p_{r}\right)} \hat{\theta}_{i, r} \hat{\theta}_{r+1, k}$. So (4.10) may be rewritten as

$$
\begin{aligned}
& d\left(a_{i, k}\right)=\sum_{r=i}^{k-1}(-1)^{\left(p_{i}+\cdots+p_{r}\right)} \theta_{i, k} \hat{\theta}_{i, r} \hat{\theta}_{r+1, k} . \\
& \cdot\left(\sum_{\hat{\nu} \in S_{\hat{a}_{i, r}}} \sum_{\hat{\eta} \in S_{\hat{a}_{r+1, k}}} c_{\hat{\nu}} c_{\hat{\eta}}\left(\sum_{\nu \in \varphi_{p_{i}+\cdots+p_{r}}^{-1}(\hat{\nu})} \sum_{\eta \in \varphi_{p_{r+1}+\cdots+p_{k}}^{-1}(\hat{\eta})} \chi_{\nu \cup \eta}\right)\right)
\end{aligned}
$$

Comparatively, the product $\sum_{r=i}^{k-1}(-1)^{1+\overline{\operatorname{deg}}\left(a_{i, r}\right)} a_{i, r} a_{r, k}$ is

$$
\begin{aligned}
& \sum_{r=i}^{k-1}(-1)^{1+\overline{\operatorname{deg}}\left(a_{i, r}\right)}(-1)^{\left|J_{i} \cup \cdots \cup J_{r}\right|\left(p_{r+1}+\cdots+p_{k}+1\right)} \theta_{i, r} \theta_{r+1, k} \hat{\theta}_{i, r} \hat{\theta}_{r+1, k} . \\
& \cdot\left(\sum_{\hat{\nu} \in S_{\hat{a}_{i, r}}} \sum_{\hat{\eta} \in S_{\hat{a}_{r+1, k}}} c_{\hat{\nu}} c_{\hat{\eta}}\left(\sum_{\nu \in \varphi_{p_{i}+\cdots+p_{r}}^{-1}(\hat{\nu})} \sum_{\eta \in \varphi_{p_{r+1}+\cdots+p_{k}}^{-1}(\hat{\eta})} \chi_{\nu \cup \eta}\right)\right)
\end{aligned}
$$

where the sign $(-1)^{\left|J_{i} \cup \cdots \cup J_{r}\right|\left(p_{r+1}+\cdots+p_{k}+1\right)}$ comes from the product of $a_{i, r}$ and $a_{r+1, k}$ as in Lemma 2.2, and $(-1)^{1+\overline{\operatorname{deg}}\left(a_{i, r}\right)}=(-1)^{\left(p_{i}+\cdots+p_{r}\right)+\left|J_{i} \cup \cdots \cup J_{r}\right|}$. Using 
the expression for $\theta_{i, k}$ in (4.6),

$$
(-1)^{1+\overline{\operatorname{deg}}\left(a_{i, r}\right)}(-1)^{\left|J_{i} \cup \cdots \cup J_{r}\right|\left(p_{r+1}+\cdots+p_{k}+1\right)} \theta_{i, r} \theta_{r+1, k}=(-1)^{\left(p_{i}+\cdots+p_{r}\right)} \theta_{i, k} .
$$

Therefore the expressions in (4.11) and (4.12) are equal.

Hence $d\left(a_{i, k}\right)=\sum_{r=i}^{k-1} \overline{\overline{a_{i, r}}} a_{r, k}$, and so $\left(a_{i, k}\right)$ is a defining system for the Massey product $\left\langle\alpha_{1}, \ldots, \alpha_{n}\right\rangle$.

Example 4.9. Let $J_{1}=\{1,2,3\}, \hat{J}_{1}=\{\hat{1}, \hat{2}\}, J_{2}=\{4,5\}$ and $\hat{J}_{2}=\{\hat{4}, \hat{5}\}$. Suppose that $\mathcal{K}_{J_{1} \cup J_{2}}$ and $\hat{\mathcal{K}}_{\hat{J}_{1} \cup \hat{J}_{2}}$ are the simplicial complexes shown below, where $\mathcal{K}_{J_{1} \cup J_{2}}$ maps onto $\hat{\mathcal{K}}_{\hat{J}_{1} \cup \hat{J}_{2}}$ by the edge contraction $\{2,3\} \mapsto\{\hat{2}\}$.

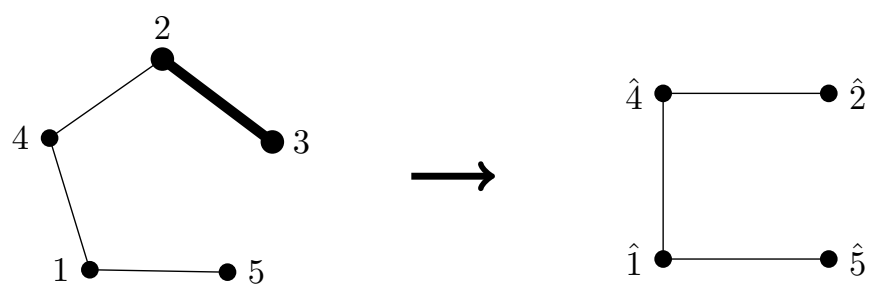

Suppose that $\hat{a}_{1}=\chi_{\hat{2}} \in C^{0}\left(\hat{\mathcal{K}}_{\hat{J}_{1}}\right), \hat{a}_{2}=\chi_{\hat{4}} \in C^{0}\left(\hat{\mathcal{K}}_{\hat{J}_{2}}\right)$, and $\hat{a}_{1,2}=-\chi_{\hat{2}} \in$ $C^{0}\left(\hat{\mathcal{K}}_{\hat{J}_{1} \cup \hat{J}_{2}}\right)$. Then $d\left(\hat{a}_{1,2}\right)=\chi_{\hat{2}, \hat{4}}=(-1)^{1+\overline{\operatorname{deg}} \hat{a}_{1}} \hat{a}_{1} \hat{a}_{2}$. By $(4.2), a_{1}=\chi_{2}+\chi_{3} \in$ $C^{0}\left(\mathcal{K}_{J_{1}}\right)$ and $a_{2}=\chi_{4} \in C^{0}\left(\mathcal{K}_{J_{2}}\right)$. By (4.5), $a_{1,2}=-\chi_{2}-\chi_{3} \in C^{0}\left(\mathcal{K}_{J_{1} \cup J_{2}}\right)$, since $\theta_{1,2}=1$. We check that $d\left(a_{1,2}\right)=\left(\chi_{2,4}+\chi_{2,3}\right)-\chi_{2,3}=\chi_{2,4}=(-1)^{1+\operatorname{deg}} a_{1} a_{1} a_{2}$. Hence $d\left(a_{1,2}\right)=\overline{\overline{a_{1}}} a_{2}$.

Example 4.10 (a). Let $\hat{\mathcal{K}}_{1}$ be a triangulation of $S^{1}$ on three vertices, $\{\hat{1}, \hat{2}, \hat{3}\}$. Let $\hat{\mathcal{K}}_{2}=\{\{\hat{5}\},\{\hat{6}\}\}$, and let $\hat{\mathcal{K}}_{3}=\{\{\hat{7}\},\{\hat{8}\}\}$. Let $\hat{\alpha}_{1}=\left[\chi_{\hat{1} \hat{3}}\right] \in \widetilde{H}^{1}\left(\hat{\mathcal{K}}_{1}\right), \hat{\alpha}_{2}=$ $\left[\chi_{\hat{5}}\right] \in \widetilde{H}^{0}\left(\hat{\mathcal{K}}_{2}\right)$ and $\hat{\alpha}_{3}=\left[\chi_{\hat{7}}\right] \in \widetilde{H}^{0}\left(\hat{\mathcal{K}}_{3}\right)$. Let $\hat{\mathcal{K}}=\operatorname{sd}_{\{\hat{5}, \hat{8}\}} \operatorname{sd}_{\{\hat{1}, \hat{3}, \hat{6}\}} \hat{\mathcal{K}}_{1} * \hat{\mathcal{K}}_{2} * \hat{\mathcal{K}}_{3}$ be a simplicial complex on the vertices $\{\hat{1}, \hat{2}, \hat{3}, \hat{5}, \hat{6}, \hat{7}, \hat{8}\}$. The simplicial complex $\hat{\mathcal{K}}_{\hat{1}, \hat{2}, \hat{3}, \hat{5}, \hat{6}}$ is shown in Figure 13b. By Theorem 3.17, there is a non-trivial triple Massey product $\left\langle\hat{\alpha}_{1}, \hat{\alpha}_{2}, \hat{\alpha}_{3}\right\rangle \subset H^{*}\left(\mathcal{Z}_{\hat{\mathcal{K}}}\right)$.

Let $\mathcal{K}$ be the simplicial complex on vertices $\{1, \ldots, 8\}$ that edge contracts to $\hat{\mathcal{K}}$ by contracting the edge $\{1,4\} \mapsto\{\hat{1}\}$, which satisfies the link condition. The contraction of the full subcomplex $\mathcal{K}_{J_{1} \cup J_{2}}$ is shown in Figure 13a. By Construction 4.6, there are cocycles $a_{1}=\chi_{13} \in C^{1}\left(\mathcal{K}_{J_{1}}\right), a_{2}=\chi_{5} \in C^{0}\left(\mathcal{K}_{J_{2}}\right)$, $a_{3}=\chi_{7} \in C^{0}\left(\mathcal{K}_{J_{3}}\right)$. The product $a_{1} a_{2}$ is $\chi_{13} \chi_{5}=(-1)^{4} \chi_{135}=\chi_{135}$. If $\hat{a}_{1,2}=$ $\chi_{\hat{1} \hat{3}}$, then using (4.5) we construct $a_{1,2}=\theta_{1,2} \hat{\theta}_{1,2} \chi_{13}=-\chi_{13}$. Alternatively, if $\hat{a}_{1,2}=-\chi_{\hat{1} \hat{b}}-\chi_{\hat{1} \hat{2}}-\chi_{\hat{1} \hat{5}}$, then $S_{\hat{a}_{1,2}}=\{\{\hat{1}, \hat{6}\},\{\hat{1}, \hat{2}\},\{\hat{1}, \hat{5}\}\}$. So $\varphi_{1}^{-1}(\{\hat{1}, \hat{b}\})=$ $\{\{1,6\},\{4,6\}\}, \varphi_{1}^{-1}(\{\hat{1}, \hat{2}\})=\{\{2,4\}\}$, and $\varphi_{1}^{-1}(\{\hat{1}, \hat{5}\})=\{\{1,5\},\{4,5\}\}$. By (4.5), $a_{1,2}=-\theta_{1,2} \hat{\theta}_{1,2}\left(\chi_{16}+\chi_{46}+\chi_{24}+\chi_{45}+\chi_{15}\right)=\chi_{16}+\chi_{46}+\chi_{24}+\chi_{45}+\chi_{15}$.

(b). In the proof of Proposition 4.8, we showed that the pullback of a defining system $\left\langle\hat{\alpha}_{1}, \ldots, \hat{\alpha}_{n}\right\rangle$ is a defining system for $\left\langle\alpha_{1}, \ldots, \alpha_{n}\right\rangle$. However there are defining systems for $\left\langle\alpha_{1}, \ldots, \alpha_{n}\right\rangle$ that are not pullbacks of defining systems 


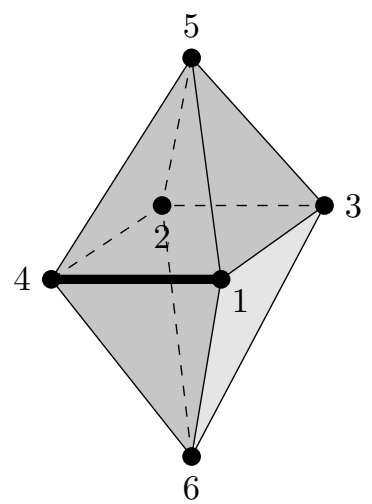

(a) The simplicial complex $\mathcal{K}_{1,2,3,4,5,6}$ which is missing the simplex $\{1,3,6\}$

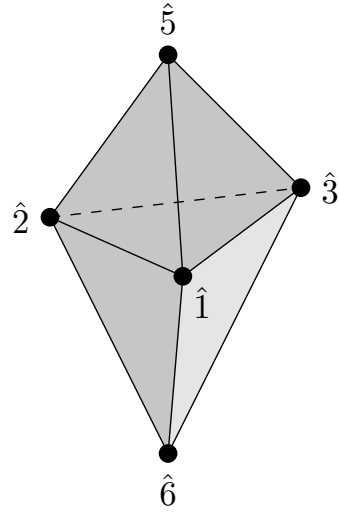

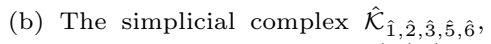
which is missing the simplex $\{\hat{1}, \hat{3}, \hat{6}\}$.

Figure 13: The simplicial complex $\mathcal{K}_{1,2,3,4,5,6}$ maps to $\hat{\mathcal{K}}_{\hat{1}, \hat{2}, \hat{3}, \hat{5}, \hat{6}}$ by contracting the edge $\{1,4\} \mapsto\{\hat{1}\}$.

for $\left\langle\hat{\alpha}_{1}, \ldots, \hat{\alpha}_{n}\right\rangle$. For example, let $a_{1}, a_{2}, a_{3}, \hat{a}_{1}, \hat{a}_{2}, \hat{a}_{3}$ be as in Part (a). Let $a_{1,2}=-\chi_{16}-\chi_{14}-\chi_{15}$. For the edge $\{1,4\} \in \mathcal{K},\{1,4\} \notin \varphi_{1}^{-1}(\hat{e})$ for any edge $\hat{e} \in \hat{\mathcal{K}}$, so $a_{1,2}$ is not a pullback of any $\hat{a}_{1,2}$. However for $\chi_{1} \in C^{*}\left(\mathcal{K}_{1,2,3,4,5,6}\right)$,

$$
\begin{aligned}
a_{1,2}-d\left(\chi_{1}\right) & =-\chi_{16}-\chi_{14}-\chi_{15}-\left(\chi_{16}+\chi_{14}+\chi_{15}+\chi_{13}\right) \\
& =-\chi_{13}=\theta_{1,2} \hat{\theta}_{1,2} \sum_{\tau \in \varphi_{p_{1}+p_{2}}^{-1}(\hat{1} \hat{3})} \chi_{\tau} .
\end{aligned}
$$

Therefore $a_{1,2}$ differs from the pullback of $\hat{a}_{1,2}=\chi_{\hat{1} \hat{3}}$ by a coboundary.

In order to prove that $\left\langle\alpha_{1}, \ldots, \alpha_{n}\right\rangle$ is non-trivial, we show that for every defining system for $\left\langle\alpha_{1}, \ldots, \alpha_{n}\right\rangle$, its associated cocycle is homologous to the pullback of an associated cocycle for a defining system for the non-trivial Massey product $\left\langle\hat{\alpha}_{1}, \ldots, \hat{\alpha}_{n}\right\rangle$.

Proposition 4.11. The $n$-Massey product $\left\langle\alpha_{1}, \ldots, \alpha_{n}\right\rangle$ is non-trivial.

Proof. Suppose that $\varphi: \mathcal{K} \rightarrow \hat{\mathcal{K}}$ is the contraction of just one edge $\{u, v\} \in \mathcal{K}$. By Construction 4.6, $\{u, v\} \subset J_{i}$ for $i \in\{1, \ldots, n\}$.

For $a_{i, i}=a_{i}$ the representative cocycle for $\alpha_{i}$ as defined in (4.2), let $\left(a_{i, k}\right)$ be a defining system for $\left\langle\alpha_{1}, \ldots, \alpha_{n}\right\rangle$,

$$
a_{i, k}=\sum_{\sigma \in S_{a_{i, k}}} c_{\sigma} \chi_{\sigma} \in C^{p_{i}+\cdots+p_{k}}\left(\mathcal{K}_{J_{i} \cup \cdots \cup J_{k}}\right) \text {. }
$$

We show that any defining system $\left(a_{i, k}\right)$ corresponds to a defining system $\left(\hat{a}_{i, k}\right)$ for $\left\langle\hat{\alpha}_{1}, \ldots, \hat{\alpha}_{n}\right\rangle$ in $H^{*}\left(\mathcal{Z}_{\hat{\mathcal{K}}}\right)$. There are two main stages to this proof. Firstly, for 
a defining system $\left(a_{i, k}\right)$ such that for any $\{i, k\},\{u, v\} \notin \sigma$ for any $\sigma \in S_{a_{i, k}}$, we construct a corresponding defining system $\left(\varphi^{*}\left(a_{i, k}\right)\right)$ for $\left\langle\hat{\alpha}_{1}, \ldots, \hat{\alpha}_{n}\right\rangle$. Secondly, for any other defining system $\left(a_{i, k}\right)$, we change $a_{i, k}$ to create a different defining system $\left(\widetilde{a}_{i, k}\right)$ for $\left\langle\alpha_{1}, \ldots, \alpha_{n}\right\rangle$ such that the associated cocycles are homologous and for any $\{i, k\},\{u, v\} \notin \sigma$ for any $\sigma \in S_{\widetilde{a}_{i, k}}$. Applying the first step to $\left(\widetilde{a}_{i, k}\right)$, we have a defining system $\left(\varphi^{*}\left(\widetilde{a}_{i, k}\right)\right)$ that corresponds to $\left(a_{i, k}\right)$.

For this first step, suppose that for any $\{i, k\},\{u, v\} \notin \sigma$ for any $\sigma \in$ $S_{a_{i, k}}$. We define a tool $\varphi^{*}$, which will only be well-defined for certain specified cochains such as $a_{i, k} \in C^{p}\left(\mathcal{K}_{J_{i} \cup \cdots \cup J_{k}}\right)$ or $a_{i, r} a_{r+1, k} \in C^{p}\left(\mathcal{K}_{J_{i} \cup \cdots \cup J_{k}}\right)$ where $p=p_{i}+\cdots+p_{k}$ or $p=p_{i}+\cdots+p_{k}+1$ respectively. We check three properties of $\varphi^{*}$ in order to construct a defining system $\left(\varphi^{*}\left(a_{i, k}\right)\right)$ for $\left\langle\hat{\alpha}_{1}, \ldots, \hat{\alpha}_{n}\right\rangle$. Let $a \in C^{p}\left(\mathcal{K}_{J_{i} \cup \cdots \cup J_{k}}\right)$ be a general cochain such that $\{u, v\} \notin \sigma$ for any $\sigma \in S_{a}$, where either $p=p_{i}+\cdots+p_{k}$ or $p=p_{i}+\cdots+p_{k}+1$. For $J \subset[m]$, let $\hat{J}=\varphi(J)$. Define

$$
\varphi^{*}(a)=c_{i, k} \sum_{\hat{\sigma} \in \varphi\left(S_{a}\right)} c_{\hat{\sigma}} \chi_{\hat{\sigma}} \in C^{p}\left(\hat{\mathcal{K}}_{\hat{J}_{i} \cup \cdots \cup \hat{J}_{k}}\right)
$$

where $c_{\hat{\sigma}}=c_{\sigma}$ for any $\sigma \in S_{a}$ such that $\varphi(\sigma)=\hat{\sigma}, c_{i, i}=1$ and

$c_{i, k}=(-1)^{\left(\left|J_{i}\right|-\left|\hat{J}_{i}\right|\right) p_{i+1}+\left(\left|J_{i} \cup J_{i+1}\right|-\left|\hat{J}_{i} \cup \hat{J}_{i+1}\right|\right) p_{i+2}+\cdots+\left(\left|J_{i} \cup \cdots \cup J_{k-1}\right|-\left|\hat{J}_{i} \cup \cdots \cup \hat{J}_{k-1}\right|\right) p_{k}}$.

(i) First note that for any constant $c^{\prime} \in \mathbf{k}$ and for $\chi_{\sigma}, \chi_{\tau}$ in $C^{p}\left(\mathcal{K}_{J_{i} \cup \ldots \cup J_{k}}\right)$ where $p$ is either $p_{i}+\cdots+p_{k}$ or $p_{i}+\cdots+p_{k}+1$ and $\{u, v\} \notin \sigma, \tau$,

$$
\begin{gathered}
\varphi^{*}\left(c^{\prime} c_{\sigma} \chi_{\sigma}\right)=c_{i, k} c^{\prime} c_{\sigma} \chi_{\varphi(\sigma)}=c^{\prime} \varphi^{*}\left(c_{\sigma} \chi_{\sigma}\right) \text { and } \\
\varphi^{*}\left(c_{\sigma} \chi_{\sigma}+c_{\tau} \chi_{\tau}\right)=c_{i, k}\left(c_{\sigma} \chi_{\varphi(\sigma)}+c_{\tau} \chi_{\varphi(\tau)}\right)=\varphi^{*}\left(c_{\sigma} \chi_{\sigma}\right)+\varphi^{*}\left(c_{\tau} \chi_{\tau}\right) .
\end{gathered}
$$

(ii) Next we show that $\varphi^{*}\left(d\left(a_{i, k}\right)\right)=d\left(\varphi^{*}\left(a_{i, k}\right)\right)$. Suppose that for a simplex $\sigma \in S_{a_{i, k}}$, there is a simplex $j \cup \sigma \in \mathcal{K}_{J_{i} \cup \cdots \cup J_{k}}$ for $j \in J_{i} \cup \cdots \cup J_{k} \backslash \sigma$ that is contracted. That is, $\{u, v\} \in j \cup \sigma$. By the definition of a defining system, $d\left(a_{i, k}\right)=\sum_{r=i}^{k-1} \overline{\overline{a_{i, r}}} a_{r, k}$. Therefore either $c_{\sigma} \varepsilon(j, j \cup \sigma) \chi_{j \cup \sigma}$ is cancelled by other terms in $d\left(a_{i, k}\right)$, or there exists $i \leqslant r<k$ and simplices $\tau \in S_{a_{i, r}}$, $\eta \in S_{a_{r+1, k}}$ such that $\tau \cup \eta=j \cup \sigma$. In the latter case, if $\{u, v\} \in j \cup \sigma$, then $\{u, v\} \in \tau \cup \eta$. This implies that either $\{u, v\} \in \tau$ or $\{u, v\} \in \eta$, since by construction $\{u, v\} \subset J_{i}$ for an $1 \leqslant i \leqslant n$ and $\tau \in S_{a_{i, r}} \subset J_{i} \cup \cdots \cup J_{r}$, $\eta \in S_{a_{r+1, k}} \subset J_{r+1} \cup \cdots \cup J_{k}$. This then contradicts the assumption that $\{u, v\} \notin \sigma$ for any $\sigma \in S_{a_{i, k}}$ and any $\{i, k\}$. Hence a summand of the form $c_{\sigma} \varepsilon(j, j \cup \sigma) \chi_{j \cup \sigma}$, where $\{u, v\} \in j \cup \sigma$, is cancelled out by other summands.

Let $a=\sum_{\sigma \in S_{a}} c_{\sigma} \chi_{\sigma} \in C^{p_{i}+\cdots+p_{k}}\left(\mathcal{K}_{J_{i} \cup \cdots \cup J_{k}}\right)$ be a cochain such that for any simplex $j \cup \sigma \in \mathcal{K}_{J_{i} \cup \cdots \cup J_{k}}$ for $\sigma \in S_{a}$ and $j \in J_{i} \cup \cdots \cup J_{k} \backslash \sigma$, either $c_{\sigma} \varepsilon(j, j \cup \sigma) \chi_{j \cup \sigma}$ is cancelled by other terms in $d(a)$ or $j \cup \sigma$ does not contract. Applying $\varphi^{*}$ to

$$
d(a)=\sum_{\sigma \in S_{a}} \sum_{\substack{j \in J_{i} \cup \ldots \cup J_{k} \backslash \sigma, j \cup \sigma \in \mathcal{K}_{J_{i} \cup \cdots \cup J_{k}}}} c_{\sigma} \varepsilon(j, j \cup \sigma) \chi_{j \cup \sigma},
$$


we write

$$
\varphi^{*}(d(a))=c_{i, k} \sum_{\hat{\sigma} \in \varphi\left(S_{a}\right)} c_{\hat{\sigma}}\left(\sum_{\substack{\hat{j} \in \hat{J}_{i} \cup \ldots \cup \hat{J}_{k} \backslash \hat{\sigma}, \hat{j} \cup \hat{\sigma} \in \hat{\mathcal{K}}_{\hat{J}_{i} \cup \cdots \cup \hat{J}_{k}}}} \varepsilon(\hat{j}, \hat{j} \cup \hat{\sigma}) \chi_{\hat{j} \cup \hat{\sigma}}\right)
$$

where $\varepsilon(j, j \cup \sigma)=\varepsilon(\hat{j}, \hat{j} \cup \hat{\sigma})$ due to the order on vertices in $\mathcal{K}$ and since $j \cup \sigma$ does not contract. Let $\hat{S}=\left\{\varphi(\sigma)\left|\sigma \in S_{a},\right| \varphi(\sigma) \mid=p_{i}+\cdots+p_{k}+1\right\}$ and let $b=\sum_{\hat{\sigma} \in \hat{S}} c_{\hat{\sigma}} \chi_{\hat{\sigma}} \in C^{p_{i}+\cdots+p_{k}}\left(\hat{\mathcal{K}}_{\hat{J}_{i} \cup \cdots \cup \hat{J}_{k}}\right)$. Then

$$
\varphi^{*}(d(a))=d(b)
$$

In particular, $\varphi^{*}\left(d\left(a_{i, k}\right)\right)=d\left(\varphi^{*}\left(a_{i, k}\right)\right)$.

(iii) We also show that

$$
\sum_{r=i}^{k-1} \overline{\overline{\varphi^{*}\left(a_{i, r}\right)}} \varphi^{*}\left(a_{r+1, k}\right)=\varphi^{*}\left(\sum_{r=i}^{k-1} \overline{\overline{a_{i, r}}} a_{r+1, k}\right) .
$$

Let $a_{i, r} \in C^{p_{i}+\cdots+p_{r}}\left(\mathcal{K}_{J_{i} \cup \cdots \cup J_{r}}\right)$ and $a_{r+1, k} \in C^{p_{r+1}+\cdots+p_{k}}\left(\mathcal{K}_{J_{r+1} \cup \cdots \cup J_{k}}\right)$ be represented by $\sum_{\tau \in S_{a_{i, r}}} c_{\tau} \chi_{\tau}$ and $\sum_{\eta \in S_{a_{r+1, k}}} c_{\eta} \chi_{\eta}$ respectively. The left hand side of $(4.16)$ is

$$
\begin{aligned}
& \sum_{r=i}^{k-1} \overline{\overline{\varphi^{*}\left(a_{i, r}\right)}} \varphi^{*}\left(a_{r+1, k}\right) \\
& \quad=\sum_{r=i}^{k-1}(-1)^{1+\overline{\operatorname{deg}} \varphi^{*}\left(a_{i, r}\right)}\left(c_{i, r} \sum_{\hat{\tau} \in \varphi\left(S_{a_{i, r}}\right)} c_{\tau} \chi_{\hat{\tau}}\right) \cdot\left(c_{r+1, k} \sum_{\hat{\eta} \in \varphi\left(S_{a_{r+1, k}}\right)} c_{\eta} \chi_{\hat{\eta}}\right) \\
& \quad=\sum_{r=i}^{k-1} C\left(\sum_{\hat{\tau} \in \varphi\left(S_{a_{i, r}}\right)} c_{\hat{\eta} \in \varphi\left(S_{a_{r+1, k}} c_{\eta}\right.} \chi_{\hat{\tau} \cup \hat{\eta}}\right)
\end{aligned}
$$

where

$$
C=(-1)^{1+\overline{\operatorname{deg}} \varphi^{*}\left(a_{i, r}\right)+\left|\hat{J}_{i} \cup \cdots \cup \hat{J}_{r}\right|\left(p_{r+1}+\cdots+p_{k}+1\right)} c_{i, r} c_{r+1, k} .
$$

Using the expressions for $c_{i, r}$ and $c_{r+1, k}$, and using $\overline{\operatorname{deg}} \varphi^{*}\left(a_{i, r}\right)=1+p_{i}+\cdots+$ $p_{r}+\left|\hat{J}_{i} \cup \cdots \cup \hat{J}_{r}\right|$,

$$
\begin{aligned}
& C=(-1)^{p_{i}+\cdots+p_{r}+\left|\hat{J}_{i} \cup \cdots \cup \hat{J}_{r}\right|+\left|\hat{J}_{i} \cup \cdots \cup \hat{J}_{r}\right|\left(p_{r+1}+\cdots+p_{k}+1\right)} \\
& \cdot(-1)^{\left(\left|J_{i}\right|-\left|\hat{J}_{i}\right|\right) p_{i+1}+\cdots+\left(\left|J_{i} \cup \cdots \cup J_{r-1}\right|-\left|\hat{J}_{i} \cup \cdots \cup \hat{J}_{r-1}\right|\right) p_{r}} \\
& \cdot(-1)^{\left(\left|J_{r+1}\right|-\left|\hat{J}_{r+1}\right|\right) p_{r+2}+\cdots+\left(\left|J_{r+1} \cup \cdots \cup J_{k-1}\right|-\left|\hat{J}_{r+1} \cup \cdots \cup \hat{J}_{k-1}\right|\right) p_{k}} \\
&=(-1)^{p_{i}+\cdots+p_{r}+\left(\left|J_{i}\right|-\left|\hat{J}_{i}\right|\right) p_{i+1}+\cdots+\left(\left|J_{i} \cup \cdots \cup J_{r-1}\right|-\left|\hat{J}_{i} \cup \cdots \cup \hat{J}_{r-1}\right|\right) p_{r}+\left|\hat{J}_{i} \cup \cdots \cup \hat{J}_{r}\right| p_{r+1}} \\
& \cdot(-1)^{\left(\left|J_{r+1}\right|-\left|\hat{J}_{i} \cup \cdots \cup \hat{J}_{r+1}\right|\right) p_{r+2}+\cdots+\left(\left|J_{r+1} \cup \cdots \cup J_{k-1}\right|-\left|\hat{J}_{i} \cup \cdots \cup \hat{J}_{k-1}\right|\right) p_{k}} \\
&=(-1)^{1+\overline{\operatorname{deg}} a_{i, r}}(-1)^{\left|J_{i} \cup \cdots \cup J_{r}\right|\left(p_{r+1}+\cdot+p_{k}+1\right)} c_{i, k} .
\end{aligned}
$$


By assumption, $\{u, v\} \notin \sigma$ for any $\sigma \in S_{a_{i, k}}$ and any $\{i, k\}$. Thus $\{u, v\} \notin \tau$ and $\{u, v\} \notin \eta$ for any $i \leqslant r<k$ and any simplices $\tau \in S_{a_{i, r}}, \eta \in S_{a_{r+1, k}}$. Also, $\{u, v\} \subset J_{i}$ for an index $1 \leqslant i \leqslant n$, so $\{u, v\} \notin \tau \cup \eta$. Hence $\varphi(\tau \cup \eta)=\varphi(\tau) \cup \varphi(\eta)$ is a $\left(p_{i}+\cdots+p_{k}+1\right)$-simplex. Therefore using the definition of $\varphi^{*}$, the property (i), and the fact that $\varphi(\tau \cup \eta)=\varphi(\tau) \cup \varphi(\eta)=\hat{\tau} \cup \hat{\eta}$,

$$
\begin{aligned}
& \sum_{r=i}^{k-1} \overline{\overline{\varphi^{*}\left(a_{i, r}\right)}} \varphi^{*}\left(a_{r+1, k}\right)= \\
& \quad=\sum_{r=i}^{k-1} C\left(\sum_{\hat{\tau} \in \varphi\left(S_{a_{i, r}}\right)} \sum_{\hat{\eta} \in \varphi\left(S_{a_{r+1, k}}\right)} c_{\tau} c_{\eta} \chi_{\hat{\tau} \cup \hat{\eta}}\right)=\varphi^{*}\left(\sum_{r=i}^{k-1} \overline{\overline{a_{i, r}}} a_{r+1, k}\right) .
\end{aligned}
$$

Using properties (i), (ii) and (iii), we prove that a defining system $\left(a_{i, k}\right)$ for $\left\langle\alpha_{1}, \ldots, \alpha_{n}\right\rangle$ and its associated cocycle $\omega$ are mapped by $\varphi^{*}$ onto a defining system for $\left\langle\hat{\alpha}_{1}, \ldots, \hat{\alpha}_{n}\right\rangle$ and its associated cocycle is $\varphi^{*}(\omega)$. By the definition of $a_{i}=a_{i, i}$ in (4.2), $\varphi^{*}\left(a_{i, i}\right)=\hat{a}_{i, i}=\hat{a}_{i}$. By properties (ii) and (iii), we see that

$$
d\left(\varphi^{*}\left(a_{i, k}\right)\right)=\varphi^{*}\left(d\left(a_{i, k}\right)\right)=\varphi^{*}\left(\sum_{r=i}^{k-1} \overline{\overline{a_{i, r}}} a_{r+1, k}\right)=\sum_{r=i}^{k-1} \overline{\overline{\varphi^{*}\left(a_{i, r}\right)}} \varphi^{*}\left(a_{r+1, k}\right) .
$$

Hence $\left(\varphi^{*}\left(a_{i, k}\right)\right)$ is a defining system for $\left\langle\hat{\alpha}_{1}, \ldots, \hat{\alpha}_{n}\right\rangle$ if $\left(a_{i, k}\right)$ is a defining system such that $\{u, v\} \notin \sigma$ for any $\sigma \in S_{a_{i, k}}$ and any pair $\{i, k\}$. Also, for the associated cocycle $\omega$ for $\left(a_{i, k}\right)$,

$$
\varphi^{*}(\omega)=\varphi^{*}\left(\sum_{r=1}^{n-1} \overline{\overline{a_{1, r}}} a_{r+1, n}\right)=\sum_{r=1}^{n-1} \overline{\overline{\varphi^{*}\left(a_{1, r}\right)}} \varphi^{*}\left(a_{r+1, n}\right)
$$

so $\varphi^{*}(\omega)$ is the associated cocycle for $\left(\varphi^{*}\left(a_{i, k}\right)\right)$.

Lastly we prove that $[\omega] \neq 0$. If $[\omega]=0$, then there is a cochain $a \in$ $C^{p_{1}+\cdots+p_{n}}\left(\mathcal{K}_{J_{1} \cup \cdots \cup J_{n}}\right)$ such that $\omega=d(a)$. Since $\{u, v\} \in J_{j}$ for some $j \in$ $\{1, \ldots, n\}$ and $\{u, v\} \notin \sigma$ for any $\sigma \in S_{a_{i, k}}$ and any $\{i, k\}$, no simplices in $S_{\omega}$ contract. Thus no simplices in $S_{d(a)}$. So by applying $\varphi^{*}$ and (4.15) from property (ii), $\varphi^{*}(\omega)=\varphi^{*}(d(a))=d(b)$ for a cochain $b \in C^{p_{i}+\cdots+p_{k}}\left(\hat{\mathcal{K}}_{\hat{J}_{i} \cup \cdots \cup \hat{J}_{k}}\right)$. So $\left[\varphi^{*}(\omega)\right]=0$, which contradicts the non-triviality of $\left\langle\hat{\alpha}_{1}, \ldots, \hat{\alpha}_{n}\right\rangle$. Therefore $[\omega] \neq 0$.

For the second stage of this proof, suppose that $\left(a_{i, k}\right)$ is a defining system for $\left\langle\alpha_{1}, \ldots, \alpha_{n}\right\rangle$ such that there is a pair of indices $\{i, k\}$ with $\{u, v\} \in \sigma$ for some $\sigma \in S_{a_{i, k}}$. We will define a new defining system $\left(\widetilde{a}_{i, k}\right)$ such that $\{u, v\} \notin \sigma$ for any $\sigma \in S_{\widetilde{a}_{i, k}}$ and such that $[\omega]=[\widetilde{\omega}]$ where $\omega$ and $\widetilde{\omega}$ are the associated cocycles for $\left(a_{i, k}\right)$ and $\left(\widetilde{a}_{i, k}\right)$, respectively.

The cocycle $a_{i}=a_{i, i}$ as defined in (4.2) is such that $\{u, v\} \notin \sigma$ for every $\sigma \in S_{a_{i}}$. Therefore, let $\{i, k\}$ be a pair of indices such that there is a simplex $\sigma \in S_{a_{i, k}}$ with $\{u, v\} \in \sigma$, and for every $i<i^{\prime \prime}<k^{\prime \prime}<k,\{u, v\} \notin \tau$ for any $\tau \in S_{a_{i^{\prime \prime}, k^{\prime \prime}}}$. Let $\sigma \in S_{a_{i, k}}$ be a simplex such that $\{u, v\} \in \sigma$, and let $c_{\sigma}$ be 
the non-zero coefficient of $\chi_{\sigma}$ in $a_{i, k}$. Then for every pair $\left\{i^{\prime}, k^{\prime}\right\} \subset[n]$, let $c=(-1)^{\overline{\operatorname{deg}} a_{i, k}} c_{\sigma} \varepsilon(u, \sigma)$ and define

$$
\widetilde{a}_{i^{\prime}, k^{\prime}}= \begin{cases}a_{i, k}-c_{\sigma} \varepsilon(u, \sigma) d\left(\chi_{\sigma \backslash u}\right) & \text { if } i^{\prime}=i<k=k^{\prime}, \\ a_{i^{\prime}, k}+c_{\sigma} \varepsilon(u, \sigma) a_{i^{\prime}, i-1} \chi_{\sigma \backslash u} & \text { if } i^{\prime}<i<k=k^{\prime}, \\ a_{i, k^{\prime}}+c \chi_{\sigma \backslash u} a_{k+1, k^{\prime}} & \text { if } i^{\prime}=i<k<k^{\prime}, \\ a_{i^{\prime}, k^{\prime}} & \text { if } i^{\prime}<i<k<k^{\prime} \text { or } i<i^{\prime}<k^{\prime}<k\end{cases}
$$

where $\chi_{\sigma \backslash u} \in C^{p_{i}+\cdots p_{k}-1}\left(\mathcal{K}_{J_{i} \cup \cdots \cup J_{k}}\right)$. We show that $\left(\widetilde{a}_{i^{\prime}, k^{\prime}}\right)$ is a defining system for $\left\langle\alpha_{1}, \ldots, \alpha_{n}\right\rangle$. Firstly since $k-i>1, \widetilde{a}_{i^{\prime}, i^{\prime}}=a_{i^{\prime}, i^{\prime}}$ for every $i^{\prime} \in[n]$. We also need to show that $d\left(\widetilde{a}_{i^{\prime}, k^{\prime}}\right)=\sum_{r=i^{\prime}}^{k^{\prime}-1} \overline{\overline{\widetilde{a}_{i^{\prime}, r}}} \widetilde{a}_{r+1, k^{\prime}}$ for every $\left\{i^{\prime}, k^{\prime}\right\}$.

(i) For $i<i^{\prime}<k^{\prime}<k$, we have $\widetilde{a}_{i^{\prime}, k^{\prime}}=a_{i^{\prime}, k^{\prime}}$ so

$$
d\left(\widetilde{a}_{i^{\prime}, k^{\prime}}\right)=d\left(a_{i^{\prime}, k^{\prime}}\right)=\sum_{r=i^{\prime}}^{k^{\prime}-1} \overline{\overline{a_{i^{\prime}, r}}} a_{r+1, k^{\prime}}=\sum_{r=i^{\prime}}^{k^{\prime}-1} \overline{\overline{\widetilde{a}_{i^{\prime}, r}}} \widetilde{a}_{r+1, k^{\prime}} .
$$

(ii) For $i^{\prime}=i<k=k^{\prime}$,

$$
d\left(\widetilde{a}_{i, k}\right)=d\left(a_{i, k}-c_{\sigma} \varepsilon(u, \sigma) d\left(\chi_{\sigma \backslash u}\right)\right)=d\left(a_{i, k}\right) .
$$

Also $d\left(\chi_{\sigma \backslash u}\right) \in C^{p_{i}+\cdots p_{k}}\left(\mathcal{K}_{J_{i} \cup \cdots \cup J_{k}}\right)$ since $\chi_{\sigma \backslash u} \in C^{p_{i}+\cdots p_{k}-1}\left(\mathcal{K}_{J_{i} \cup \cdots \cup J_{k}}\right)$. Hence $\tilde{a}_{i, k} \in C^{p_{i}+\cdots p_{k}}\left(\mathcal{K}_{J_{i} \cup \cdots \cup J_{k}}\right)$ and $\overline{\operatorname{deg}} \widetilde{a}_{i, k}=\overline{\operatorname{deg}} a_{i, k}$. Additionally,

$$
d\left(\chi_{\sigma \backslash u}\right)=\sum_{\substack{j \in J_{i} \cup \ldots \cup J_{k} \backslash(\sigma \backslash u), j \cup \sigma \backslash u \in \mathcal{K}_{J}}} \varepsilon(j, j \cup \sigma \backslash u) \chi_{j \cup \sigma \backslash u} .
$$

So $\chi_{\sigma}$ is the only summand of $d\left(\chi_{\sigma \backslash u}\right)$ such that $\{u, v\} \in \sigma$. Thus $a_{i, k}-$ $c_{\sigma} \varepsilon(u, \sigma) d\left(\chi_{\sigma \backslash u}\right)$ no longer contains the summand $\chi_{\sigma}$ and also

$$
\left|\left\{\tau \in S_{\widetilde{a}_{i, k}} \mid\{u, v\} \in \tau\right\}\right|<\left|\left\{\tau \in S_{a_{i, k}} \mid\{u, v\} \in \tau\right\}\right| .
$$

(iii) Next, for $i^{\prime}<i<k=k^{\prime}$, we have $a_{i^{\prime}, i-1} \in C^{p_{i^{\prime}}+\cdots+p_{i-1}}\left(\mathcal{K}_{J_{i^{\prime}} \cup \cdots \cup J_{i-1}}\right)$. So $a_{i^{\prime}, i-1} \chi_{\sigma \backslash u} \in C^{p_{i^{\prime}}+\cdots+p_{k}}\left(\mathcal{K}_{J_{i^{\prime}} \cup \cdots \cup J_{k}}\right)$. Hence $\widetilde{a}_{i^{\prime}, k} \in C^{p_{i^{\prime}}+\cdots+p_{k}}\left(\mathcal{K}_{J_{i^{\prime}} \cup \cdots \cup J_{k}}\right)$. 
Also,

$$
\begin{aligned}
d\left(\widetilde{a}_{i^{\prime}, k}\right)= & d\left(a_{i^{\prime}, k}+c_{\sigma} \varepsilon(u, \sigma) a_{i^{\prime}, i-1} \chi_{\sigma \backslash u}\right) \\
= & \sum_{r=i^{\prime}}^{k-1} \overline{\overline{a_{i^{\prime}, r}}} a_{r+1, k}+ \\
& \quad+c_{\sigma} \varepsilon(u, \sigma)\left(\sum_{r=i^{\prime}}^{i-2} \overline{\overline{a_{i^{\prime}, r}}} a_{r+1, i-1}\right) \chi_{\sigma \backslash u}-c_{\sigma} \varepsilon(u, \sigma) \overline{\overline{a_{i^{\prime}, i-1}}} d\left(\chi_{\sigma \backslash u}\right) \\
= & \sum_{r=i^{\prime}}^{i-2} \overline{\overline{a_{i^{\prime}, r}}}\left(a_{r+1, k}+c_{\sigma} \varepsilon(u, \sigma) a_{r+1, i-1} \chi_{\sigma \backslash u}\right)+ \\
\quad & \quad \overline{\overline{a_{i^{\prime}, i-1}}}\left(a_{i, k}-c_{\sigma} \varepsilon(u, \sigma) d\left(\chi_{\sigma \backslash u}\right)\right)+\sum_{r=i}^{k-1} \overline{\overline{a_{i^{\prime}, r}}} a_{r+1, k} \\
= & \sum_{r=i^{\prime}}^{k-1} \overline{\overline{\tilde{a}_{i^{\prime}, r}}} \widetilde{a}_{r+1, k} .
\end{aligned}
$$

(iv) For $i^{\prime}=i<k<k^{\prime}$, we have $\widetilde{a}_{i, k^{\prime}} \in C^{p_{i}+\cdots+p_{k^{\prime}}}\left(\mathcal{K}_{J_{i} \cup \cdots \cup J_{k^{\prime}}}\right)$ since $\chi_{\sigma \backslash u} a_{k+1, k^{\prime}} \in C^{p_{i}+\cdots+p_{k^{\prime}}}\left(\mathcal{K}_{J_{i} \cup \cdots \cup J_{k^{\prime}}}\right)$. Furthermore, $d\left(\widetilde{a}_{i, k^{\prime}}\right)$ is

$$
\begin{aligned}
& d\left(a_{i, k^{\prime}}+(-1)^{\overline{\operatorname{deg}} a_{i, k}} c_{\sigma} \varepsilon(u, \sigma) \chi_{\sigma \backslash u} a_{k+1, k^{\prime}}\right) \\
& =\sum_{r=i}^{k^{\prime}-1} \overline{\overline{a_{i, r}}} a_{r+1, k^{\prime}}+(-1)^{\overline{\operatorname{deg}} a_{i, k}} c_{\sigma} \varepsilon(u, \sigma) d\left(\chi_{\sigma \backslash u}\right) a_{k+1, k^{\prime}} .
\end{aligned}
$$

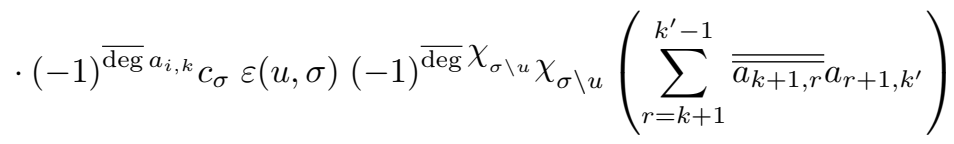

$$
\begin{aligned}
& =\sum_{r=i}^{k-1} \overline{\overline{a_{i, r}}} a_{r+1, k}-(-1)^{\overline{\operatorname{deg}} a_{i, k}}\left(a_{i, k}-c_{\sigma} \varepsilon(u, \sigma) d\left(\chi_{\sigma \backslash u}\right)\right) a_{k+1, k^{\prime}}+
\end{aligned}
$$

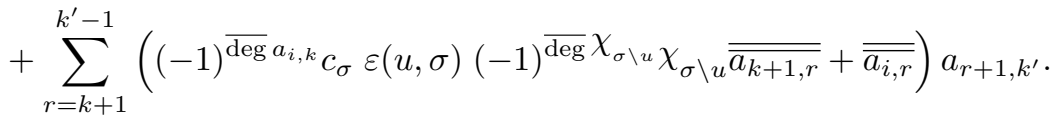

More specifically, let $c=(-1)^{\overline{\operatorname{deg}} a_{i, k}} c_{\sigma} \varepsilon(u, \sigma)$. Then in the last sum,

$$
\begin{aligned}
c(-1)^{\overline{\operatorname{deg}}} \chi_{\sigma \backslash u} \chi_{\sigma \backslash u} \overline{\overline{a_{k+1, r}}} & \\
& =(-1)^{p_{i}+\cdots+p_{k}+\left|J_{i} \cup \cdots \cup J_{k}\right|+p_{k+1}+\cdots+p_{r}+\left|J_{k+1} \cup \cdots \cup J_{r}\right|} c \chi_{\sigma \backslash u} a_{k+1, r} \\
& =(-1)^{1+\overline{\operatorname{deg}} a_{i, r}} c \chi_{\sigma \backslash u} a_{k+1, r} .
\end{aligned}
$$


Therefore

$$
\begin{aligned}
d\left(\widetilde{a}_{i, k^{\prime}}\right)= & \sum_{r=i}^{k-1} \overline{\overline{a_{i, r}}} a_{r+1, k}+(-1)^{1+\overline{\operatorname{deg}} a_{i, k}}\left(a_{i, k}-c_{\sigma} \varepsilon(u, \sigma) d\left(\chi_{\sigma \backslash u}\right)\right) a_{k+1, k^{\prime}} \\
& \quad+\sum_{r=k+1}^{k^{\prime}-1}(-1)^{1+\overline{\operatorname{deg}} a_{i, r}}\left(c \chi_{\sigma \backslash u} a_{k+1, r}+a_{i, r}\right) a_{r+1, k^{\prime}} \\
= & \sum_{r=i^{\prime}}^{k-1} \overline{\overline{\widetilde{a}}_{i^{\prime}, r}} \widetilde{a}_{r+1, k} .
\end{aligned}
$$

(v) Lastly when $i^{\prime}<i<k<k^{\prime}, \widetilde{a}_{i^{\prime}, k^{\prime}}=a_{i^{\prime}, k^{\prime}}$ and we want to show that $d\left(\widetilde{a}_{i^{\prime}, k^{\prime}}\right)=\sum_{r=i^{\prime}}^{k^{\prime}-1} \overline{\widetilde{\widetilde{a}}_{i^{\prime}, r}} \widetilde{a}_{r+1, k^{\prime}}$. The right hand side is

$$
\sum_{r=i^{\prime}}^{k^{\prime}-1} \overline{\overline{\bar{a}_{i^{\prime}, r}}} \widetilde{a}_{r+1, k^{\prime}}=\overline{\overline{a_{i^{\prime}, i-1}}} \widetilde{a}_{i, k^{\prime}}+\overline{\overline{\bar{a}_{i^{\prime}, k}}} a_{k+1, k^{\prime}}+\sum_{r \in\left\{i^{\prime}, \ldots, \widehat{i-1}, \ldots, \widehat{k}, \ldots k^{\prime}-1\right\}} \overline{\overline{a_{i^{\prime}, r}}} a_{r+1, k^{\prime}}
$$

where $\widehat{ }$ denotes omission. By expanding $\widetilde{a}_{i, k^{\prime}}, \widetilde{a}_{i^{\prime}, k}$ and the signs in this expression, $\sum_{r=i^{\prime}}^{k^{\prime}-1} \overline{\widetilde{a}_{i^{\prime}, r}} \widetilde{a}_{r+1, k^{\prime}}$ is

$$
\begin{aligned}
& (-1)^{1+\overline{\operatorname{deg}} a_{i^{\prime}, i-1}} a_{i^{\prime}, i-1}\left(a_{i, k^{\prime}}+(-1)^{\overline{\operatorname{deg}} a_{i, k}} c_{\sigma} \varepsilon(u, \sigma) \chi_{\sigma \backslash u} a_{k+1, k^{\prime}}\right)+ \\
& +(-1)^{1+\overline{\operatorname{deg}} a_{i^{\prime}, k}}\left(a_{i^{\prime}, k}+c_{\sigma} \varepsilon(u, \sigma) a_{i^{\prime}, i-1} \chi_{\sigma \backslash u}\right) a_{k+1, k^{\prime}}+ \\
& +\sum_{r \in\left\{i^{\prime}, \ldots, \widehat{i-1}, \ldots, \widehat{k}, \ldots k^{\prime}-1\right\}} \overline{\overline{a_{i^{\prime}, r}}} a_{r+1, k^{\prime}}
\end{aligned}
$$

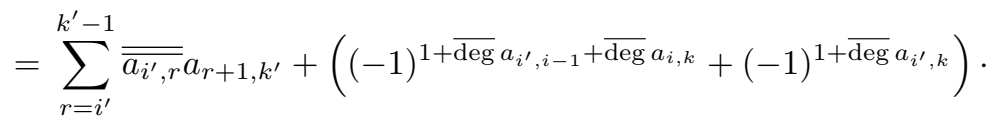

$$
\begin{aligned}
& \cdot c_{\sigma} \varepsilon(u, \sigma) a_{i^{\prime}, i-1} \chi_{\sigma \backslash u} a_{k+1, k^{\prime}} \\
& =d\left(a_{i^{\prime}, k^{\prime}}\right)+\left((-1)^{\overline{\operatorname{deg}} a_{i^{\prime}, k}}+(-1)^{1+\overline{\operatorname{deg}} a_{i^{\prime}, k}}\right) c_{\sigma} \varepsilon(u, \sigma) a_{i^{\prime}, i-1} \chi_{\sigma \backslash u} a_{k+1, k^{\prime}} \\
& =d\left(a_{i^{\prime}, k^{\prime}}\right)=d\left(\widetilde{a}_{i^{\prime}, k^{\prime}}\right)
\end{aligned}
$$

since $\overline{\operatorname{deg}} a_{i^{\prime}, k}=\left|J_{i^{\prime}} \cup \cdots \cup J_{k}\right|+p_{i^{\prime}}+\cdots p_{k}+1=\overline{\operatorname{deg}} \widetilde{a}_{i^{\prime}, k}$.

Therefore for all $\left\{i^{\prime}, k^{\prime}\right\}, \widetilde{a}_{i^{\prime}, k^{\prime}} \in C^{p_{i^{\prime}}+\cdots+p_{k^{\prime}}}\left(\mathcal{K}_{J_{i^{\prime}} \cup \cdots \cup J_{k^{\prime}}}\right)$ and $d\left(\widetilde{a}_{i^{\prime}, k^{\prime}}\right)=$ $\sum_{r=i^{\prime}}^{k^{\prime}-1} \overline{\widetilde{\widetilde{a}}_{i^{\prime}, r}} \widetilde{a}_{r+1, k^{\prime}}$. So $\left(\widetilde{a}_{i^{\prime}, k^{\prime}}\right)$ is a defining system for $\left\langle\alpha_{1}, \ldots, \alpha_{n}\right\rangle$. Also $\sigma \notin \tau$ for any $\tau \in S_{\widetilde{a}_{i^{\prime}, k^{\prime}}}$ and any $\left\{i^{\prime}, k^{\prime}\right\}$. The associated cocycle $\widetilde{\omega}$ for this defining system is given by $\sum_{r=1}^{n-1} \overline{\overline{\widetilde{a}_{1, r}}} \widetilde{a}_{r+1, n}$. By calculating $\sum_{r=1}^{n-1} \overline{\overline{\widetilde{a}_{1, r}}} \widetilde{a}_{r+1, n}$ in a similar manner as in the above calculations,

$$
\widetilde{\omega}= \begin{cases}\omega & \text { if } i \neq 1, k \neq n, \\ \omega+c_{\sigma} \varepsilon(u, \sigma) d\left(a_{i^{\prime}, i-1} \chi_{\sigma \backslash u}\right) & \text { if } 1=i<k=n, \\ \omega-(-1)^{1+\operatorname{deg} a_{i, k}} c_{\sigma} \varepsilon(u, \sigma) d\left(\chi_{\sigma \backslash u} a_{k+1, k^{\prime}}\right) & \text { if } 1=i<k<n\end{cases}
$$


where $\omega$ is the associated cocycle for $\left(a_{i^{\prime}, k^{\prime}}\right)$. So $[\widetilde{\omega}]=[\omega]$. Therefore $[\widetilde{\omega}]=0$ if and only if $[\omega]=0$.

If there is cochain $\widetilde{a}_{i^{\prime}, k^{\prime}}$ in the defining system $\left(\widetilde{a}_{i, k}\right)$ such that there is a a simplex $\sigma \in S_{\widetilde{a}_{i^{\prime}, k^{\prime}}}$ with $\{u, v\} \in \sigma$, then we repeat the above procedure to construct $\left(\widetilde{\widetilde{a}}_{i^{\prime}, k^{\prime}}\right)$, etc. After a finite number of iterations, we obtain a defining system $\left(\widetilde{a}_{i^{\prime}, k^{\prime}}\right)$ such that for any $\left\{i^{\prime}, k^{\prime}\right\}$ and any simplex $\sigma \in S_{\widetilde{a}_{i^{\prime}, k^{\prime}}}$, the edge $\{u, v\}$ is not contained in $\sigma$. Then we can construct a defining system $\left(\varphi^{*}\left(\widetilde{a}_{i^{\prime}, k^{\prime}}\right)\right)$ for $\left\langle\hat{\alpha}_{1}, \ldots, \hat{\alpha}_{n}\right\rangle$. Let $\omega$ and $\widetilde{\omega}$ be the associated cocycles for $\left(a_{i, k}\right)$ and $\left(\widetilde{a}_{i, k}\right)$, respectively. If $[\omega]=[\widetilde{\omega}]=0$, then $\left[\varphi^{*}(\widetilde{\omega})\right]=0$, which contradicts the assumption that $\left\langle\hat{\alpha}_{1}, \ldots, \hat{\alpha}_{n}\right\rangle$ is non-trivial. Hence if $\left\langle\hat{\alpha}_{1}, \ldots, \hat{\alpha}_{n}\right\rangle$ is non-trivial, then $\left\langle\alpha_{1}, \ldots, \alpha_{n}\right\rangle$ is non-trivial.

If $\mathcal{K} \rightarrow \hat{\mathcal{K}}$ by a series of more than one edge contractions, we repeat the steps in this proof for each edge contraction in turn.

Putting together Proposition 4.8 and Proposition 4.11, we have proved the following statement.

Theorem 4.12. Let $\hat{\mathcal{K}}$ be a simplicial complex with a non-trivial n-Massey product in $H^{*}\left(\mathcal{Z}_{\hat{\mathcal{K}}}\right)$. Let $\mathcal{K}$ be a simplicial complex that maps onto $\hat{\mathcal{K}}$ by a series of edge contractions $\varphi: \mathcal{K} \rightarrow \hat{\mathcal{K}}$ that satisfy the link condition. Then there is a non-trivial $n$-Massey product in $H^{*}\left(\mathcal{Z}_{\mathcal{K}}\right)$.

By construction, $\alpha_{i} \in H^{\left|J_{i}\right|+p_{i}+1}\left(\mathcal{Z}_{\mathcal{K}}\right)$ and $\hat{\alpha}_{i} \in H^{\left|\hat{J}_{i}\right|+p_{i}+1}\left(\mathcal{Z}_{\hat{\mathcal{K}}}\right)$ with $\left|J_{i}\right| \geqslant$ $\left|\hat{J}_{i}\right|$ for each $i$. Hence the degree of $\left\langle\alpha_{1}, \ldots, \alpha_{n}\right\rangle \subset H^{\left|J_{1} \cup \cdots \cup J_{n}\right|+\left(p_{1}+\cdots+p_{n}\right)+2}\left(\mathcal{Z}_{\mathcal{K}}\right)$ is greater than the degree of $\left\langle\hat{\alpha}_{1}, \ldots, \hat{\alpha}_{n}\right\rangle \subset H^{\left|\hat{J}_{1} \cup \cdots \cup \hat{J}_{n}\right|+\left(p_{1}+\cdots+p_{n}+1\right)+1}\left(\mathcal{Z}_{\hat{\mathcal{K}}}\right)$. Also, if $\left\langle\hat{\alpha}_{1}, \ldots, \hat{\alpha}_{n}\right\rangle$ has non-trivial indeterminacy, then $\left\langle\alpha_{1}, \ldots, \alpha_{n}\right\rangle$ also has non-trivial indeterminacy. As noted earlier, the converse does not necessarily hold: the pullback Massey product in $H^{*}\left(\mathcal{Z}_{\mathcal{K}}\right)$ might have non-trivial indeterminacy even if it is a pullback of a uniquely defined Massey product in $H^{*}\left(\mathcal{Z}_{\hat{\mathcal{K}}}\right)$.

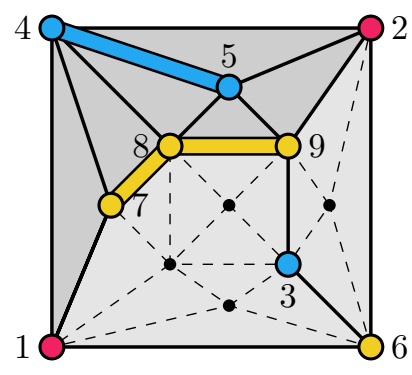

(a) A full subcomplex $\mathcal{K} \subset \mathcal{K}_{P}$, when $P$ is a truncated octahedron

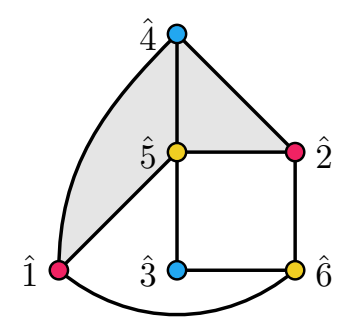

(b) A simplicial complex $\hat{\mathcal{K}}$ such that $\mathcal{Z}_{\hat{\mathcal{K}}}$ has non-trivial triple Massey product with indeterminacy.

Figure 14: Edge contraction example 
Example 4.13. Let $\hat{\mathcal{K}}$ be the simplicial complex in Figure 14b. Since the 1 -skeleton of $\hat{\mathcal{K}}$ is one of the obstruction graphs in the classification of lowest degree non-trivial triple Massey products [15], there is a non-trivial triple Massey product $\left\langle\hat{\alpha}_{1}, \hat{\alpha}_{2}, \hat{\alpha}_{3}\right\rangle \subset H^{8}\left(\mathcal{Z}_{\hat{\mathcal{K}}}\right)$ where $\hat{\alpha}_{1} \in \widetilde{H}^{0}\left(\hat{\mathcal{K}}_{\hat{1} \hat{2}}\right), \hat{\alpha}_{2} \in \widetilde{H}^{0}\left(\hat{\mathcal{K}}_{\hat{3} \hat{4}}\right)$ and $\hat{\alpha}_{3} \in \widetilde{H}^{0}\left(\hat{\mathcal{K}}_{\hat{5} \hat{b}}\right)$. This Massey product has non-trivial indeterminacy, since the indeterminacy of this triple Massey product is given by $\hat{\alpha}_{1} \cdot \widetilde{H}^{0}\left(\hat{\mathcal{K}}_{\hat{3} \hat{4} \hat{5} \hat{b}}\right)+\hat{\alpha}_{3}$. $\widetilde{H}^{0}\left(\hat{\mathcal{K}}_{\hat{1} \hat{2} \hat{3} \hat{4}}\right)=\hat{\alpha}_{3} \cdot \widetilde{H}^{0}\left(\hat{\mathcal{K}}_{\hat{1} \hat{2} \hat{3} \hat{4}}\right)$.

Let $\mathcal{K}$ be the simplicial complex on 9 vertices in Figure 14a. Let $\varphi: \mathcal{K} \rightarrow \hat{\mathcal{K}}$ be the simplicial map that takes $i \mapsto \hat{i}$ for $i=1,2,3,6$ and contracts the bold coloured edges $\{4,5\} \mapsto \hat{4},\{7,8\},\{8,9\} \mapsto \hat{5}$. By Theorem 4.12 and Construction 4.6, there is a non-trivial Massey product $\left\langle\alpha_{1}, \alpha_{2}, \alpha_{3}\right\rangle \subset H^{11}\left(\mathcal{Z}_{\mathcal{K}}\right)$ where $\alpha_{1} \in \widetilde{H}^{0}\left(\mathcal{K}_{12}\right), \alpha_{2} \in \widetilde{H}^{0}\left(\mathcal{K}_{345}\right)$ and $\alpha_{3} \in \widetilde{H}^{0}\left(\mathcal{K}_{6789}\right)$. Also the indeterminacy of this Massey product is non-trivial since it is given by $\alpha_{1} \cdot \widetilde{H}^{0}\left(\mathcal{K}_{3456789}\right)+\alpha_{3}$. $\widetilde{H}^{0}\left(\mathcal{K}_{12345}\right)=\alpha_{3} \cdot \widetilde{H}^{0}\left(\mathcal{K}_{12345}\right)$.

For any simple polytope $P$, define $\mathcal{K}_{P}=\partial\left(P^{*}\right)$ to be the boundary of the dual polytope. This is a simplicial complex and the moment-angle complex $\mathcal{Z}_{P}=\mathcal{Z}_{\mathcal{K}_{P}}$ is a moment-angle manifold. The simplicial complex $\mathcal{K}$ in Figure 14a is a full-subcomplex of $\mathcal{K}_{P}$ when $P$ is a truncated octahedron, otherwise known as the 3-dimensional permutahedron. A truncated octahedron is a 3-dimensional simple polytope whose facets are 6 squares and 8 hexagons, so there are 6 vertices of $\mathcal{K}_{P}$ with valency 4 and 8 with valency 6 . Since $\mathcal{K} \subset \mathcal{K}_{P}$, the non-trivial Massey product in $H^{*}\left(\mathcal{Z}_{\mathcal{K}}\right)$ lifts to a non-trivial Massey product in $H^{*}\left(\mathcal{Z}_{P}\right)$ with non-trivial indeterminacy. Hence we found a non-trivial Massey product in $H^{*}\left(\mathcal{Z}_{P}\right)$ using only Theorem 4.12 and the classification of lowest-degree nontrivial triple Massey products in $[12,15]$. This technique also recovers the first example of a triple Massey product in $H^{*}\left(\mathcal{Z}_{P}\right)$ that was given in [20, Lemma 4.9(2)], where the constructed full subcomplex edge contracts to one of the obstruction graphs that give trivial indeterminacy.

Example 4.14. A Pogorelov polytope is a 3-dimensional polytope that can be realised in hyperbolic (Lobachevsky) space as a bounded right-angled polytope. The Pogorelov class is large and includes all fullerenes, whose facets are pentagons and hexagons. Zhuravleva [26, Theorem 3.2] showed that for any Pogorelov polytope $P, \mathcal{K}_{P}=\partial\left(P^{*}\right)$ has a full subcomplex $\mathcal{K}$ as shown in Figure 15a. This full subcomplex was used to explicitly construct a nontrivial Massey product $\left\langle\alpha_{1}, \alpha_{2}, \alpha_{3}\right\rangle \subset H^{*}\left(\mathcal{Z}_{P}\right)$ where $\alpha_{1} \in \widetilde{H}^{0}\left(\mathcal{K}_{567}\right), \alpha_{2} \in$ $\widetilde{H}^{0}\left(\mathcal{K}_{2 b_{0} \ldots b_{n}}\right)$ and $\alpha_{3} \in \widetilde{H}^{0}\left(\mathcal{K}_{34}\right)$. moment-angle manifolds $\mathcal{Z}_{P}$ have a nontrivial triple Massey product using the full subcomplex in Figure 15a.

Edge contracting the coloured edges of $\mathcal{K},\left\{b_{i}, b_{i+1}\right\} \mapsto \hat{b}_{0},\{6,7\} \mapsto \hat{6}$, we obtain the simplicial complex in Figure 15b. This simplicial complex has a non-trivial triple Massey product, since its 1-skeleton is one of the obstruction graphs from the classification in $[12,15]$. Since the edge contractions satisfy the link condition, Theorem 4.12 gives an alternative proof of non-trivial triple Massey products in Zhuravleva's work. 


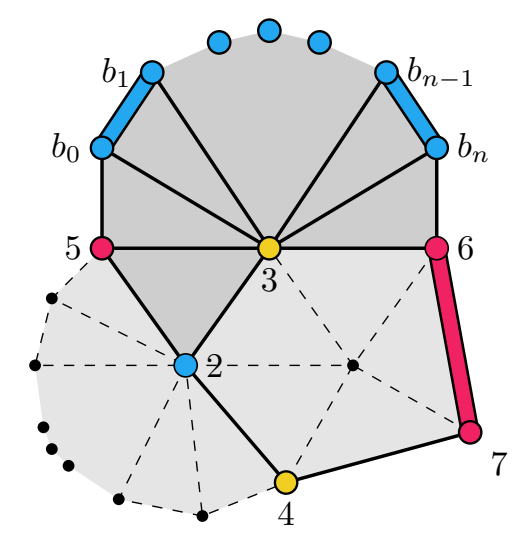

(a) A full subcomplex $\mathcal{K} \subset \mathcal{K}_{P}$ when $P$ is any Pogorelov polytope [26]

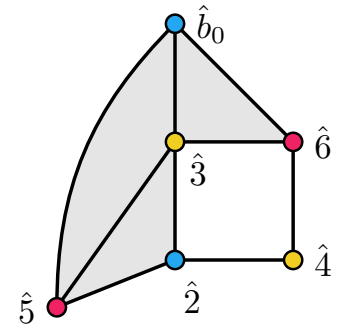

(b) An edge-contracted full subcomplex of a simplicial complex corresponding to any Pogorelov polytope

Figure 15: Massey products in Pogorelov polytopes

\subsection{Massey products constructed by edge stretching}

For an edge contraction $\mathcal{K} \mapsto \hat{\mathcal{K}}$ that satisfies the link condition, we call the inverse $\hat{\mathcal{K}} \mapsto \mathcal{K}$ edge stretching.

Corollary 4.15. Let $\hat{\mathcal{K}}$ be a simplicial complex with a non-trivial n-Massey product $\left\langle\hat{\alpha}_{1}, \ldots, \hat{\alpha}_{n}\right\rangle \subset H^{*}\left(\mathcal{Z}_{\hat{\mathcal{K}}}\right)$. Suppose that $\psi: \hat{\mathcal{K}} \rightarrow \mathcal{K}$ is a series of edge stretchings. Then there is a non-trivial n-Massey product in $H^{*}\left(\mathcal{Z}_{\mathcal{K}}\right)$.

Proof. Since $\psi: \hat{\mathcal{K}} \rightarrow \mathcal{K}$ is a series of edge stretchings, there is a series of edge contractions $\varphi: \mathcal{K} \rightarrow \hat{\mathcal{K}}$. Given $\left\langle\hat{\alpha}_{1}, \ldots, \hat{\alpha}_{n}\right\rangle$ in $H^{*}\left(\mathcal{Z}_{\hat{\mathcal{K}}}\right)$, there is a non-trivial $n$-Massey product $\left\langle\alpha_{1}, \ldots, \alpha_{n}\right\rangle \subset H^{*}\left(\mathcal{Z}_{\mathcal{K}}\right)$ by Theorem 4.12 .

We may use edge stretchings to build infinite families of examples of Massey products in moment-angle complexes given any known Massey product in a moment-angle complex. For example we can start with one of the obstruction graphs for lowest-degree triple Massey products $[12,15]$ and produce infinite families of simplicial complexes that contain non-trivial triple Massey products of classes on different degrees. This illustrates that non-trivial Massey products are very common in moment-angle complexes, contrary to previous belief.

\section{Non-trivial Massey products in nestohedra}

Theorems 3.17 and 4.12 can be applied together to construct non-trivial higher Massey products of classes in various degrees in the cohomology of moment-angle complexes. Recall that for any simple polytope $P$, there is a simplicial complex $\mathcal{K}_{P}=\partial\left(P^{*}\right)$ and $\mathcal{Z}_{P}=\mathcal{Z}_{\mathcal{K}_{P}}$ is a moment-angle manifold. In this section we show that there are families of polytopes $P$ for which $H^{*}\left(\mathcal{Z}_{P}\right)$ has non-trivial higher Massey products. 
Nestohedra are a large family of simple polytopes built out of Minkowski sums of simplices, introduced by Feichtner and Sturmfels [14]. They include all simplices, permutahedra, Stasheff's associahedra and more generally Carr and Devadoss' graph associahedra [11]. Alternatively nestohedra are interpreted as hypergraph polytopes [13]. The first examples of Massey products in momentangle manifolds associated to nestohedra were in [19, Proposition 4.1] and [20, Lemma 4.9] and were triple Massey products constructed either by explicit calculation or using the classification of lowest degree Massey products $[12,15]$. We will use Theorems 3.17 and 4.12 to construct families of new non-trivial higher Massey products in moment-angle manifolds associated to certain nestohedra. We use a construction of nestohedra due to Postnikov [24, Theorem 7.4].

Definition 5.1. A building set $B$ is a collection of non-empty subsets of $[n+1]$ such that

1. $\{i\} \in B$ for every $i \in[n+1]$,

2. $S_{1} \cup S_{2} \in B$ for any $S_{1}, S_{2} \in B$ with $S_{1} \cap S_{2} \neq \emptyset$.

A convex polytope is the convex hull of a finite number of points in $\mathbb{R}^{n}$. If $M_{1}$ and $M_{2}$ are convex polytopes in $\mathbb{R}^{n}$, then the Minkowski sum

$$
M_{1}+M_{2}=\left\{x \in \mathbb{R}^{n} \mid x=x_{1}+x_{2}, x_{1} \in M_{1}, x_{2} \in M_{2}\right\} .
$$

is also a convex polytope.

Definition 5.2. For a building set $B \subset[n+1]$, a nestohedron $P_{B}$ is the polytope $\sum_{S \in B} \Delta^{S}$, where $\Delta^{S}=\operatorname{conv}\left\{e_{i}, i \in S\right\}$ is the convex hull of the basis elements $e_{i} \in \mathbb{R}^{n+1}$.

For example, the $n$-simplex is a nestohedron with building set $\{\{1\}, \ldots,\{n+$ $1\},[n+1]\}$. Other key examples of nestohedra are graph associahedra $P_{B_{\Gamma}}$, which are associated to a graph $\Gamma$ on the vertex set $[n+1]$. The graphical building set $B_{\Gamma}$ consists of subsets $S \subset[n+1]$ such that the restriction of $\Gamma$ to the vertices in $S$ is a connected graph.

Since every nestohedron $P_{B}$ is simple $[14,24]$, we will consider the corresponding simplicial complex $\mathcal{K}_{P_{B}}=\partial\left(P_{B}^{*}\right)$, which is the boundary of the dual polytope. Let $B_{\max }$ be the set of maximal sets in $B$ with respect to inclusion.

Proposition 5.3 ([24]). The simplicial complex $\mathcal{K}_{P_{B}}$ is isomorphic to the nested set complex $\mathcal{N}(B)$, which contains a simplex $\left\{S_{1}, \ldots, S_{k}\right\} \subset B \backslash B_{\max }$ if

1. for any $S_{i}, S_{j} \in\left\{S_{1}, \ldots, S_{k}\right\}$, either $S_{i} \subset S_{j}, S_{j} \subset S_{i}$ or $S_{i} \cap S_{j}=\emptyset$,

2. for any $S_{i_{1}}, \ldots, S_{i_{p}} \in\left\{S_{1}, \ldots, S_{k}\right\}$ with $S_{i_{j}} \cap S_{i_{l}}=\emptyset, S_{i_{1}} \sqcup \cdots \sqcup S_{i_{p}} \notin B$.

For example if $P_{B}$ is the polytopal $n$-simplex, then $\mathcal{K}_{P_{B}}$ is the boundary of an $n$-simplex. Another example is shown in Figure 16. We denote the momentangle complex $\mathcal{Z}_{\mathcal{K}_{P_{B}}}$ by $\mathcal{Z}_{P_{B}}$. 


\subsection{Permutahedra}

A permutahedron is an example of a graph associahedron, when the associated graph is a complete graph on $n+1$ vertices. Limonchenko [18, Theorem 3] showed that the 3 -dimensional permutahedron $P$ has no non-trivial triple Massey product $\left\langle\alpha_{1}, \alpha_{2}, \alpha_{3}\right\rangle$ for three-dimensional classes $\alpha_{i} \in H^{3}\left(\mathcal{Z}_{P}\right)$, using the classification by [12, Theorem 6.1.1] and [15]. However, there are other nontrivial triple Massey products in $H^{*}\left(\mathcal{Z}_{P}\right)$, as illustrated in Example 4.13. Via an explicit example, it was also shown in [19, Proposition 4.1] and [20, Lemma 4.9] that there are triple Massey products of three-dimensional classes in $H^{*}\left(\mathcal{Z}_{P}\right)$ for $n$ dimensional permutahedra $P$ with $n>3$. Here we will generalise this and show that $\mathcal{Z}_{P}$, for the $n$-dimensional permutahedron $P$, has a non-trivial $k$-Massey product for $k \leqslant n$.

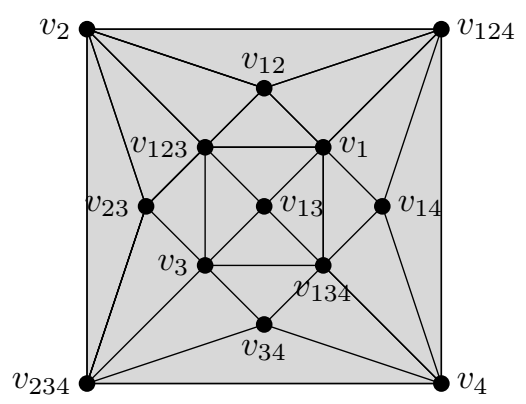

Figure 16: The simplicial complex $\mathcal{K}_{P}$, without the vertex $v_{24}$, when $P$ is the 3 -dimensional permutahedron

Proposition 5.4. When $P$ is the $n$-dimensional permutahedron, $H^{*}\left(\mathcal{Z}_{P}\right)$ has a non-trivial $k$-Massey product for every $k \leqslant n$.

Proof. The building set $B$ of the $n$-dimensional permutahedron $P$ contains all possible subsets of $[n+1]$. Let $v_{S}$ be the vertex in $\mathcal{K}_{P_{B}}$ corresponding to a set $S \in B \backslash[n+1]$. By Proposition 5.3, $\left\{v_{S_{1}}, \ldots, v_{S_{k}}\right\}$ is a simplex in $\mathcal{K}_{P_{B}}$ if for any $S_{i}, S_{j} \in\left\{S_{1}, \ldots, S_{k}\right\}$, either $S_{i} \subset S_{j}, S_{j} \subset S_{i}$. From now on we denote $\mathcal{K}_{P_{B}}$ by $\mathcal{K}$. We construct a $k$-Massey product $\left\langle\alpha_{1}, \ldots, \alpha_{k}\right\rangle \subset H^{*}\left(\mathcal{Z}_{\mathcal{K}}\right)$ by explicitly defining $J_{i}$ and $\alpha_{i} \in \widetilde{H}^{0}\left(\mathcal{K}_{J_{i}}\right)$. Then we edge contract $\mathcal{K}_{J_{1} \cup \ldots \cup J_{k}}$ to a simplicial complex that by Construction 3.5 has a non-trivial Massey product. For $k<n$, let

$$
\begin{aligned}
& \alpha_{1} \in \widetilde{H}^{0}\left(\mathcal{K}_{v_{\{1\}}, v_{\{2\}}}\right) \\
& \alpha_{i} \in \widetilde{H}^{0}\left(\mathcal{K}_{v_{\{1, \ldots, i, k+1\}}, v_{\{2, \ldots, i+1\}}}\right) \text { for } 1<i<k \\
& \alpha_{k} \in \widetilde{H}^{0}\left(\mathcal{K}_{v_{\{1, \ldots, k+1\}}, v_{\{1, \ldots, k, k+2\}}}\right)
\end{aligned}
$$

so $\alpha_{i}$ corresponds to a class $\alpha_{i} \in H^{3}\left(\mathcal{Z}_{\mathcal{K}}\right)$. In this case $\left|J_{i}\right|=2$, so there are no edges to contract. Let $\hat{\mathcal{K}}=\mathcal{K}_{J_{1} \cup \ldots \cup J_{k}}$. There is no edge $\left\{v_{\{1\}}, v_{\{2, \ldots, i+1\}}\right\}$ in $\hat{\mathcal{K}}$ for $v_{\{1\}} \in J_{1}$ and $v_{\{2, \ldots, i+1\}} \in J_{i}$ since $\{1\} \not \subset\{2, \ldots, i+1\}$. Also there is no edge 
$\left\{v_{\{1, \ldots, i, k+1\}}, v_{\{2, \ldots, j+1\}}\right\}$ nor $\left\{v_{\{1, \ldots, i, k+1\}}, v_{\{1, \ldots, k, k+2\}}\right\}$ for $v_{\{1, \ldots, i, k+1\}} \in J_{i}$, $v_{\{2, \ldots, j+1\}} \in J_{j}$ with $1<i<j<k$ and $v_{\{1, \ldots, k, k+2\}} \in J_{k}$. All the other edges are in $\hat{\mathcal{K}}$. That is, $\left\{v_{\{1\}}, v_{\{1, \ldots, i, k+1\}}\right\} \in \hat{\mathcal{K}}$ and $\left\{v_{\{1\}}, v_{\{1, \ldots, k, k+2\}}\right\} \in \hat{\mathcal{K}}$ for $v_{\{1\}} \in J_{1}, v_{\{1, \ldots, i, k+1\}} \in J_{i}$ for any $i \leqslant k$ and $v_{\{1, \ldots, k, k+2\}} \in J_{k}$. Similarly, $\left\{v_{\{1, \ldots, i, k+1\}}, v_{\{1, \ldots, j, k+1\}}\right\} \in \hat{\mathcal{K}}$ for $v_{\{1, \ldots, i, k+1\}} \in J_{i}$ and $v_{\{1, \ldots, j, k+1\}} \in J_{j}$ with $1<i<j \leqslant k$. Also $\left\{v_{\{2, \ldots, i+1\}}, v_{S_{j}}\right\} \in \hat{\mathcal{K}}$ for $v_{\{2, \ldots, i+1\}} \in J_{i}$ and any $v_{S_{j}} \in$ $J_{j}$ with $1 \leqslant i<j \leqslant k$. Therefore $\hat{\mathcal{K}}$ is obtained from the join $\mathcal{K}_{J_{1}} * \cdots *$ $\mathcal{K}_{J_{k}}$ by star deleting at the edges $\left\{v_{\{1\}}, v_{\{2, \ldots, i+1\}}\right\},\left\{v_{\{1, \ldots, i, k+1\}}, v_{\{2, \ldots, j+1\}}\right\}$ and $\left\{v_{\{1, \ldots, i, k+1\}}, v_{\{1, \ldots, k, k+2\}}\right\}$ for $v_{\{1\}} \in J_{1}, v_{\{2, \ldots, i+1\}} \in J_{i}, v_{\{1, \ldots, i, k+1\}} \in$ $J_{i}, v_{\{2, \ldots, j+1\}} \in J_{j}$ with $1<i<j<k$ and $v_{\{1, \ldots, k, k+2\}} \in J_{k}$. Hence by Theorem 3.17, the Massey product $\left\langle\alpha_{1}, \ldots, \alpha_{k}\right\rangle \subset H^{2 k+2}\left(\mathcal{Z}_{\mathcal{K}}\right)$ is non-trivial.

For $k=n$, let

$$
\begin{aligned}
& \alpha_{1} \in \widetilde{H}^{0}\left(\mathcal{K}_{v_{\{1\}}, v_{\{2, \ldots, n+1\}}, v_{\{3, \ldots, n+1\}}}\right) \\
& \alpha_{i} \in \widetilde{H}^{0}\left(\mathcal{K}_{v_{\{1, \ldots, i\}}, v_{\{2, \ldots, i\}}, v_{\{3, \ldots, i+1\}}}\right) \text { for } 1<i<n \\
& \alpha_{n} \in \widetilde{H}^{0}\left(\mathcal{K}_{\left.v_{\{1, \ldots, n\}}, v_{\{2, \ldots, n\}}, v_{\{1,3, \ldots, n+1\}}\right) .}\right.
\end{aligned}
$$

Since $\left|J_{i}\right|=3$ for every $i \in\{1, \ldots, n\}$, we will perform $n$ edge contractions in order to obtain a simplicial complex $\hat{\mathcal{K}}$ on $2 n$ vertices. There is an edge $\left\{v_{\{2, \ldots, n+1\}}, v_{\{3, \ldots, n+1\}}\right\} \in \mathcal{K}_{J_{1}}$ since $\{3, \ldots, n+1\} \subset\{2, \ldots, n+1\}$. Also there are edges $\left\{v_{\{1, \ldots, i\}}, v_{\{2, \ldots, i\}}\right\} \in \mathcal{K}_{J_{i}}$ for $1<i \leqslant n$. Since $P$ is a simple polytope, $\mathcal{K}$ is a triangulation of a sphere so the contraction of these edges satisfy the link condition. Let $\hat{\mathcal{K}}$ be obtained from $\mathcal{K}_{J_{1} \cup \ldots \cup J_{n}}$ by contracting these $n$ edges. Then as in the case when $k<n, \hat{\mathcal{K}}$ is a simplicial complex obtained from the join of $n$ pairs of disjoint vertices by star deletions as described by Construction 3.17. Hence by Theorem 3.17, there is a non-trivial $k$-Massey product in $H^{*}\left(\mathcal{Z}_{\hat{\mathcal{K}}}\right)$. By Theorem 4.12 , the Massey product $\left\langle\alpha_{1}, \ldots, \alpha_{k}\right\rangle \subset H^{*}\left(\mathcal{Z}_{\mathcal{K}}\right)$ is also nontrivial.

A similar technique to that used in Proposition 5.4 can be applied to other simple polytopes. An example is the family of stellohedra: graph associahedra corresponding to star graphs, which are graphs with a central vertex and edges attaching every other vertex to the central one. It was shown in [18, Theorem 3] that there are 3 -Massey products on 3 -dimensional classes in $H^{*}\left(\mathcal{Z}_{P}\right)$ when $P$ is a 3 -dimensional stellohedron, using the classification in $[12,15]$. By applying Theorems 3.17 and 4.12 , we generalise that result by constructing non-trivial $n$-Massey products in moment-angle manifolds over $n$-dimensional stellohedron.

Proposition 5.5. When $P$ is the $n$-dimensional stellohedron, $H^{*}\left(\mathcal{Z}_{P}\right)$ has a non-trivial $n$-Massey product.

Proof. As in Proposition 5.4, we construct $\alpha_{i} \in \widetilde{H}^{0}\left(\mathcal{K}_{J_{i}}\right)$ where $\mathcal{K}=\mathcal{K}_{P}$. Let the star graph associated to $P$ be labelled so that the central vertex is 1 and the other vertices are $2, \ldots, n+1$. The building set for $P$ is

$$
\{\{1\}, \ldots,\{n+1\},\{1,2\}, \ldots,\{1, n+1\}, \ldots,\{1, \ldots, n\},\{1, \ldots, n-1, n+1\},[n+1]\} .
$$


Let

$$
\begin{aligned}
& \alpha_{1} \in \widetilde{H}^{0}\left(\mathcal{K}_{v_{\{2\}}, v_{\{1\}}}\right) \\
& \alpha_{i} \in \widetilde{H}^{0}\left(\mathcal{K}_{v_{\{1, \ldots, i\}}, v_{\{1,3, \ldots, i+2\}}, v_{\{1,4, \ldots, i+2\}}}\right) \text { for } 1<i<n \\
& \alpha_{n} \in \widetilde{H}^{0}\left(\mathcal{K}_{\left.v_{\{1,3\}}, v_{\{3\}}, v_{\{1,2,4, \ldots, n+1\}}\right) .}\right.
\end{aligned}
$$

By contracting the edges $\left\{v_{\{1,3, \ldots, i+2\}}, v_{\{1,4, \ldots, i+2\}}\right\} \in \mathcal{K}_{J_{i}}$ for $1<i<n$ and the edge $\left\{v_{\{1,3\}}, v_{\{3\}}\right\} \in \mathcal{K}_{J_{n}}$, we obtain a simplicial complex $\hat{\mathcal{K}}$ that is constructed from the join of $n$ disjoint points by star deletions as in Construction 3.5.

Propositions 5.4 and 5.5 reiterate that the moment-angle manifolds associated to permutahedra and stellohedra are non-formal [20]. Also, the families of permutahedra and stellohedra are examples of geometric direct families of polytopes, whose moment-angle manifolds are studied in [9]. Hence, Propositions 5.4 and 5.5 answer Problems 5.32, 5.34 and 5.35 in [9], which ask if there are geometric direct families of polytopes with non-trivial higher Massey products.

\subsection{Non-trivial indeterminacy and permutahedra}

Massey products with non-trivial indeterminacy can be found in momentangle manifolds. We illustrate this in moment-angle manifolds associated with permutahedra. We first construct an example of a 4-Massey product with nontrivial indeterminacy in a moment-angle complex using Theorem 3.23, then find a full-subcomplex of a permutahedron that edge contracts to this example and apply Theorem 4.12 .

Example 5.6. Let $\mathcal{K}^{i}$ be a pair of disjoint points $J_{i}=\left\{i, i^{\prime}\right\}$ for $i=1, \ldots, 4$ and define

$$
\mathcal{K}=\operatorname{sd}_{\left\{1,2^{\prime}\right\}} \operatorname{sd}_{\left\{1,3^{\prime}\right\}} \operatorname{sd}_{\left\{2,3^{\prime}\right\}} \operatorname{sd}_{\left\{2,4^{\prime}\right\}} \operatorname{sd}_{\left\{3,4^{\prime}\right\}} \operatorname{sd}_{\left\{1^{\prime}, 2^{\prime}\right\}} \operatorname{sd}_{\left\{1^{\prime}, 3^{\prime}\right\}} \mathcal{K}^{1} * \mathcal{K}^{2} * \mathcal{K}^{3} * \mathcal{K}^{4} .
$$

Let $\alpha_{i}=\left[a_{i}\right]$ and $a_{i}=\chi_{i} \in C^{0}\left(\mathcal{K}_{J_{i}}\right)$. By Theorem 3.23, $\left\langle\alpha_{1}, \alpha_{2}, \alpha_{3}, \alpha_{4}\right\rangle \subset$ $H^{*}\left(\mathcal{Z}_{\mathcal{K}}\right)$ is non-trivial with non-trivial indeterminacy.

Proposition 5.7. There are non-trivial Massey products with non-trivial indeterminacy in moment-angle manifolds corresponding to permutahedra.

Proof. Let $P$ be the 5 -dimensional permutahedron. Denote $\mathcal{K}_{\mathcal{P}}$ by $\mathcal{K}$. Recall that by Proposition 5.3, $\left\{v_{S_{1}}, \ldots, v_{S_{k}}\right\}$ is a simplex in $\mathcal{K}$ if for any $S_{i}, S_{j} \in$ $\left\{S_{1}, \ldots, S_{k}\right\}$, either $S_{i} \subset S_{j}$ or $S_{j} \subset S_{i}$. Let

$$
\begin{aligned}
& J_{1}=\left\{v_{\{1\}}, v_{\{2\}}, v_{\{2,5\}}, v_{\{5\}}\right\} \\
& J_{2}=\left\{v_{\{1,2\}}, v_{\{3\}}\right\} \\
& J_{3}=\left\{v_{\{1,2,3\}}, v_{\{2,3\}}, v_{\{3,4\}}\right\} \\
& J_{4}=\left\{v_{\{1,2,3,4\}}, v_{\{2,3,4\}}, v_{\{1,3,4,5\}}\right\}
\end{aligned}
$$


and let $\alpha_{i} \in \widetilde{H}^{0}\left(\mathcal{K}_{J_{i}}\right)$. Let $\hat{\mathcal{K}}$ be the simplicial complex in Example 5.6, so there is a non-trivial 4-Massey product in $H^{*}\left(\mathcal{Z}_{\hat{\mathcal{K}}}\right)$. Consider the map $\varphi: \mathcal{K} \rightarrow \hat{\mathcal{K}}$ that takes $J_{i} \mapsto\left\{i, i^{\prime}\right\}$ by contracting the edges

$$
\begin{aligned}
\left\{v_{\{2\}}, v_{\{2,5\}}\right\},\left\{v_{\{2,5\}}, v_{\{5\}}\right\} & \mapsto 1^{\prime} \\
\left\{v_{\{1,2,3\}}, v_{\{2,3\}}\right\} & \mapsto 3 \\
\left\{v_{\{1,2,3,4\}}, v_{\{2,3,4\}}\right\} & \mapsto 4
\end{aligned}
$$

Since $\mathcal{K}$ is a triangulation of a sphere, these edge contractions satisfy the link condition. Therefore by Theorem 4.12, there is a non-trivial 4-Massey product $\left\langle\alpha_{1}, \alpha_{2}, \alpha_{3}, \alpha_{4}\right\rangle \subset H^{*}\left(\mathcal{Z}_{\mathcal{K}}\right)$ for $\alpha_{i} \in H^{0}\left(\mathcal{K}_{i}\right)$, and this 4-Massey product has non-trivial indeterminacy.

This example of a non-trivial $n$-Massey product with non-trivial indeterminacy can be reproduced in any $(n+1)$-dimensional permutahedron.

\section{References}

[1] D. Attali, A. Lieutier, and D. Salinas. Efficient data structure for representing and simplifying simplicial complexes in high dimensions. Internat. J. Comput. Geom. Appl., 22(4):279-303, 2012.

[2] A. Bahri, M. Bendersky, F. R. Cohen, and S. Gitler. Cup-products for the polyhedral product functor. Math. Proc. Cambridge Philos. Soc., 153(3):457-469, 2012.

[3] D. Baralić, J. Grbić, I. Y. Limonchenko, and A. Vučić. Toric objects associated with the dodecahedron. Filomat, 34(7):2329-2356, 2020.

[4] I. V. Baskakov. Cohomology of $K$-powers of spaces and the combinatorics of simplicial divisions. Uspekhi Mat. Nauk, 57(5(347)):147-148, 2002.

[5] I. V. Baskakov. Triple Massey products in the cohomology of moment-angle complexes. Uspekhi Mat. Nauk, 58(5(353)):199-200, 2003.

[6] F. Bosio and L. Meersseman. Real quadrics in $\mathbf{C}^{n}$, complex manifolds and convex polytopes. Acta Math., 197(1):53-127, 2006.

[7] V. M. Buchstaber and T. E. Panov. Torus actions, combinatorial topology, and homological algebra. Russ. Math. Surv., 55(5):825-921, 2000.

[8] V. M. Bukhshtaber, N. Y. Erokhovets, M. Masuda, T. E. Panov, and S. Park. Cohomological rigidity of manifolds defined by 3-dimensional polytopes. Uspekhi Mat. Nauk, 72(2(434)):3-66, 2017.

[9] V. M. Bukhshtaber and I. Y. Limonchenko. Massey products, toric topology and the combinatorics of polytopes. Izv. Ross. Akad. Nauk Ser. Mat., 83(6):3-62, 2019. 
[10] V. M. Bukhshtaber and T. E. Panov. Torus actions and the combinatorics of polytopes. Tr. Mat. Inst. Steklova, 225(Solitony Geom. Topol. na Perekrest.):96-131, 1999.

[11] M. Carr and S. Devadoss. Coxeter complexes and graph-associahedra. Topology and its Applications, 153:2155-2168, 062006.

[12] G. Denham and A. I. Suciu. Moment-angle complexes, monomial ideals and Massey products. Pure Appl. Math. Q., 3(1, Special Issue: In honor of Robert D. MacPherson. Part 3):25-60, 2007.

[13] K. Došen and Z. Petrić. Hypergraph polytopes. Topology and its Applications, 158(12):1405 - 1444, 2011. Special Issue: Analysis, Topology and Applications 2010 (ATA 2010).

[14] E. M. Feichtner and B. Sturmfels. Matroid polytopes, nested sets and bergman fans. Portugaliae Mathematica. Nova Série, 62(4):437-468, 2005.

[15] J. Grbić and A. Linton. Lowest-degree triple Massey products in momentangle complexes. Uspekhi Mat. Nauk, 75(6(456)):175-176, 2020.

[16] M. Hochster. Cohen-Macaulay rings, combinatorics, and simplicial complexes. Lecture Notes in Pure and Appl. Math., 26:171-223, 1977.

[17] D. Kraines. Massey higher products. Trans. Amer. Math. Soc., 124:431449, 1966.

[18] I. Limonchenko. Massey products in the cohomology of moment-angle manifolds of 2-truncated cubes. Uspekhi Mat. Nauk, 71(2(428)):207-208, 2016.

[19] I. Limonchenko. Topology of moment-angle manifolds arising from flag nestohedra. Chin. Ann. Math. Ser. B, 38(6):1287-1302, 2017.

[20] I. Y. Limonchenko. On higher Massey products and rational formality for moment-angle manifolds over multiwedges. Tr. Mat. Inst. Steklova, 305(Algebraicheskaya Topologiya Kombinatorika i Matematicheskaya Fizika):174$196,2019$.

[21] W. S. Massey. A new cohomology invariant of topological spaces. Bull. Amer. Math. Soc., 57(74), 1951.

[22] J. P. May. Matric Massey products. J. Algebra, 12:533-568, 1969.

[23] T. Panov and Y. Ustinovsky. Complex-analytic structures on moment-angle manifolds. Mosc. Math. J., 12(1):149-172, 216, 2012.

[24] A. Postnikov. Permutohedra, associahedra, and beyond. Int. Math. Res. Not. IMRN, (6):1026-1106, 2009.

[25] H. Uehara and W. S. Massey. The Jacobi identity for Whitehead products. In Algebraic geometry and topology. A symposium in honor of S. Lefschetz, pages 361-377. Princeton University Press, Princeton, N. J., 1957. 
[26] E. G. Zhuravleva. Massey products in the cohomology of the momentangle manifolds corresponding to pogorelov polytopes. Mathematical Notes, 105(3):519-527, 2019. 